\title{
Musk Compounds in the Nordic environment
}





\section{Musk Compounds in the Nordic environment}

Betty Bügel Mogensen, Gunnar Pritzl, Suresh Rastogi, Ola Glesne, Britta Hedlund, Juha-Pekka Hirvi, Alf Lundgren \& Albert Sigurdsson

TemaNord 2004:503 


\section{Musk Compounds in the Nordic environment}

TemaNord 2004:503

(C) Nordic Council of Ministers, Copenhagen 2004

ISBN 92-893-0981-4

ISSN 0908-6692

Print: Ekspressen Tryk \& Kopicenter

Copies: 400

Printed on paper approved by the Nordic Environmental Labelling.

This publication may be purchased from any of the sales agents listed on the last page.

Nordic Council of Ministers

Store Strandstræde 18

DK-1255 Copenhagen K

Phone (+45) 33960200

Fax (+45) 33960202

\section{Nordic Council}

Store Strandstræde 18

DK-1255 Copenhagen K

Phone (+45) 33960400

Fax $\quad(+45) 33111870$

www.norden.org

\section{Nordic Environmental Co-operation}

Environmental co-operation is aimed at contributing to the improvement of the environment and forestall problems in the Nordic countries as well as on the international scene. The cooperation is conducted by the Nordic Committee of Senior Officials for Environmental Affairs. The co-operation endeavours to advance joint aims for Action Plans and joint projects, exchange of information and assistance, e.g. to Eastern Europe, through the Nordic Environmental Finance Corporation (NEFCO).

\section{The Nordic Council of Ministers}

was established in 1971. It submits proposals on co-operation between the governments of the five Nordic countries to the Nordic Council, implements the Council's recommendations and reports on results, while directing the work carried out in the targeted areas. The Prime Ministers of the five Nordic countries assume overall responsibility for the co-operation measures, which are co-ordinated by the ministers for co-operation and the Nordic Co-operation committee. The composition of the Council of Ministers varies, depending on the nature of the issue to be treated.

\section{The Nordic Council}

was formed in 1952 to promote co-operation between the parliaments and governments of Denmark, Iceland, Norway and Sweden. Finland joined in 1955. At the sessions held by the Council, representatives from the Faroe Islands and Greenland form part of the Danish delegation, while Åland is represented on the Finnish delegation. The Council consists of 87 elected members - all of whom are members of parliament. The Nordic Council takes initiatives, acts in a consultative capacity and monitors co-operation measures. The Council operates via its institutions: the Plenary Assembly, the Presidium and standing committees. 


\section{Contents}

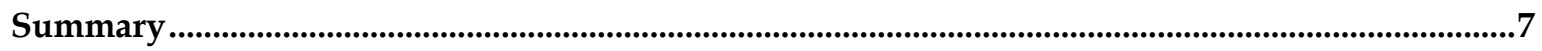

Resumé 9

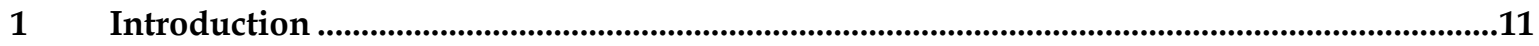

2 Background ...................................................................................................................................13

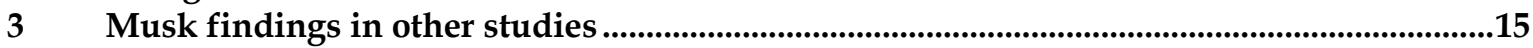

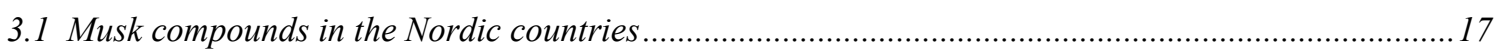

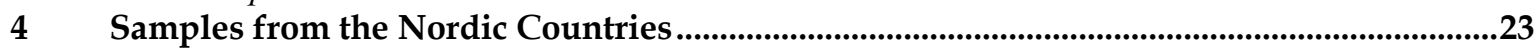

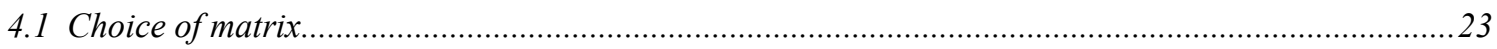

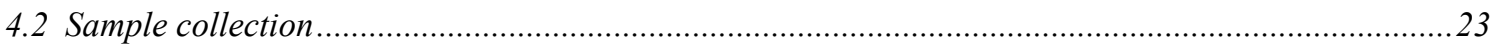

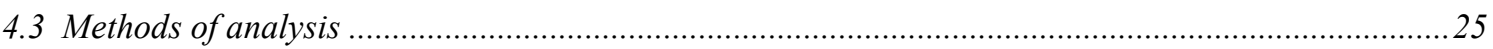

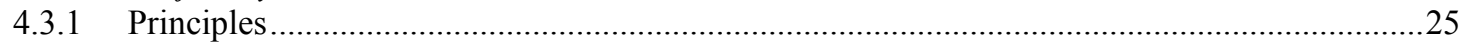

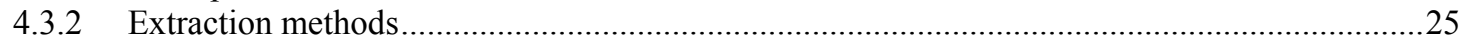

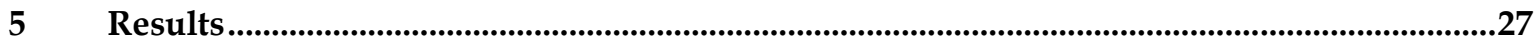

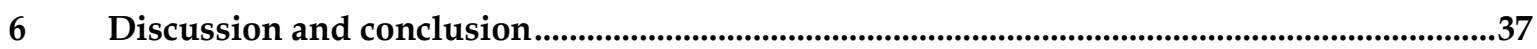

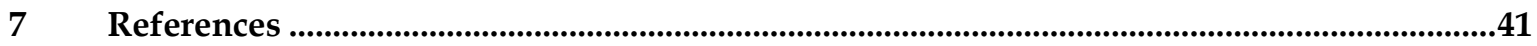

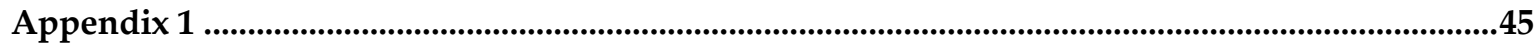

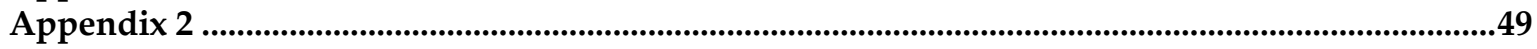

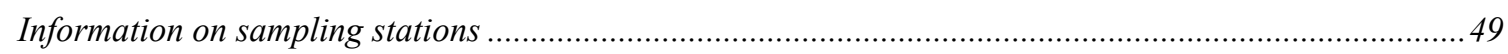

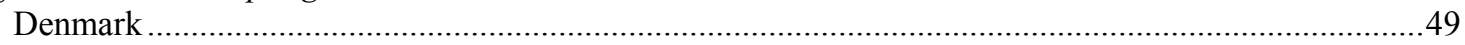

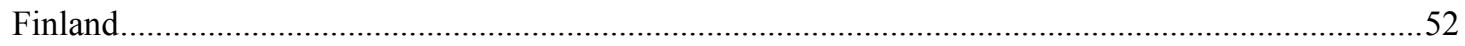

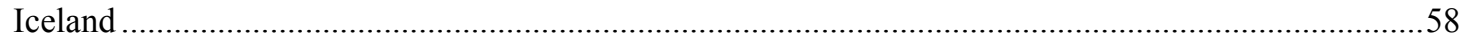

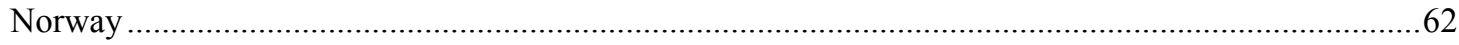

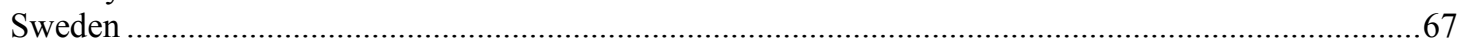




\section{Summary}

A screening project of synthetic musk compounds in the environment of the Nordic countries was carried out in the second half of 2002. The project was initiated by the Nordic Terrestrial Monitoring Group, NTEM, with support from the Nordic Council of Ministers and from each of the Nordic Countries (Monitoring and Data Group and Chemicals group).

The project was carried out by the National Environmental Research Institute (NERI) in Denmark with the NTEM group serving as a steering group.

Each country was responsible for collection and transport of the samples according to a sampling manual.

23 samples of rain water, 27 samples of sludge from sewage treatment plants, 20 samples of blue mussels and 15 samples of liver from red fox, polar fox or racoon dog have been analysed for content of nine synthetic musk compounds i.e. six polycyclic musks: Cashmeran (DPMI), Celestolide (ADBI), Phantolide (AHDI), Traseolide (ATII), Galaxolide (HHCB) and Tonalide (AHTN) and three nitro-musk compounds: Musk xylene, musk keton and musk ambrette. These compounds represent the major part of synthetic musk compounds used in cosmetics, washing and cleaning agents etc. The results show high concentrations of especially polycyclic musk compounds in sewage sludge, maximum concentration of Galaxolide being $26,500 \mu \mathrm{g} / \mathrm{kg}$ dry weight and of Tonalide $3,600 \mu \mathrm{g} / \mathrm{kg}$ dry weight. Polycyclic musks were detected in 11/20 samples of blue mussels. Celestolide is detected most frequently and at the highest concentration, $11,500 \mathrm{ng} / \mathrm{g}$ lipid.

Galaxolide was detected in 4/23 rainwater samples at concentrations in the range 12-29 ng/l. One sample additionally contained tonalide $35 \mathrm{ng} / \mathrm{l}$.

Nitro musk compounds were only exceptionally detected in any samples

The environmental significance of the findings is difficult to assess, as there is a lack of data concerning effects of synthetic musk compounds.

To reduce the burden of the nordic environments with musk compounds following steps may be required: Specific regulations concerning emissions, updating sewage plants to efficiently degrade the musk compounds, and monitoring of musk compound in the environment, effluents and sewage sludge in particular. Monitoring of musk compounds in aquatic fauna is also relevant and should be focused on tissue with high lipid content. In rain water galaxolide and tonalide could serve as indicator compounds in monitoring programmes. 


\section{Resumé}

Et screeningsprojekt der omfatter syntetiske musk stoffer i miljøet i de nordiske lande, blev gennemført $i$ anden halvdel af 2002. Projektet blev igangsat af gruppen Nordic Terrestrial Environmental Monitoring group, NTEM, med støtte fra Nordisk Ministerråd, Monitering og Data gruppen og Kemikaliegruppen.

Projektet blev udført af Danmarks Miljøundersøgelser (DMU) idet NTEM gruppen fungerede som styringsgruppe.

Hvert land havde ansvar for indsamling og forsendelse af prøverne i henhold til en rundsendt manual.

23 regnvandsprøver, 27 prøver af spildevandsslam, 20 prøver af blåmuslinger og 15 prøver af lever fra rød ræv, polarræv eller mårhund blev analyseret for indhold af 9 syntetiske musk stoffer. Disse består af 6 polycykliske musk stoffer: Cashmeran (DPMI), Celestolide (ADBI), Phantolide (AHDI), Traseolide (ATII), Galaxolide (HHCB) og Tonalide (AHTN) og tre nitro-musk forbindelser: Musk xylene, musk keton og musk ambrette. Disse stoffer repræsenterer størstedelen af de syntetiske musk stoffer, der finder anvendelse i kosmetik, rengørings- og rensemidler m.m.

Resultaterne viser høje koncentrationer af især de polycykliske musk stoffer I spildevandsslam. Koncentrationen af Galaxolide er op til $26.000 \mu \mathrm{g} / \mathrm{kg}$ tørstof og af Tonalide op til $3.600 \mu \mathrm{g} / \mathrm{kg}$ tørstof.

Polycykliske musk stoffer blev påvist i 11/20 prøver af blåmuslinger. Celestolide er det stof, der oftest er påvist og det findes i den højeste koncentration, $11.500 \mathrm{ng} / \mathrm{g}$ fedtstof. Galaxolide blev påvist i 4/23 regnvandsprøver i koncentrationen 12-29 ng/l. En prøve indeholdt desuden Tonalide i koncentrationen $35 \mathrm{ng} / \mathrm{l}$.

Nitro musk forbindelser blev kun undtagelsesvis detekteret i nogen af prøverne. Det er vanskeligt at vurdere den miljømæssige betydning af fundene af musk stoffer, idet der mangler data vedrørende effekten af syntetiske musk stoffer i miljøet.

For at nedsætte belastningen af miljøet i de nordiske lande med musk stoffer, kan det være nødvendigt at gennemføre nogle tiltag: Specifikke reguleringer af udledningen, forbedring af rensningsanlæggenes nedbrydning af musk stofferne og monitering af musk forbindelser i miljøet. Moniteringen bør især rette sig mod spildevandsslam og udløbsvand fra rensningsanlæg. Monitering af musk forbindelser i akvatisk fauna er også relevant og bør fokusere på væv med højt fedtindhold. I regnvand kan Galaxolide og Tonalide med fordel bruges som indikatorstoffer I moniteringsprogrammer. 


\section{Introduction}

The Nordic environmental collaboration (Nordiske miljøsamarbejde) $2001-2004$ is a program directed by the Nordic Council of Ministers. It is meant to be a collaborative tool to guide and prioritise the activities and initiatives that must be started. Chemicals have high priority, and the aim is to ensure that the use of chemicals does not involve the risk of negative effects on human health and the environment. Furthermore the emissions of chemicals, that present a threat to health and the environment, must cease within a generation, defined as 25 years (Det Nordiske Miljøhandlingsprogram 20012004).

To fulfil these objectives it is important to clarify and describe the state-of-the-art with respect to the occurrence of specific chemicals. The working group Nordic Terrestrial Environmental Monitoring group (NTEM) was supported by the Nordic Council of Ministers (Monitoring and Data group and Chemicals group) to carry out screening of potentially harmful chemicals in the environment. In 2001 musk compounds were selected for screening.

The NTEM group encompasses one member and one substitute from each of the Nordic Countries. The members constitute the steering group for the project. These are Ola Glesne, Norwegian Pollution Control Authority, Norway (chairman), Britta Hedlund, Swedish Environmental Protection Agency, Sweden, Juha-Pekka Hirvi, Finnish Environment Institute, Finland, Albert Sigurdsson, Environment and Food Agency, Iceland and Betty Bügel Mogensen, National Environmental Research Institute, Denmark and Alf Lundgren from National Chemicals Inspectorate in Sweden who represents The Nordic Chemicals group in the steering group. The steering group has designed the screening programme and prepared a tender invitation. The steering group has been responsible for collection and sending of all samples included in this project. The group wants to acknowledge all the national institutes, authorities and private people in the Nordic countries who have contributed to the sample collection. The analysis programme and the reporting were carried out at NERI after a tender procedure, in which Erik Kirknel from the Danish Institute of Agricultural Science represented Denmark in the steering group. This report presents the results from this screening programme and discusses the results. The authors want to thank laboratory technicians Celestin Gnahore and Claus Jacobsen for their efforts with the chemical analyses. 


\section{Background}

Synthetic musks mainly encompass two groups of compounds: Nitro musks and polycyclic musks. The nitro musks include a group of five synthetic alkylated nitro benzenes: musk moskene, musk tibetene, musk xylene, musk ketone and musk ambrette. The polycyclic musks are chemically alkylated tetralin or indane systems. Main representatives are Galaxolide (HHCB), Tonalide (AHTN), Celestolide (ADBI), Phantolide (AHMI) and Traseolide (AITI) (Herren and Berset 2000). Cashmeran (DPMI) resembles the polyclic musks and is considered to be a polycyclic musk by Rimkus 1998, but structure, physical and chemical properties and odour differ from those of regular polycyclic musks. A sixth polycyclic musk, Versalide, has not been produced since 1980 because of strong neurotoxic effects (Rimkus 1999). Galaxolide and Tonalide are the two most important polycyclic musks probably sharing $95 \%$ of the market for polycyclic musks.

The musk compounds are widely used as fragrance ingredients in washing and cleaning agents, fabric softeners, air fresheners, shampoos, perfumes and other cosmetic products, as food additives in fish baits and in cigarettes (Herren and Berset 2000, Kallenborn et al 1999). Human skin is exposed directly to musk compounds from many of these products. From the use in human household products synthetic musks are released to the atmosphere and to waste water. From wastewater treatment plants the compounds are released with effluents to the aquatic environment The EU Commission has initiated a risk assessment of musk ketone and musk xylene. The authors have had access to draft reports on the environmental risk assessments, EU 2002 and EU 2003. Musk ketone and musk xylene shows no degradation in biodegradation tests. However, measurements of influent and effluent in sewage treatment plants indicate that $80-92 \%$ of musk ketone and $95-98 \%$ of musk xylene are removed by the treatment in the plant. It is suggested that apart from adsorption also a biotransformation take place during an anaerobic phase of the treatment reducing the nitro musks into the corresponding amino compounds. Musk compounds are only partly degraded in sewage treatment plants so they are introduced to the environment via effluent from sewage plants and are present in sewage sludge. The compounds are stable in the environment and may also bioaccumulate in the food chain (SuterEichenberg et al 1998). Bioconcentration factor of musk xylene is 1,600 1/kg (EU 2003) and that of musk ketone 1380 1/kg (EU 2002) whole fish wet weight.

The import of musk ketone and musk xylene in Europe in 2000 was 35 tonnes and 67 tonnes respectively (EU 2002 and EU 2003). The two polycyclic musks Galaxolide and Tonalide were used in amounts of $3285 \mathrm{t}$ in 1992 and $2067 \mathrm{t}$ in 1995 (Kallenborn et al 1999). Consumption of nitromusks in Norway in 2001 was $400 \mathrm{~kg}$ of musk ketone and $200 \mathrm{~kg}$ of musk xylene. The estimated maximum emission of musk xylene to the environment was $0.3 \mathrm{~kg}$ to the atmosphere and $338 \mathrm{~kg}$ to the aquatic environment with cosmetics and cleaning agents as primary source. (Glesne 2003). Consumption in Denmark according to the Danish Product Register is: Galaxolide 333 $\mathrm{kg} /$ year mainly used in cosmetics and cleaning agents, Tonalide $33 \mathrm{~kg} /$ year and Traseolide $14 \mathrm{~kg} /$ year both mainly in cleaning agents, Cashmeran and Celestolide about $1 \mathrm{~kg} /$ year and Phantolide less than $1 \mathrm{~kg} /$ year. Musk xylene, $134 \mathrm{~kg} /$ year and musk ketone, $20 \mathrm{~kg} /$ year are mainly used in cosmetics. Information about use of fragrances 
forwarded to the Product Register is given voluntarily. The comsumption indicated above is therefore a minimum value.

Musk compounds have been identified in the aquatic environment and in human milk. However, prior to this project only little was known about occurrence of musk compounds in the environment in the Nordic Countries. Because of the lack of Nordic data, the high production volume and the environmental properties mentioned above, the NTEM working group selected musk compounds as the first group of pollutants to be screened in a joint Nordic programme. The EU Commission's Scientific Committee on Cosmetic Products and Non-Food Products intended for Consumers (SCCNFP) has given opinions on safety evaluation of use of some musk fragrances in consumer products. However, the committee has not considered possible effects in the environment.

Table 3.1 provides an overview of names and chemical structures of the musk compounds that are included in the current project. Table displays physical chemical data for some of the compounds. 


\section{Musk findings in other studies}

A full review of musk compounds in the environment was not intended in this report. However, this chapter provides an overview of some recent findings. Only musk compounds that are analysed in the current screening project are referred. These compounds are listed in Table 3.1

Table 3.1 Common name/trade name, chemical name, CAS no. and chemical structure of musk compounds included in the analysis programme

\begin{tabular}{|c|c|c|c|}
\hline $\begin{array}{l}\text { Common name/trade } \\
\text { name }\end{array}$ & $\begin{array}{l}\text { Chemical name } \\
\text { (Short name) }\end{array}$ & CAS no. & Structure \\
\hline Cashmeran & $\begin{array}{l}\text { 6,7-Dihydro-1,1,2,3,3- } \\
\text { pentamethyl-4(5H)indanone } \\
\text { (DPMI) }\end{array}$ & $33704-61-9$ & \\
\hline Celestolide & $\begin{array}{l}\text { 4-Acetytl-1,1-dimethyl-6-tert- } \\
\text { butyldihydroindene (ADBI) }\end{array}$ & 13171-00-1 & \\
\hline Phantolide & $\begin{array}{l}\text { 6-Acetyl-1,1,2,3,3,5- } \\
\text { hexamethyldihydroindene } \\
\text { (AHDI) }\end{array}$ & $15323-35-0$ & \\
\hline Musk ambrette & $\begin{array}{l}\text { 1-tert-Butyl-2-methoxy-4- } \\
\text { methyl-3,5-dinitrobenzene (MA) }\end{array}$ & $83-66-9$ & \\
\hline Traseolide & $\begin{array}{l}\text { 5-Acetyl-1,1,2,6-tetramethyl-3- } \\
\text { isopropyl-dihydroindene (ATII) }\end{array}$ & $68140-48-7$ & \\
\hline
\end{tabular}




\begin{tabular}{|c|c|c|c|}
\hline $\begin{array}{l}\text { Common name/trade } \\
\text { name }\end{array}$ & $\begin{array}{l}\text { Chemical name } \\
\text { (Short name) }\end{array}$ & CAS no. & Structure \\
\hline Musk xylene & $\begin{array}{l}\text { 1-tert-Butyl-3,5-dimethyl-2,4,6- } \\
\text { trinitrobenzene (MX) }\end{array}$ & $81-15-2$ & \\
\hline Galaxolide & $\begin{array}{l}\text { 1,3,4,6,7,8-Hexahydro- } \\
4,6,6,7,8,8 \text {-hexamethyl- } \\
\text { cyclopenta[g]-2-benzopyrane } \\
\text { (HHCB) }\end{array}$ & $1222-05-5$ & \\
\hline Tonalide & $\begin{array}{l}\text { 7-Acetyl-1,1,3,4,4,6- } \\
\text { hexamethyltetrahydro-naphtlene } \\
\text { (AHTN) }\end{array}$ & $1506-02-1$ & \\
\hline Musk ketone & $\begin{array}{l}\text { 1-tert-Butyl-3,5-dimethyl-2,6- } \\
\text { dinitrobenzene (MK) }\end{array}$ & $81-14-1$ & \\
\hline
\end{tabular}

Physical chemical data for most of the compounds were available from Nylander 2001. (Table 3.2) Musk xylene and musk ketones are similar in properties and musk ambrette will possibly have similar properties as well. Cashmeran differs in structure from the polycyclic musk compounds by not having a benzene ring but the properties would probably resemble those of the polycyclic musks. All compounds show low water solubility and high $\log \mathrm{K}_{\text {ow }}$ value indicating that the musk compounds are likely to accumulate in tissues with high lipid content. 
Table 3.2 Partition coefficients (octanol/water, log $\mathrm{K}_{\mathrm{ow}}$ ) water solubility and molecular weight of musk compounds.

\begin{tabular}{|c|c|c|c|c|}
\hline Compounds & $\log K_{o w}$ & $\begin{array}{l}\text { Water solubility } \\
(\mathrm{mg} / \mathrm{l})\end{array}$ & $\begin{array}{l}\text { Molecular weight } \\
\qquad(\mathrm{g} / \mathrm{mol})\end{array}$ & $\begin{array}{c}\text { Vapor pressure } \\
\text { (Pa) }\end{array}$ \\
\hline ADBI & 5.9 & 0.22 & 244 & \\
\hline AHDI & 5.85 & 0.25 & 244 & \\
\hline ATII & 6.3 & 0.09 & 258 & \\
\hline MX & 4.3 & 0.46 & 294.3 & $0.03 \times 10^{-3}$ \\
\hline $\mathrm{HHCB}$ & 5.9 & 1.65 & 258.4 & 0.068 \\
\hline AHTN & 5.7 & 1.22 & 258.4 & 0.073 \\
\hline MK & 4.3 & 0.46 & 294.3 & $0.04 \times 10^{-3}$ \\
\hline
\end{tabular}

Nylander 2001

\subsection{Musk compounds in the Nordic countries}

Table 3.3 displays recent findings of synthetic musk compounds in the Nordic countries while Table 3.4 displays findings in other countries.

Emphasis has been put on samples similar to those in the current project from other European countries and a few data on sewage treatment plants in the USA.

EU 2002 and EU 2003 refer data on toxicity of musk ketone (MK) and musk xylene (MX) respectively. NOEC (No Observed Effects Concentration) is $0.088 \mathrm{mg} / \mathrm{l}(\mathrm{MK})$ and $0.56 \mathrm{mg} / \mathrm{l}(\mathrm{MX})$ for the algae Selenastrum capricornutum, $0.17 \mathrm{mg} /(\mathrm{MK})$ and $0.056 \mathrm{mg} / \mathrm{l}(\mathrm{MX})$ for the crustacea Daphnia magna reproduction test, $0.063 \mathrm{mg} / \mathrm{l}(\mathrm{MK})$ for rainbow trout (21-d flow through) and $<0.1 \mathrm{mg} / \mathrm{l}$ (MX) for zebrafish (14 d semistatic). NOEC is the most sensitive toxicity end point. 


\begin{tabular}{|c|c|c|c|c|c|c|c|c|c|c|c|c|c|c|c|c|c|c|}
\hline & 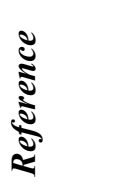 & & 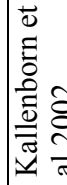 & 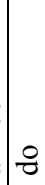 & 요 & & \& & 용 & 음 & 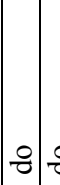 & ৪) & 8 & 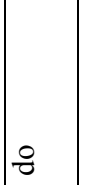 & & 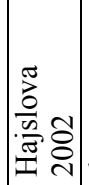 & 8 & 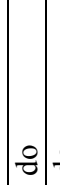 & 욤 \\
\hline & $\sum_{2}^{\infty}: 0$. & & & & & & & & & & & & & & $\stackrel{\widetilde{g}}{=}$ & $\stackrel{\overbrace{}}{=}$ & & $\stackrel{\widetilde{g}}{=}$ \\
\hline & 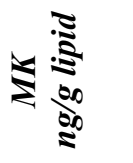 & & 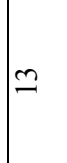 & - & ذ্ & & $?$ & $\stackrel{m}{I}$ & 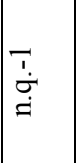 & $\stackrel{\widetilde{s}}{=}$ & 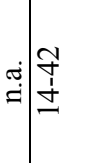 & 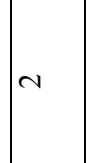 & $\frac{1}{0}$ & & $\ddot{\dot{I}}$ & 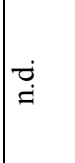 & & 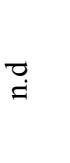 \\
\hline & 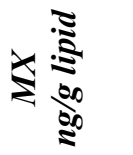 & & $\infty$ & - & $\stackrel{\infty}{\Xi}$ & & $\overrightarrow{0}$ & m & $\stackrel{+}{\dot{a}}$ & $\stackrel{\Xi}{=}$ & 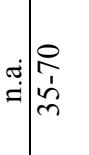 & $\nabla$ & مि & & ت্் & $\stackrel{\vec{I}}{\leftrightarrows}$ & & $\begin{array}{l}\infty \\
\vec{v} \\
\dot{v} \\
\stackrel{u}{u}\end{array}$ \\
\hline & 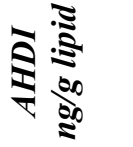 & & $\stackrel{\Xi}{=}$ & 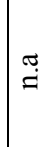 & $\stackrel{\mathscr{I}}{=}$ & & 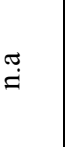 & $\stackrel{\pi}{=}$ & $\stackrel{\pi}{=}$ & $\begin{array}{l}\stackrel{\pi}{=} \\
=\end{array}$ & 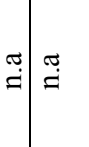 & $\stackrel{\pi}{=}$ & $\stackrel{\check{I}}{=}$ & & $\ddot{d}$ & ت્j & & త্் \\
\hline & 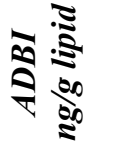 & & $\stackrel{\pi}{=}$ & 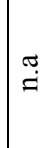 & $\stackrel{\pi}{=}$ & & تֶ & $\stackrel{\pi}{=}$ & $\stackrel{\pi}{=}$ & $\stackrel{\pi}{=}$ & 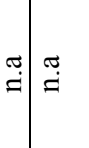 & $\stackrel{\pi}{=}$ & $\underset{\pi}{=}$ & & $\ddot{\dot{I}}$ & $\stackrel{\check{I}}{\dot{I}}$ & & 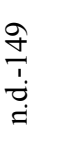 \\
\hline & 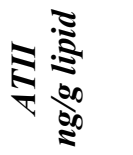 & & 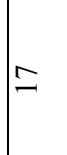 & $N$ & क人 & & ì & $\mid \begin{array}{l}\vec{I} \\
\dot{\square} \\
\dot{g}\end{array}$ & $\hat{i}$ & $=-$ & $-\begin{array}{l}a \\
\hat{y} \\
n\end{array}$ & ঙ্ & 妾 & & ¿ே. & $\ddot{\check{I}}$ & & 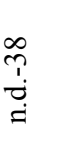 \\
\hline & 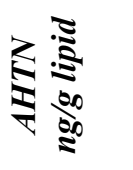 & & $\infty$ & $m$ & 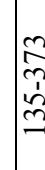 & & 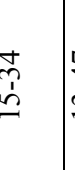 & $\begin{array}{l}\mathfrak{y} \\
\dot{1} \\
\end{array}$ & $\frac{0}{b}$ & $\approx-$ & $-\mid \begin{array}{l}\stackrel{\infty}{\infty} \\
\frac{1}{\infty}\end{array}$ & \pm & $\frac{m}{m}$ & & 离 & $\begin{array}{l}w \\
\alpha \\
\infty \\
\infty \\
\vec{v}\end{array}$ & $\begin{array}{cc}\vec{F} \\
\dot{b} \\
\infty \\
\vec{v} \\
0 \\
0\end{array}$ & $\begin{array}{l}\hat{b} \\
\text { b. } \\
\infty \\
\vec{v}\end{array}$ \\
\hline & 离 & & $\cong$ & $\vec{N}$ & 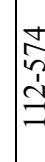 & d & 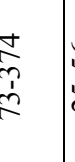 & $\begin{array}{l}0 \\
0 \\
n \\
i n \\
i n\end{array}$ & $\begin{array}{l}\text { qे } \\
\text { ஸे }\end{array}$ & הิ & 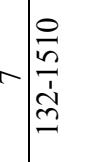 & $\lesssim$ & $\vec{a}$ & & 命 & 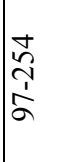 & 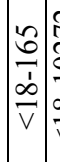 & $\begin{array}{l}\underset{\widetilde{d}}{\sigma} \\
\frac{1}{\infty} \\
\overrightarrow{0} \\
v\end{array}$ \\
\hline & 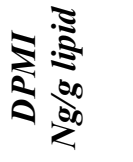 & & $\stackrel{\pi}{\stackrel{I}{\leftrightarrows}}$ & 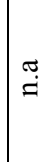 & $\stackrel{\mathscr{I}}{=}$ & & 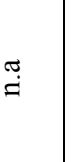 & $\stackrel{\pi}{=}$ & $\stackrel{\pi}{ت}$ & $\begin{array}{l}\tilde{g} \\
\dot{\Xi}\end{array}$ & 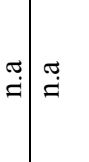 & $\stackrel{\pi}{=}$ & 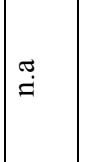 & & $\stackrel{\pi}{\stackrel{I}{=}}$ & $\stackrel{\pi}{\leftrightarrows}$ & & $\stackrel{\pi}{=}$ \\
\hline & 胥 & & 象 & 吕 & ?ִ & $\begin{array}{l}3 \\
8 \\
5 \\
5 \\
5\end{array}$ & $\begin{array}{l}\frac{1}{0} \\
\frac{1}{0} \\
\dot{0}\end{array}$ & ج̂ & 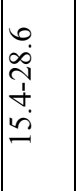 & $\stackrel{m}{i}$ & 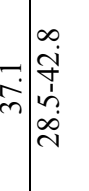 & $\stackrel{n}{=}$ & 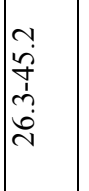 & & $\begin{array}{l}\hat{2} \\
0 \\
0 \\
0 \\
\vdots \\
0 \\
0\end{array}$ & $\begin{array}{l}n \\
2 \\
0 \\
1 \\
\vdots \\
0 \\
0\end{array}$ & 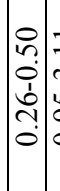 & $\begin{array}{l}= \\
\dot{r} \\
\dot{n} \\
\vdots \\
0\end{array}$ \\
\hline$E$ & 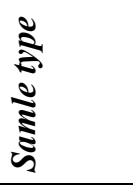 & & 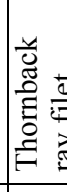 & & 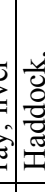 & 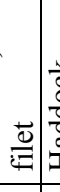 & 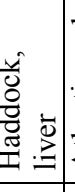 & 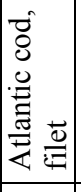 & 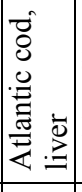 & 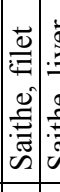 & 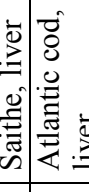 & 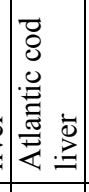 & 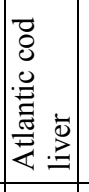 & & 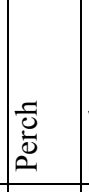 & $\begin{array}{l}-\tilde{0} \\
\overline{0} \\
0 \\
0 \\
\end{array}$ & 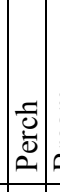 & 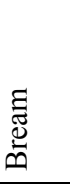 \\
\hline & : & 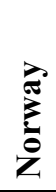 & 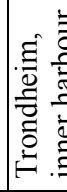 & 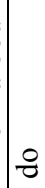 & $\frac{8}{8}$ & & ৪ & 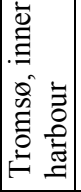 & 8 & 8 & 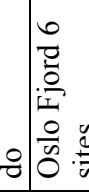 & 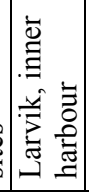 & 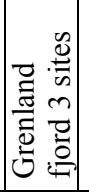 & 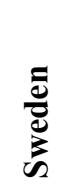 & 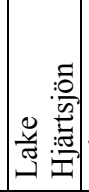 & 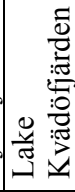 & 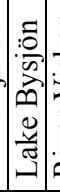 & 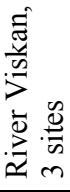 \\
\hline
\end{tabular}




\begin{tabular}{|c|c|c|c|c|c|c|c|c|}
\hline 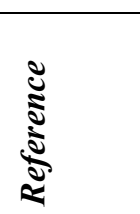 & $\because$ & 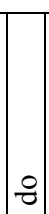 & \& & 8 & & 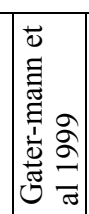 & 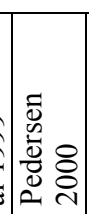 & 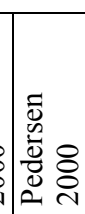 \\
\hline$Z^{2}=0$ & 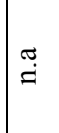 & $\mid \begin{array}{c}\Im \\
\dot{=}\end{array}$ & $\stackrel{\pi}{=}$ & $\stackrel{\pi}{=}$ & & 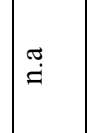 & 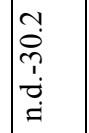 & 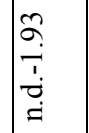 \\
\hline 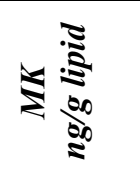 & $\stackrel{\vec{\jmath}}{\ddot{\prime}}$ & $\stackrel{i}{\dot{a}}$ & تُ & $\mid \begin{array}{r}\dot{\mu} \\
\dot{\vec{g}}\end{array}$ & & \begin{tabular}{l}
1 \\
\multirow{1}{0}{} \\
0 \\
0 \\
0 \\
0 \\
0
\end{tabular} & 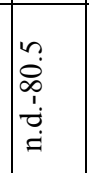 & 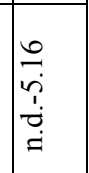 \\
\hline 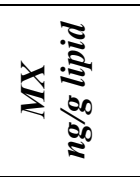 & $\stackrel{\vec{j}}{\ddot{\prime}}$ & ت己 & İ & $\stackrel{\overrightarrow{\dot{A}}}{\dot{s}}$ & & $\begin{array}{c}\overline{\hat{\gamma}} \\
0 \\
0 \\
0 \\
0 \\
0\end{array}$ & 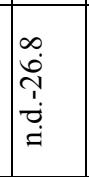 & 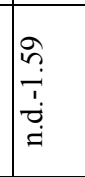 \\
\hline 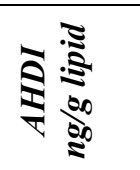 & تே & ت்ְ & تُ & $\mid \begin{array}{r}\dot{\Xi} \\
\dot{=}\end{array}$ & & $\stackrel{\overparen{J}}{=}$ & 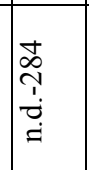 & 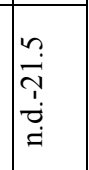 \\
\hline 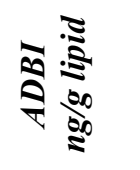 & $\stackrel{\vec{J}}{\ddot{\prime}}$ & $\stackrel{\vec{i}}{=}$ & تُ & $\dot{\overrightarrow{\dot{C}}}$ & & 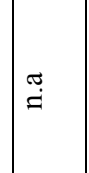 & 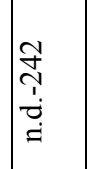 & 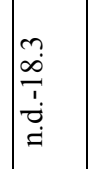 \\
\hline 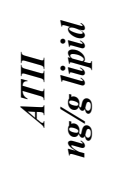 & $\stackrel{\vec{j}}{\ddot{\prime}}$ & $\stackrel{\vec{D}}{=}$ & تُ & $\stackrel{\dot{\oplus}}{\dot{=}}$ & & 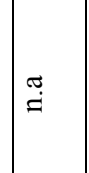 & 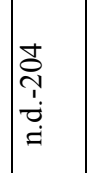 & 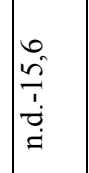 \\
\hline 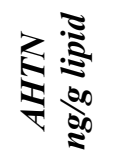 & 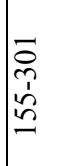 & 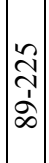 & $\begin{array}{l}\stackrel{+}{1} \\
\frac{1}{d} \\
\text { I }\end{array}$ & 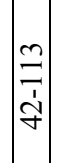 & & $\begin{array}{l}\hat{n} \\
\hat{0} \\
\vdots \\
\grave{1} \\
0 \\
0\end{array}$ & $\begin{array}{l}\tilde{N} \\
\hat{2} \\
\hat{2} \\
\text { in } \\
\text { in }\end{array}$ & 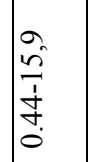 \\
\hline 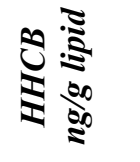 & 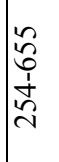 & 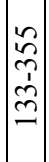 & 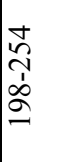 & $\mid$\begin{tabular}{c}
$\infty$ \\
\multirow{2}{1}{} \\
$\grave{1}$ \\
$\infty$ \\
$\infty$
\end{tabular} & & $\mid \begin{array}{l}0 \\
0 \\
0 \\
1 \\
\\
0 \\
0\end{array}$ & 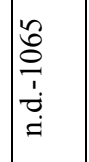 & $\mid \begin{array}{l}0 \\
i \\
i \\
i \\
\dot{j} \\
=\end{array}$ \\
\hline 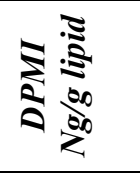 & 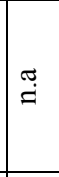 & $\begin{array}{l}\stackrel{\pi}{二} \\
\end{array}$ & $\underset{\pi}{=}$ & $\stackrel{\Xi}{=}$ & & 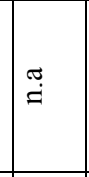 & $\begin{array}{l}\hat{A} \\
\hat{N} \\
\hat{i} \\
\dot{y} \\
\dot{y}\end{array}$ & $\stackrel{\pi}{=}$ \\
\hline 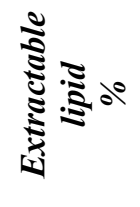 & 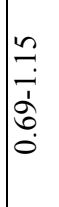 & 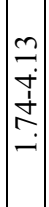 & 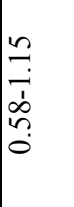 & $\mid \begin{array}{l}\hat{\alpha} \\
\hat{a} \\
\grave{n} \\
\\
i\end{array}$ & & 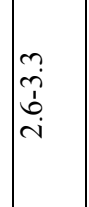 & 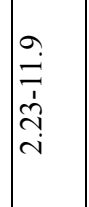 & 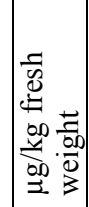 \\
\hline 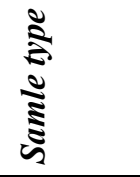 & 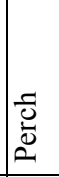 & 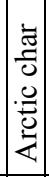 & $\begin{array}{l} \\
\\
0 \\
0 \\
0 \\
0 \\
\end{array}$ & 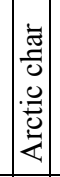 & & 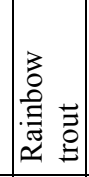 & 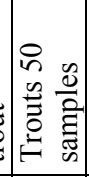 & 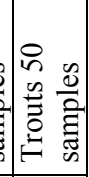 \\
\hline : & 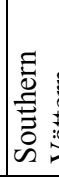 & & 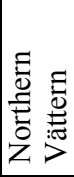 & 8 & & 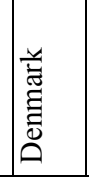 & 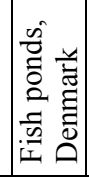 & 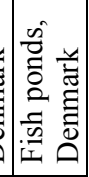 \\
\hline
\end{tabular}

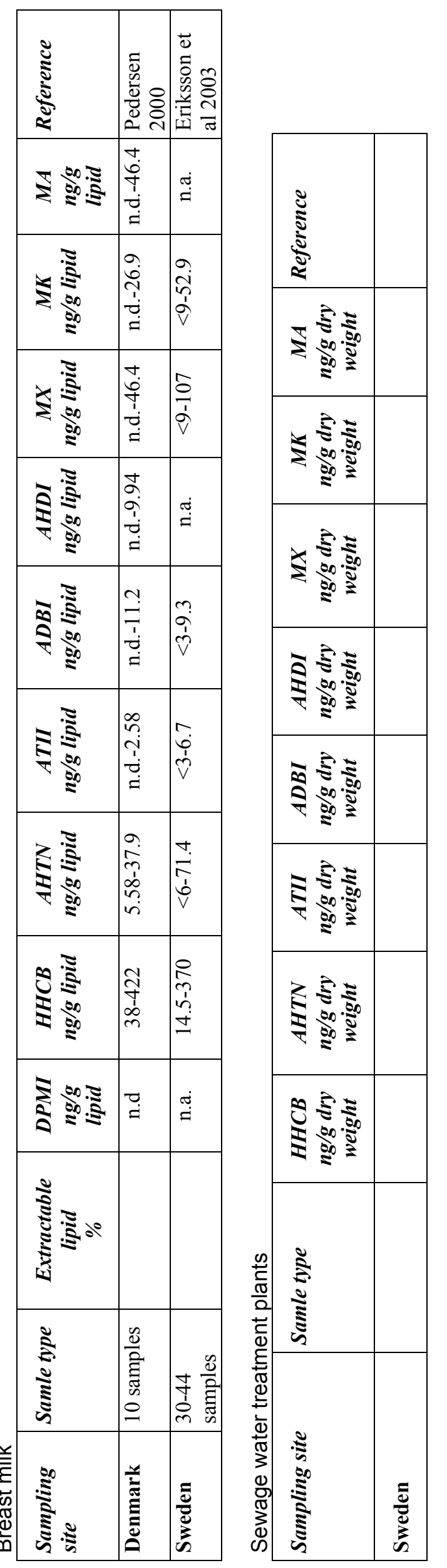




\begin{tabular}{|c|c|c|c|c|c|c|c|c|c|c|c|c|c|c|c|c|c|c|}
\hline & & & 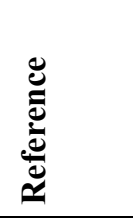 & & 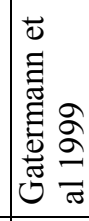 & 8 & 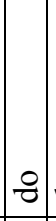 & 8 & 8 & 8. & 8 & 8 & 8 & $\because$ & 8 & $8 \%$ & 8 & \\
\hline \multirow{2}{*}{ 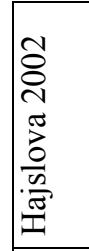 } & \multirow[b]{2}{*}{$\%$} & $\begin{array}{l}\frac{\pi}{0} \\
\frac{\pi}{0} \\
0 \\
\frac{0}{5}\end{array}$ & 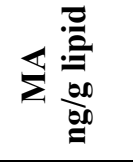 & & 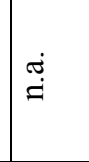 & 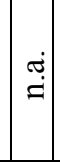 & $\stackrel{\stackrel{\leftrightarrow}{\dot{g}}}{\mathrm{~g}}$ & 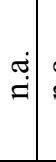 & $\stackrel{\oplus}{=}$ & تே & 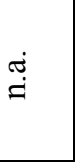 & 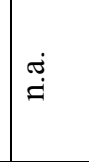 & $\stackrel{\pi}{=}$ & 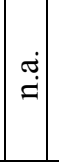 & $\stackrel{\stackrel{\leftrightarrow}{\sharp}}{=}$ & 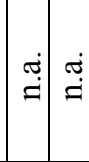 & $\stackrel{\leftrightarrow}{=}$ & \\
\hline & & $\frac{\pi}{\sigma}$ & . & & & & & क) & $\approx$ & $\because 8$ & & $\mathscr{F}$ & & & & $\nexists{ }^{\top}$ & & \\
\hline$\stackrel{\pi}{=}$ & $\stackrel{\leftrightarrow}{\stackrel{\leftrightarrow}{=}}$ & $\begin{array}{l}\frac{E}{\Xi} \\
0 \\
0\end{array}$ & 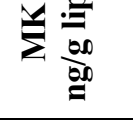 & & 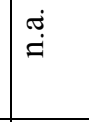 & $\stackrel{\oplus}{\dot{\oplus}}$ & 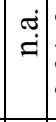 & 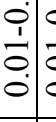 & $\begin{array}{c}0 \\
0 \\
\vdots \\
0 \\
0 \\
0\end{array}$ & & 官 & $\begin{array}{l}\dot{0} \\
\stackrel{1}{\Delta} \\
\stackrel{0}{0}\end{array}$ & $\stackrel{\overbrace ্}{=}$ & & $\begin{array}{l}\ddot{0} \\
1 \\
n \\
\tilde{n} \\
0\end{array}$ & 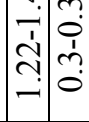 & $\stackrel{\infty}{\stackrel{\infty}{\sim}}$ & 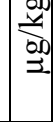 \\
\hline ¿्் & $\stackrel{\vec{I}}{\dot{I}}$ & 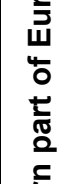 & 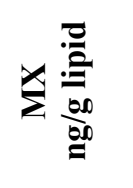 & & $\stackrel{\oplus}{=}$ & 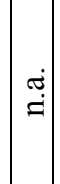 & $\stackrel{\stackrel{\leftrightarrow}{\dot{g}}}{=}$ & 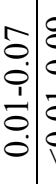 & 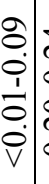 & 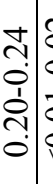 & $\begin{array}{l}\text { ô } \\
\dot{0} \\
1 \\
\vdots \\
0 \\
\dot{v}\end{array}$ & $\begin{array}{l}\overrightarrow{0} \\
\dot{0} \\
\dot{1} \\
0 \\
\dot{0} \\
\dot{v}\end{array}$ & 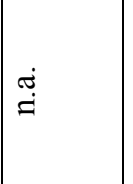 & $\stackrel{\leftrightarrow}{\stackrel{\leftrightarrow}{\dot{d}}}$ & $\begin{array}{l}\hat{N} \\
0 \\
\dot{1} \\
\\
0\end{array}$ & 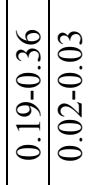 & $\frac{1}{0}$ & 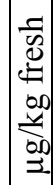 \\
\hline $\begin{array}{l}\sim \\
\stackrel{n}{n} \\
\end{array}$ & 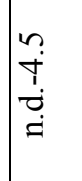 & 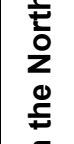 & $\overline{\mathrm{E}}$ & & 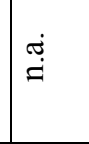 & $\stackrel{\oplus}{=}$ & : & $\stackrel{\oplus}{\dot{y}}$ & تே & $\stackrel{\oplus}{\dot{\varphi}}$ & త্் & 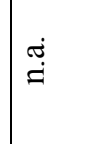 & $\stackrel{\overbrace \underset{j}{g}}{=}$ & $\mid \begin{array}{r}\stackrel{\leftrightarrow}{二} \\
=\end{array}$ & 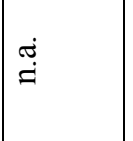 & 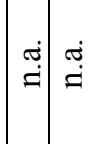 & $\stackrel{\leftrightarrow}{\operatorname{I}}$ & \\
\hline ت. & $\stackrel{\dot{I}}{\dot{I}}$ & 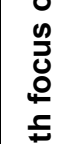 & $\overline{\mathrm{Q}}$ & & 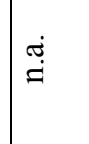 & 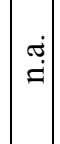 & $\stackrel{\stackrel{\leftrightarrow}{\dot{g}}}{=}$ & 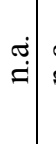 & 迅| & $\stackrel{\oplus}{\dot{g}}$ & $\stackrel{\leftrightarrow}{\dot{g}}$ & 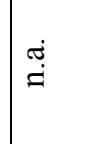 & $\stackrel{\overbrace \underset{j}{g}}{=}$ & 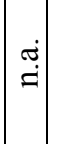 & $\stackrel{\stackrel{\leftrightarrow}{\leftrightarrows}}{\stackrel{\Xi}{=}}$ & 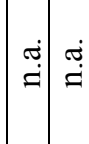 & 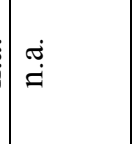 & 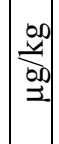 \\
\hline$\frac{a}{\frac{1}{6}}$ & $\begin{array}{c}\hat{T} \\
\infty \\
\infty\end{array}$ & 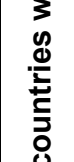 & 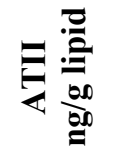 & & $\stackrel{\pi}{=}$ & ت্் & 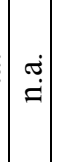 & 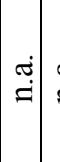 & 迎| & 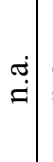 & ঙ্ं & 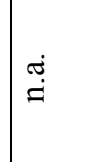 & $\stackrel{\pi}{=}$ & $\mid \begin{array}{c}\stackrel{\leftrightarrow}{\sharp} \\
\stackrel{=}{\mid}\end{array}$ & 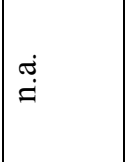 & 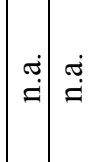 & 状 & \\
\hline$\exists$ & $\begin{array}{c}\tilde{N} \\
\tilde{n}\end{array}$ & $\begin{array}{l}0.0 \\
0 \\
0 \\
2 \\
0 \\
\underline{0} \\
0\end{array}$ & 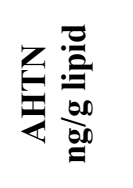 & & $\begin{array}{l}\vec{i} \\
\stackrel{i}{\sim}\end{array}$ & $\begin{array}{l}0 \\
0 \\
n \\
n \\
n \\
n\end{array}$ & $\begin{array}{l}n \\
0 \\
0 \\
1 \\
0 \\
0\end{array}$ & 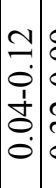 & 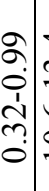 & 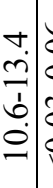 & 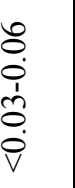 & $\begin{array}{l}0 \\
\stackrel{0}{0} \\
0 \\
\dot{0} \\
\dot{0} \\
\dot{0}\end{array}$ & 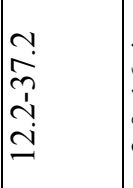 & 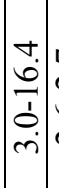 & $\begin{array}{l}\hat{i} \\
\text { bे } \\
\text { in }\end{array}$ & 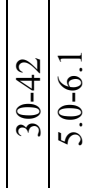 & $\mathscr{v}$ & 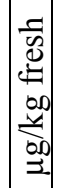 \\
\hline $\begin{array}{l}\frac{\delta}{0} \\
1 \\
\infty \\
\infty\end{array}$ & 旁 & 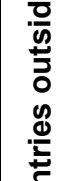 & 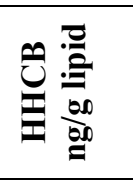 & & $\begin{array}{l}\infty \\
\dot{n} \\
\infty \\
i \\
i\end{array}$ & $\begin{array}{l}m \\
n \\
n \\
n \\
i \\
n\end{array}$ & $\begin{array}{l}0 \\
0 \\
\dot{0} \\
\dot{1} \\
\dot{0}\end{array}$ & 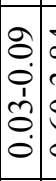 & 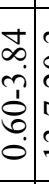 & 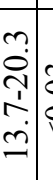 & 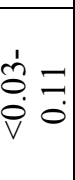 & $\mid \begin{array}{ll}1 & \\
0 & n \\
\dot{0} & 0 \\
V & 0\end{array}$ & $\mid$\begin{tabular}{l}
$\infty$ \\
\hdashline \\
\\
0 \\
0
\end{tabular} & $\left.\mid \begin{array}{c}0 \\
0 \\
0 \\
0 \\
-1\end{array}\right]$ & $\begin{array}{l}\infty \\
\stackrel{+}{+} \\
\dot{\sigma} \\
\dot{\sigma}\end{array}$ & 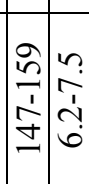 & ำ & 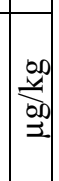 \\
\hline \begin{tabular}{l}
$\hat{m}$ \\
\multirow{2}{*}{} \\
\\
$\hat{m}$
\end{tabular} & $\begin{array}{l}\tilde{N} \\
\infty \\
1 \\
\tilde{N} \\
\tilde{\sigma}\end{array}$ & 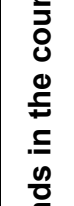 & 总 & & $\begin{array}{l}0 \\
n \\
n \\
n \\
0 \\
0\end{array}$ & 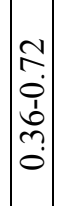 & 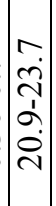 & & 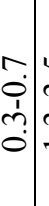 & 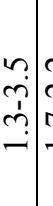 & 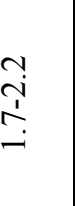 & $\frac{0}{\dot{i}}$ & 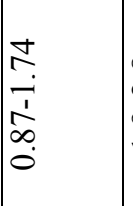 & 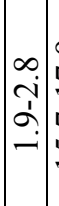 & $\begin{array}{l}\frac{9}{5} \\
\frac{1}{1} \\
i n \\
2\end{array}$ & 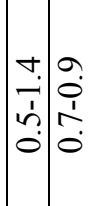 & $\stackrel{\nabla}{-}$ & \\
\hline 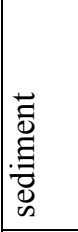 & 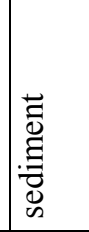 & 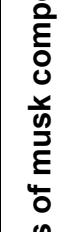 & 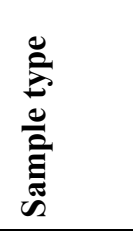 & & 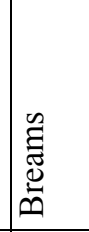 & $\mid \begin{array}{l}-\frac{\pi}{0} \\
0 \\
0 \\
2 \\
\end{array}$ & $\overrightarrow{0}$ & & 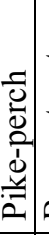 & & 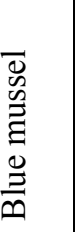 & 言 & 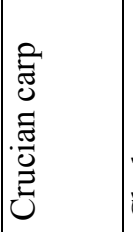 & 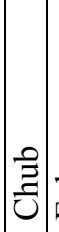 & $\overline{0}$ & 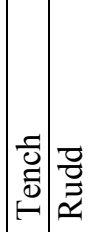 & $\mid \begin{array}{l}n \\
0 \\
0 \\
0 \\
0 \\
\vdots \\
\vdots\end{array}$ & \\
\hline 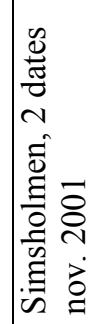 & 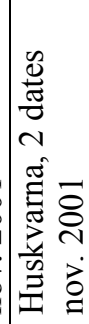 & 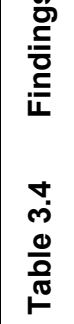 & 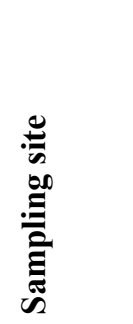 & 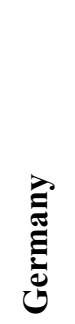 & $\begin{array}{l}\vec{z} \\
\overline{\vec{z}} \\
\overline{\bar{\alpha}} \\
\overrightarrow{\underline{\alpha}}\end{array}$ & $\stackrel{\circ}{\circ}$ & 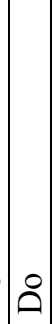 & 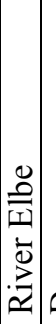 & & & & ค̊ & 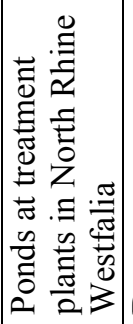 & ๑. & 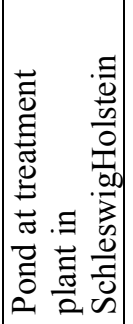 & ค̊ & 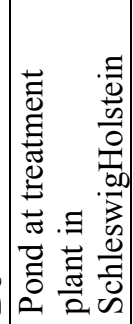 & \\
\hline
\end{tabular}




\begin{tabular}{|c|c|c|c|c|c|}
\hline 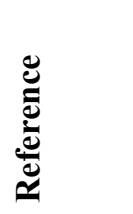 & & 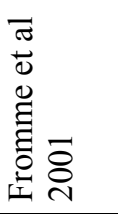 & 용 & $\div$ & 웅 \\
\hline 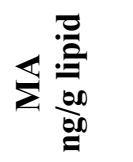 & & $\stackrel{\stackrel{\Perp}{\sharp}}{=}$ & 苛 & 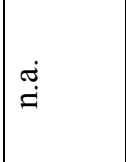 & 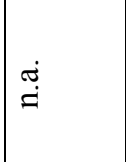 \\
\hline 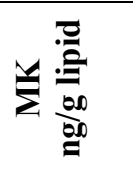 & 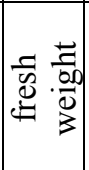 & 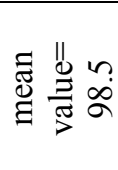 & 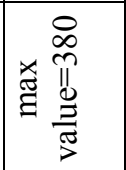 & 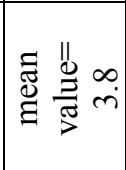 & 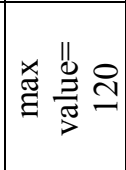 \\
\hline 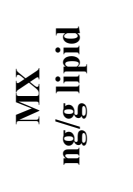 & $\mid \begin{array}{l}\frac{7}{6} \\
.000 \\
00 \\
3 \\
3\end{array}$ & 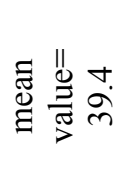 & 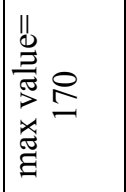 & 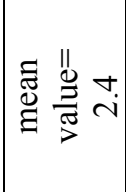 & 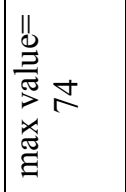 \\
\hline$\widehat{\overline{\mathbf{T}}}$ & & $\stackrel{\stackrel{\leftrightarrow}{\sharp}}{=}$ & 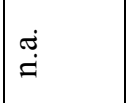 & $\stackrel{\stackrel{\leftrightarrow}{\sharp}}{=}$ & $\stackrel{\stackrel{\leftrightarrow}{\sharp}}{=}$ \\
\hline 商 & 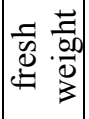 & 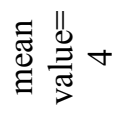 & 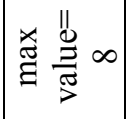 & تُ & ટ் \\
\hline 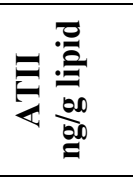 & & $\stackrel{\stackrel{\leftrightarrow}{\sharp}}{=}$ & 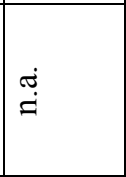 & 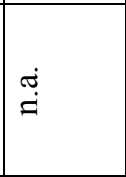 & 异 \\
\hline 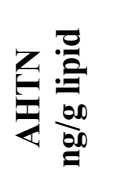 & $\mid \begin{array}{l}\frac{7}{6} \\
\frac{.00}{0} \\
\frac{1}{3}\end{array}$ & 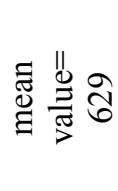 & 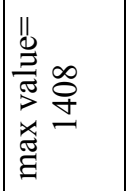 & 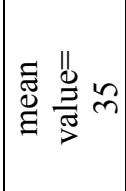 & 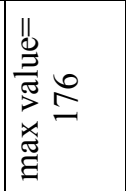 \\
\hline 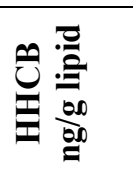 & 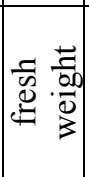 & 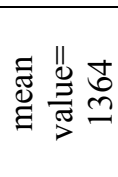 & 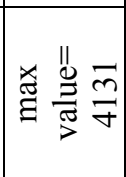 & 离 $\frac{\|}{\Xi}$ & 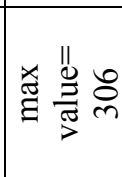 \\
\hline 总 & & & & & \\
\hline 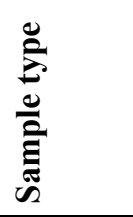 & & 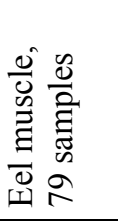 & 응 & 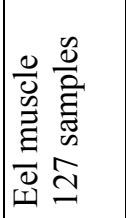 & 요 \\
\hline 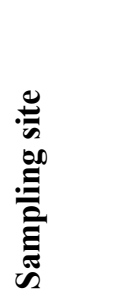 & & 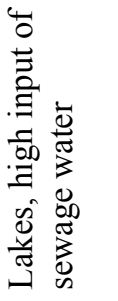 & $\stackrel{\circ}{\circ}$ & 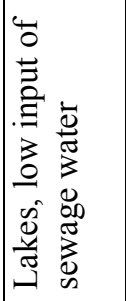 & $\stackrel{\circ}{\circ}$ \\
\hline
\end{tabular}

\begin{tabular}{|c|c|c|c|}
\hline 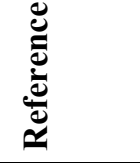 & 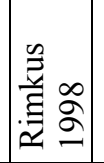 & & \\
\hline 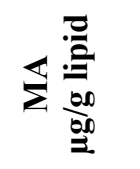 & $\stackrel{\overbrace{}}{\stackrel{\oplus}{\leftrightarrows}}$ & 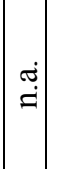 & 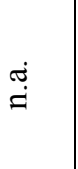 \\
\hline 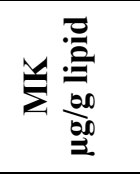 & 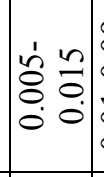 & \begin{tabular}{|l|} 
\\
$\dot{0}$ \\
0 \\
$\dot{1}$ \\
$\dot{0}$ \\
0 \\
0
\end{tabular} & $\begin{array}{cc}1 & \overrightarrow{8} \\
8 & \overline{0} \\
0 & 0 \\
0 & 0\end{array}$ \\
\hline 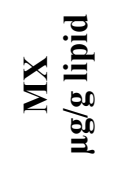 & $\begin{array}{l}0 \\
0 \\
\dot{1} \\
0 \\
0 \\
0\end{array}$ & $\mid$\begin{tabular}{c}
0 \\
\hdashline \\
0 \\
1 \\
$\vdots$ \\
0 \\
0 \\
0
\end{tabular} & 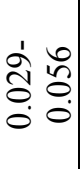 \\
\hline 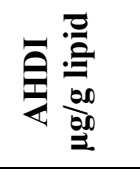 & $\stackrel{ه ্}{=}$ & $\mid \stackrel{\stackrel{\oplus}{\sharp}}{=}$ & 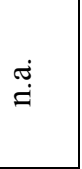 \\
\hline 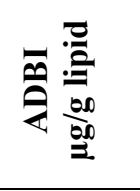 & $\begin{array}{l}\infty \\
0 \\
0 \\
\dot{1} \\
\dot{1} \\
0 \\
0 \\
0\end{array}$ & $\stackrel{\leftrightarrow}{=}$ & 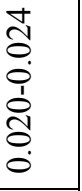 \\
\hline 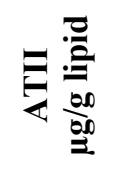 & 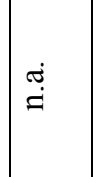 & 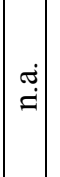 & 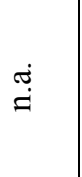 \\
\hline 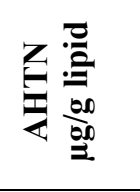 & $\begin{array}{l}\infty \\
0 \\
0 \\
0 \\
1 \\
\\
0 \\
0\end{array}$ & $\stackrel{\leftrightarrow}{=}$ & 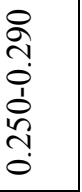 \\
\hline 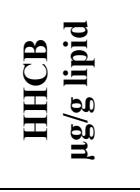 & $\begin{array}{l}0 \\
0 \\
0 \\
0 \\
0 \\
0 \\
0 \\
0 \\
0\end{array}$ & $\stackrel{\leftrightarrow}{=}$ & $\begin{array}{l}0 \\
0 \\
? \\
0 \\
0 \\
0 \\
m \\
0\end{array}$ \\
\hline 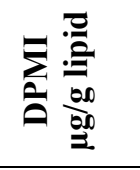 & & $\mid \begin{array}{c}\stackrel{g}{\dot{g}} \\
\end{array}$ & 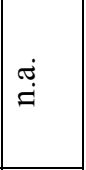 \\
\hline 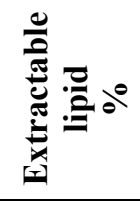 & & & \\
\hline z & in & $\approx$ & $\sim$ \\
\hline 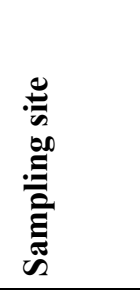 & 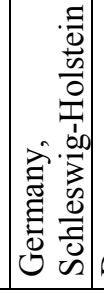 & 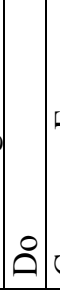 & 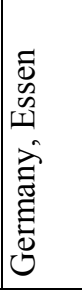 \\
\hline
\end{tabular}




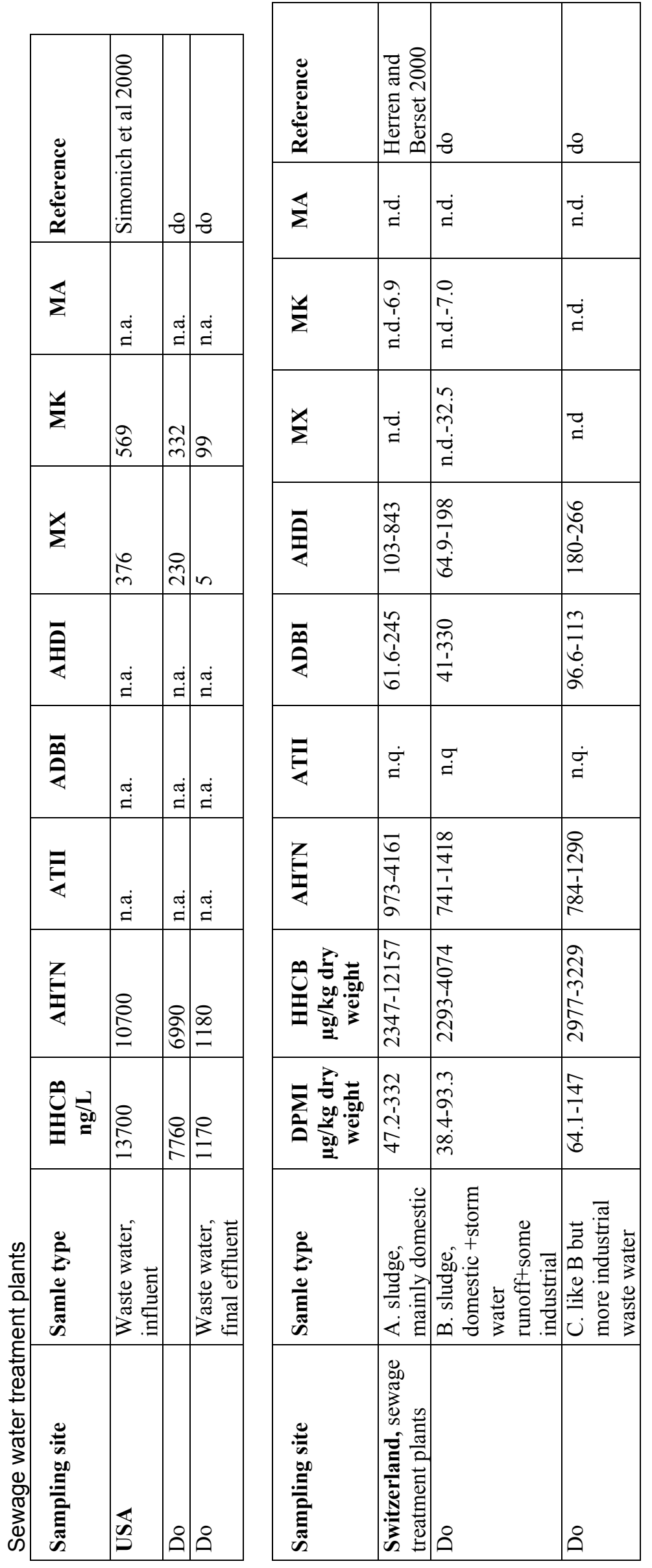




\section{Samples from the Nordic Countries}

\subsection{Choice of matrix}

Musk compounds are introduced into the environment from sewage water. They are partly retained and degraded in sewage treatment plants. During treatment in the plants musks may be released to the air. Effluents from treatment plants are a major source of synthetic musk compounds to the aquatic environment and can be accumulated in aquatic organisms. Musk compounds are further released directly to air from cosmetics and household products where musk compounds are used as fragrances. Rainwater may wash musk compounds from the atmosphere. Humans are exposed to musk compounds directly from cosmetics and washed fabrics and the compounds may end up in fat tissues and in breast milk. Musk compounds may enter the terrestrial food chain from the above mentioned sources and via household waste. Foxes in urban areas feed partly on garbage from households. They are also predators and may accumulate musk compounds via the food chain.

Based upon the above considerations the NTEM working group decided to focus on the following matrices for screening of musk compounds: Sludge from waste water treatment plants, rain water, blue mussels and fox liver. Sampling sites should preferably represent urban areas as well as more pristine areas. Breast milk was also considered an important matrix. However, the available budget and the cost of analysis made it necessary to prioritise the choice of matrix. In the final sampling programme breast milk was therefore not included in the monitoring programme.

\subsection{Sample collection}

A sampling manual was distributed to all members of the steering group prior to the sample collection took place. The sampling manual is attached as annex 1.Each of the Nordic Countries was responsible for collecting samples and sending the samples to NERI for analysis.

It has not been possible to collect all samples from all countries. Summer 2002 was very dry in Scandinavia making it difficult to collect rainwater. Further, it turned out to be difficult to get the proposed number of fox liver samples.

Figure 4.1 provides an overview of the sampling sites and Annex 2 contains the information provided from each country. 


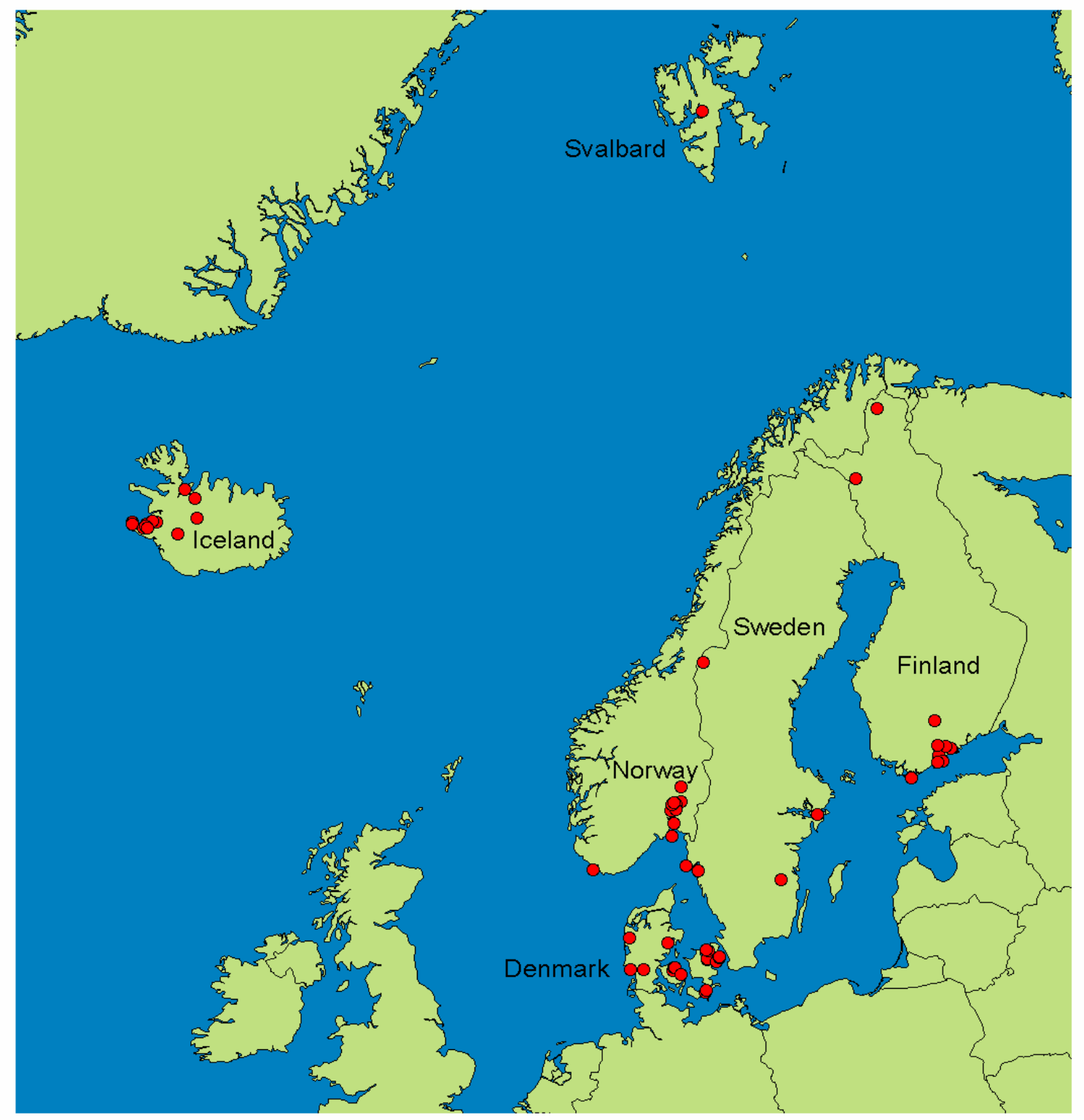

Figure 4.1 All sampling sites from the Nordic countries. Each sampling station is indicated by a red circle. 


\subsection{Methods of analysis}

\subsubsection{Principles}

The analytical method consists of an extraction and cleanup step and a chromatographic analysis. Extraction and cleanup differs according to sample type. Water samples were liquid-liquid extracted, sludge samples were extracted by shaking the samples with solvent and biota samples were extracted by soxhlet extraction. All detection was carried out by GC-MS. ${ }^{14} \mathrm{C}$ marked Tonalide and Musk xylene were used as recovery standard in all samples. Alfa-amyl cinnamic aldehyde was used as an internal standard in some diluted sludge samples and biota samples.

\subsubsection{Extraction methods}

\section{Rain water}

Water samples: $250 \mu 1$ of recovery standard (200 $\mathrm{ng} / \mathrm{ml})$ were added to $1000 \mathrm{ml}$ of rainwater. The water was shaken manually with dichloromethane $(100+100+50 \mathrm{ml})$. The extracts were transferred to a round bottom flask through anhydrous sodium sulphate. The combined extracts were reduced by rotary evaporation $\left(30^{\circ} \mathrm{C}\right)$ to $4-5 \mathrm{ml}$ and transferred to $10 \mathrm{ml}$ volumetric centrifuge tubes. Volume was reduced to $500 \mu \mathrm{l}$ under nitrogen at room temperature using toluene as a keeper. Volume was adjusted to 1 $\mathrm{ml}$ with toluene.

\section{Sewage sludge}

$10 \mathrm{~g}$ of sewage sludge was extracted with $150 \mathrm{ml}$ of dichloromethane:methanol (80:20) by initial sonification for 10 minutes followed by 2 hours of shaking on a shaking table. Before extraction, $250 \mu \mathrm{l}$ of recovery standard $(200 \mathrm{ng} / \mathrm{ml})$ was added to the sludge. The extract was decanted through anhydrous sodium sulphate into a round bottom flask. The volume was reduced by rotary evaporation to $500 \mu \mathrm{l}$ using toluene as a keeper. The extract was cleaned up by solid phase extraction on silica cartridges from International Sorbent Technology, $2 \mathrm{~g}$ of silica gel in glass tubes. The columns were conditioned with dichloromethane and pentane. After application of the sample, the column was rinsed with $5 \mathrm{ml}$ of pentane and eluted with $10 \mathrm{ml}$ of dihloromethane:methanol (80:20). The volume was reduced under nitrogen at room temperature to $500 \mu \mathrm{l}$ using toluene as a keeper. Volume was adjusted to $1 \mathrm{ml}$.

Some of the extracts showed high concentrations of some of the musk compounds. They were diluted and reanalysed.

\section{Biota}

All blue mussels from each sampling site were homogenised using ultraturrax homogenizer. Likewise each whole liver sample was homogenised. The homogenised samples were kept frozen $\left(-18^{\circ} \mathrm{C}\right)$ until extraction. $10 \mathrm{~g}$ of blue mussel or $2 \mathrm{~g}$ of fox liver was soxhlet extracted with $300 \mathrm{ml}$ of dichloromethane: methanol (80:20) for 16 hours. Before extraction, $200 \mu \mathrm{l}$ of recovery standard $200 \mathrm{ng} / \mathrm{ml}$ were added to the sample. The extract was filtered through anhydrous sodium sulphate into a round bottom flask and the volume was reduced by rotary evaporation to about $50 \mathrm{ml}$. The extract was transferred to a measuring flask and the volume was adjusted to $100 \mathrm{ml} .10$ $\mathrm{ml}$ of the extract was used for determination of the fat content. The remaining $90 \mathrm{ml}$ were further reduced to $2 \mathrm{ml}$. An aliquot of the extract, 1000 or $250 \mu 1$ of respectively 
mussel and liver extract were cleaned up to remove the fat from the extract using a Phenogel GPC (Gel Permeation Chromatography) column 300x21.2 mm packed with a styrene-divinylbenzene polymer, $10 \mu$ particle size, $100 \AA$ pore size (Phenomenex). The HPLC system and fraction collector was from Gilson encompassing a 233 XL injection and collection system, a 322 pump, a 402 syringe pump and a 155 UV/VIS detector. Eluent was dichloromethane:methanol (98:2) at a flow of $4 \mathrm{ml} / \mathrm{min}$. The fraction containing the musk compounds was selected after running a $10 \mu \mathrm{g} / \mathrm{ml}$ standard solution and detecting the retention time with UV spectrometry. The $20 \mathrm{ml}$ fraction was reduced to $500 \mu \mathrm{l}$ by nitrogen using toluene as a keeper and volume adjusted to $1 \mathrm{ml}$.

\section{Detection}

Detection was carried out with GC-MS. The equipment was a Perkin Elmer Autosystem XL gas chromatograph equipped with a Perkin Elmer Turbomass quadrupole mass spectrometer. A Chrompack WCOT fused silica $50 \mathrm{~m}$ long, diameter $0.32 \mathrm{~mm}$ coated with CP-SIL $5 \mathrm{CB}$ low bleed material, film density $1.20 \mu \mathrm{m}$ was used to separate the analytes. Calibration was carried out using a cubic curve fit.

\section{GC conditions}

Splitless injection of $2 \mu 1$. Injector temperature $250^{\circ} \mathrm{C}$. Oven programme: Initial temperature $40^{\circ} \mathrm{C}, 40$ to $220^{\circ} \mathrm{C}$ at $40^{\circ} \mathrm{C} / \mathrm{min}, 220$ to $280^{\circ} \mathrm{C}$ at $2^{\circ} \mathrm{C} / \mathrm{min}$ hold for 3 minutes. Helium at a flow of $2.00 \mathrm{ml} / \mathrm{min}$. was used as carrier gas. The capillary column was coupled to the ion source $250^{\circ} \mathrm{C}$, transfer line temperature $280^{\circ} \mathrm{C}$

\section{MS conditions}

The following MS parameters were used (Table ): EI $70 \mathrm{eV}$, selected ion mode.

Table 4.1 Retention times and ions used for identification and quantification of musk compounds

\begin{tabular}{|l|c|c|c|}
\hline Compound & Ret. Time min. & Qualifier ions [m/z] & $\begin{array}{c}\text { Mass used for } \\
\text { quantification [m/z] }\end{array}$ \\
\hline Cashmeran (DPMI) & 10.93 & 163,206 & 191 \\
\hline Celestolide ADBI) & 14.47 & 173,244 & 229 \\
\hline Phantolide (AHDI) & 15.68 & 187,244 & 229 \\
\hline Musk ambrette (MA) & 17.60 & 145,268 & 253 \\
\hline Musk xylene* & 18.04 & 136,312 & 294 \\
\hline Traseolide (ATII) & 18.28 & 173,258 & 215 \\
\hline Musk xylene (MX) & 18.50 & 128,297 & 282 \\
\hline Galaxolide (HHCB) & 18.65 & 213,258 & 243 \\
\hline Tonalide* & 18.73 & 190,261 & 243 \\
\hline Tonalide (AHTN) & 18.87 & 187,258 & 279 \\
\hline Musk ketone (MK) & 22.30 & 128,294 & $\begin{array}{c}\text { Mass used for } \\
\text { quantification [m/z] }\end{array}$ \\
\hline Internal standard & Ret. time & $\begin{array}{c}\text { Qualifier ions } \\
{[\mathrm{m} / \mathrm{z}]}\end{array}$ & 115 \\
\hline $\begin{array}{l}\text { Alpha-amyl cinnamic } \\
\text { aldehyde }\end{array}$ & 12.79 & 145,202 & \\
\hline
\end{tabular}




\section{Results}

Recovery and limits of detection for each matrix are indicated in Table 5.1. Recovery is determined with samples spiked with a mixture of musk compounds in a matrix similar to the natural sample. The samples were spiked at levels below 5 times the limit of detection. Limit of detection (LOD) is calculated as three times signal to noise in blanks of each matrix taking into account sample amount and recovery. LOQ in rainwater is calculated as three times standard deviation for 6 recovery samples spiked with $50 \mathrm{ng} / 1$ of all musk compounds. In sewage sludge and biological samples LOQ is calculated as three times LOD.

Table 5.1 Detection and recovery data

\begin{tabular}{|c|c|c|c|c|c|}
\hline Matrix & Compound & Recovery \% & $\begin{array}{c}\text { Uncertainty } \\
\%\end{array}$ & LOQ & LOD \\
\hline \multirow[t]{9}{*}{$\begin{array}{l}\text { Rain water } \\
\text { ng/l }\end{array}$} & Cashmeran (DPMI) & 40 & 25 & 8 & 5 \\
\hline & Celestolide ADBI) & 47 & 24 & 14 & 5 \\
\hline & Phantolide (AHDI) & 50 & 24 & 16 & 5 \\
\hline & $\begin{array}{l}\text { Musk ambrette } \\
\text { (MA) }\end{array}$ & 53 & 19 & 11 & 10 \\
\hline & Traseolide (ATII) & 50 & 26 & 18 & 5 \\
\hline & Musk xylene (MX) & & & & 10 \\
\hline & Galaxolide (HHCB) & 46 & 25 & 20 & 5 \\
\hline & Tonalide (AHTN) & 58 & 25 & 20 & 10 \\
\hline & Musk ketone (MK) & 56 & 20 & 25 & 25 \\
\hline \multirow[t]{9}{*}{$\begin{array}{l}\text { Sludge } \\
\mu \mathrm{gg} / \mathrm{kg} \text { wet weight }\end{array}$} & Cashmeran (DPMI) & & 17 & 9 & 3 \\
\hline & Celestolide ADBI) & & 22 & 9 & 3 \\
\hline & Phantolide (AHDI) & & 22 & 9 & 3 \\
\hline & $\begin{array}{l}\text { Musk ambrette } \\
(\mathrm{MA})\end{array}$ & & 35 & 30 & 10 \\
\hline & Traseolide (ATII) & & 24 & 9 & 3 \\
\hline & Musk xylene (MX) & & 69 & 30 & 10 \\
\hline & Galaxolide (HHCB) & & 26 & 9 & 3 \\
\hline & Tonalide (AHTN) & & 25 & 15 & 5 \\
\hline & Musk ketone (MK) & & 39 & 45 & 15 \\
\hline $\begin{array}{l}\text { Blue mussels } \\
\text { ng/g lipid }\end{array}$ & Cashmeran (DPMI) & 23 & & 260 & 85 \\
\hline \multirow[t]{8}{*}{$\begin{array}{l}\text { Average lipid } \\
\text { content } 1.3 \% \\
\end{array}$} & Celestolide ADBI) & 75 & & 260 & 85 \\
\hline & Phantolide (AHDI) & 27 & & 260 & 85 \\
\hline & $\begin{array}{l}\text { Musk ambrette } \\
\text { (MA) }\end{array}$ & 31 & & 525 & 175 \\
\hline & Traseolide (ATII) & 73 & & 260 & 85 \\
\hline & Musk xylene (MX) & 31 & & 525 & 175 \\
\hline & Galaxolide (HHCB) & 35 & & 260 & 85 \\
\hline & Tonalide (AHTN) & 21 & & 525 & 175 \\
\hline & Musk ketone (MK) & 37 & & 1310 & 435 \\
\hline
\end{tabular}


Table 5.1 Continued

\begin{tabular}{|l|l|c|r|r|r|}
\hline $\begin{array}{l}\text { Fox liver } \\
\text { ng/g lipid }\end{array}$ & Cashmeran DPMI) & 85 & & 1155 & 385 \\
\hline $\begin{array}{l}\text { Average lipid } \\
\text { content } 7.2 \%\end{array}$ & Celestolide ADBI) & 110 & & 1155 & 385 \\
\hline & Phantolide (AHDI) & 45 & & 1155 & 385 \\
\hline & $\begin{array}{l}\text { Musk ambrette } \\
\text { (MA) }\end{array}$ & 30 & & 2315 & 770 \\
\hline & Traseolide (ATI) & 250 & & 1155 & 385 \\
\hline & Musk xylene (MX) & 35 & & 2315 & 770 \\
\hline & Galaxolide (HHCB) & 70 & & 1155 & 385 \\
\hline & Tonalide (AHTN) & 30 & & 2315 & 770 \\
\hline & Musk ketone (MK) & 35 & & 5785 & 1930 \\
\hline
\end{tabular}

Recovery of Traseolide above $200 \%$ is due to matrix interference which has a high impact at the very low concentration in the spiked samples.

Measured concentrations of musk compounds in the various matrices are displayed in Table 5.2 to Table 5.5. 


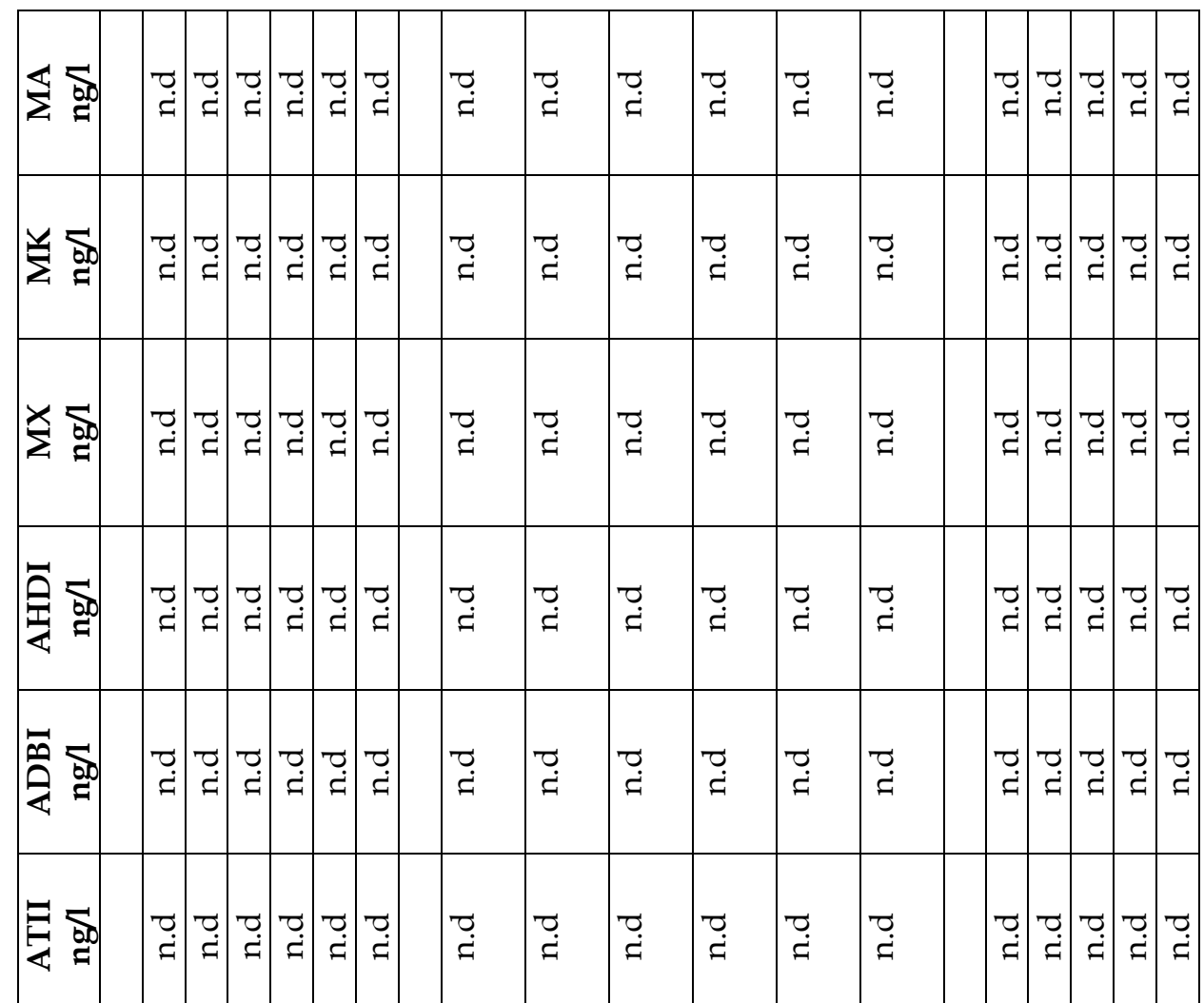

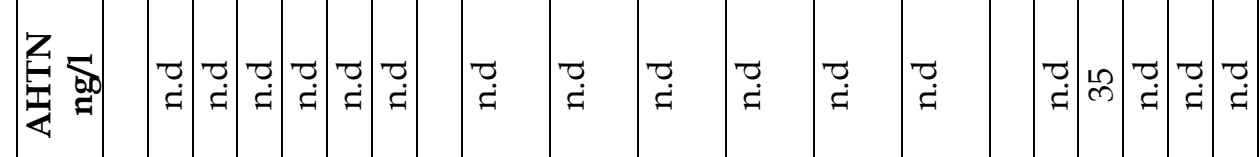

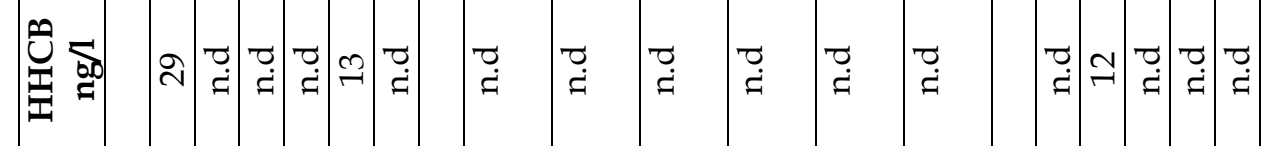

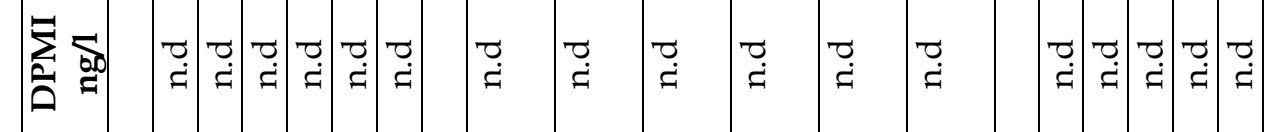

\begin{tabular}{|c|c|c|c|c|c|c|c|c|c|c|c|c|c|c|c|c|}
\hline & 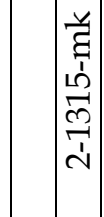 & 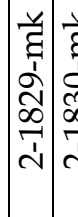 & 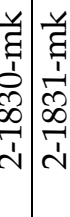 & 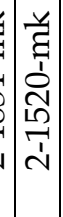 & 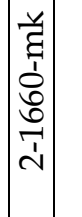 & 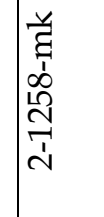 & 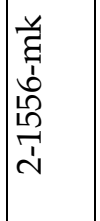 & 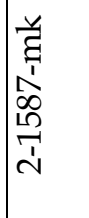 & 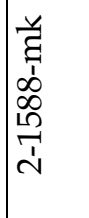 & 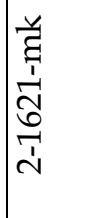 & 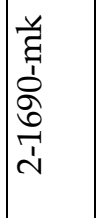 & & 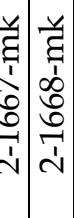 & 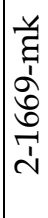 & 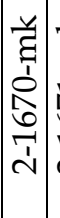 & \\
\hline 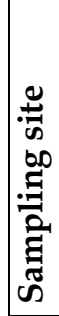 & 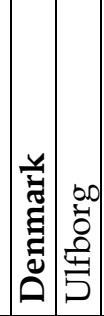 & 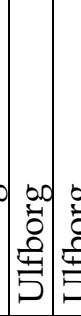 & 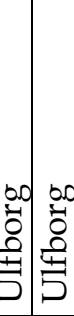 & 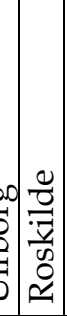 & 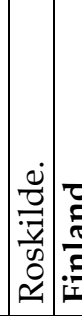 & 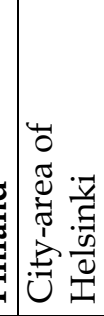 & 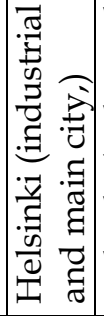 & 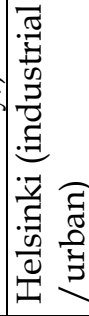 & 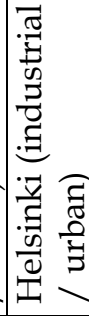 & 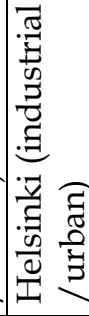 & 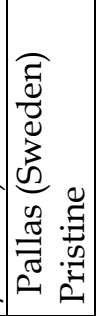 & 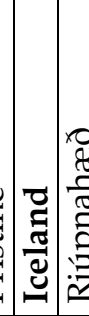 & 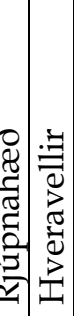 & 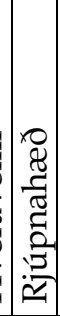 & 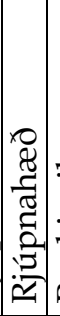 & \\
\hline
\end{tabular}




\begin{tabular}{|c|c|c|c|c|c|c|c|c|c|c|}
\hline $\begin{array}{l}4 \\
\sum\end{array}$ & ت゙્ & $\ddot{\ddot{E}}$ & ¿̈ં & $\ddot{\check{\epsilon}}$ & تॄ̈ & $\vec{i}$ & تُ & $\ddot{\check{E}}$ & ¿ே. & ¿্் \\
\hline & $\ddot{\check{I}}$ & $\ddot{\check{I}}$ & $\ddot{\check{I}}$ & $\ddot{\check{\Xi}}$ & $\ddot{\check{g}}$ & 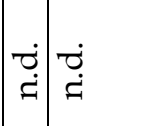 & تே் & ت் & 苂 & $\ddot{\check{\Xi}}$ \\
\hline 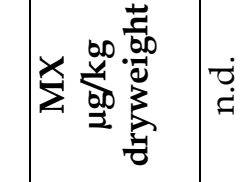 & ت்̇ & 元 & $\ddot{\ddot{E}}$ & $\ddot{\mathscr{E}}$ & $\ddot{\ddot{I}}$ & |̇ं: & تே் & ت் & تே & ت્ّ \\
\hline & in & 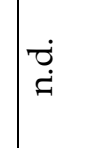 & $\ddot{\check{\epsilon}}$ & $\ddot{\check{\epsilon}}$ & تே̈ & $\approx$ & से & $\ddot{\ddot{\Xi}}$ & \% & ت્ટ \\
\hline & $\stackrel{8}{\circ}$ & $\ddot{\ddot{g}}$ & $\ddot{\check{g}}$ & $\stackrel{102}{\leftrightarrows}$ & $\stackrel{\overbrace{}}{\circ}$ & ¿ृ & 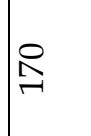 & के & in & 舫 \\
\hline & 吕 & $\mid \ddot{\nexists}$ & $\ddot{\check{g}}$ & $\stackrel{\varpi}{\sharp}$ & تே̈ & o & పే & $\tilde{\widetilde{\pi}}$ & $\nexists \tilde{z}$ & 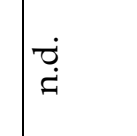 \\
\hline & 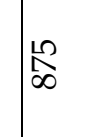 & 粕 & 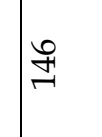 & $\infty$ & $\stackrel{\infty}{\rightleftarrows}$ & 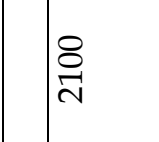 & 总 & 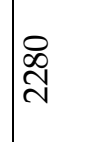 & E & $\infty$ \\
\hline 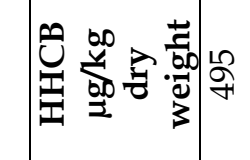 & : & d & 总 & $\stackrel{\infty}{a}$ & $\widehat{\mathscr{q}}$ & 离 & 通 & 总 & হ & 苦 \\
\hline 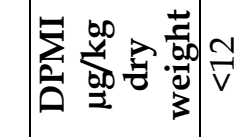 & 7 & $\mid \ddot{\nexists}$ & تृ & $\ddot{\ddot{a}}$ & تேं & ¿্் & تُ & đి & $\stackrel{8}{\circ}$ & ¿ே. \\
\hline 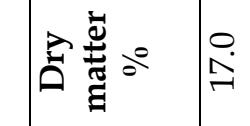 & ia & $\stackrel{2}{\circ}$ & đå & के & 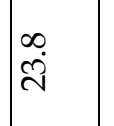 & Ȧ & 苑 & 芦 & $\overrightarrow{\tilde{i}}$ & 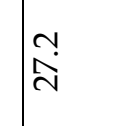 \\
\hline 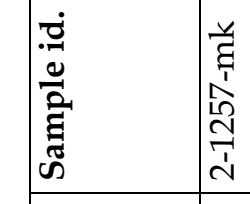 & 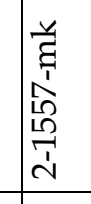 & 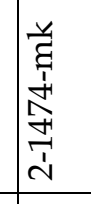 & 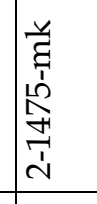 & 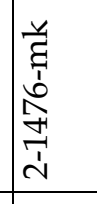 & 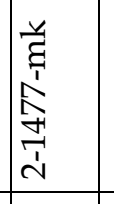 & 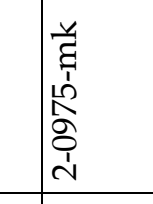 & 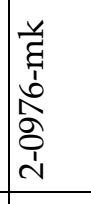 & 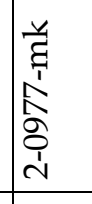 & 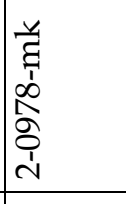 & 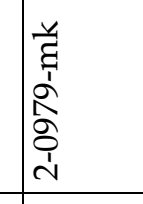 \\
\hline $\bar{a}$ & & 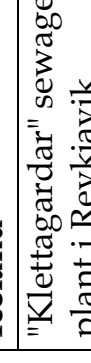 & 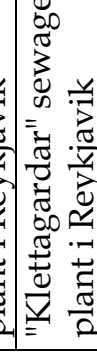 & 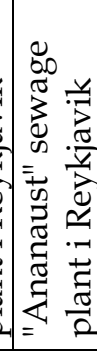 & 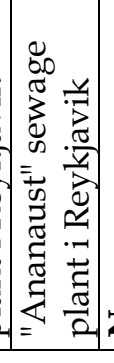 & 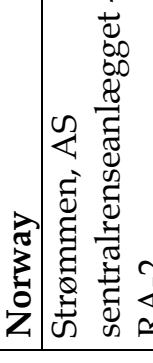 & 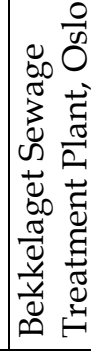 & & 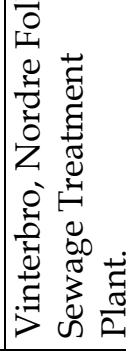 & 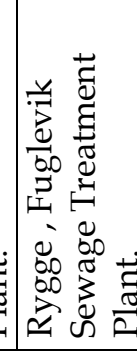 \\
\hline
\end{tabular}




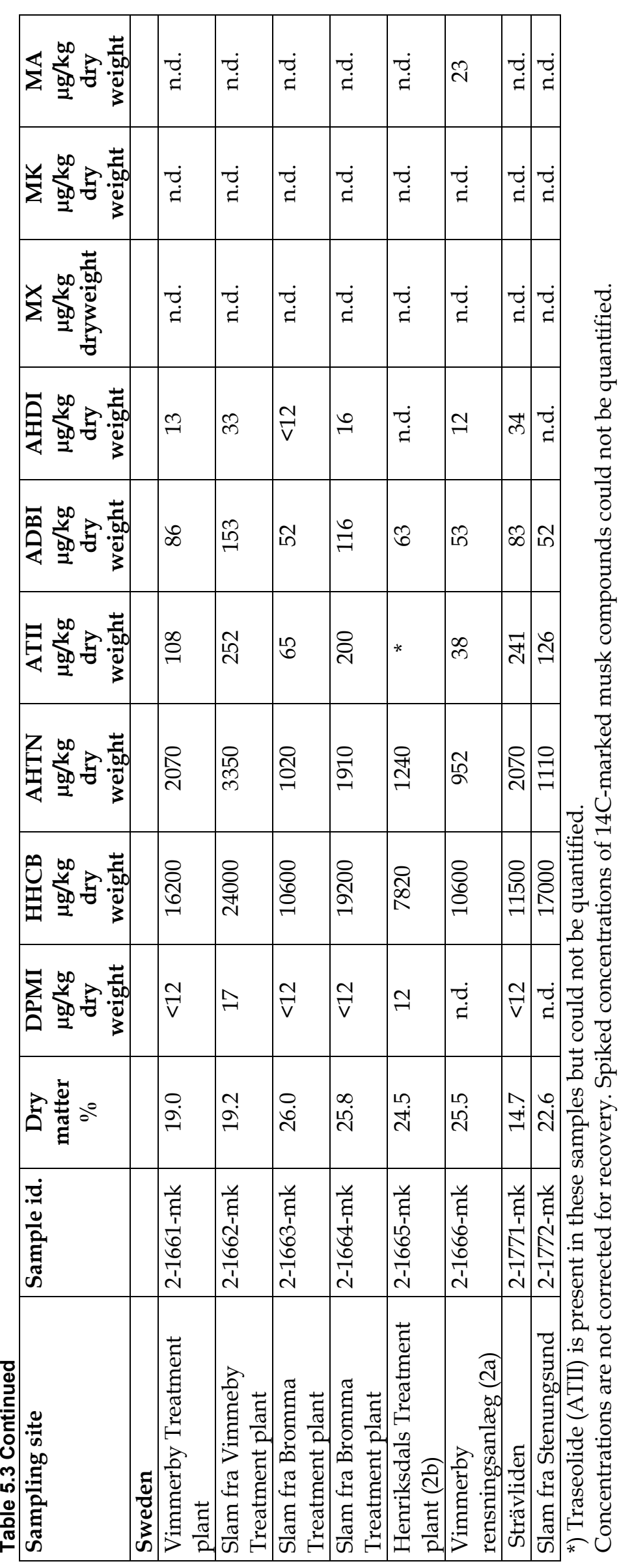




\begin{tabular}{|c|c|c|c|c|c|c|c|c|c|}
\hline 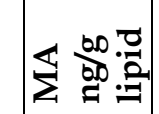 & $\vec{a}:$ & 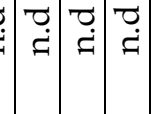 & $\ddot{\check{E}}$ & $\ddot{\pi}$ & $\overrightarrow{\dot{A}}$ & $\underset{\check{I}}{\tilde{g}}$ & ت્ & تָ & $\ddot{\check{g}}$ \\
\hline 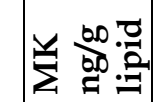 & 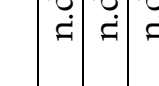 & : & $\ddot{\pi}$ & $\ddot{\pi}$ & $\stackrel{T}{\dot{x}}$ & $\ddot{d}$ & $\widetilde{\Xi}$ & $\tilde{j}$ & 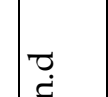 \\
\hline 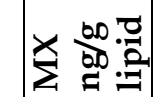 & 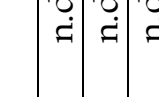 & $=$ & $\ddot{\square}$ & $\ddot{\pi}$ & $\stackrel{T}{\dot{x}}$ & $\widetilde{\Xi}$ & İ & $\bar{\pi}$ & $\overparen{E}$ \\
\hline$=$ & & & $\ddot{E}$ & $\ddot{x}$ & $\ddot{\dot{x}}$ & Ż & $\overparen{g}$ & $\vec{J}$ & $\overparen{\nearrow}$ \\
\hline 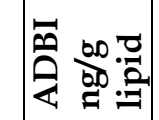 & 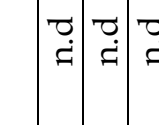 & $\tau$ & $\check{\check{I}}$ & Tَ̈ & 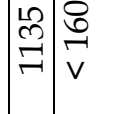 & $\tilde{E}$ & สิ & i & 各 \\
\hline 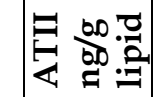 & & & $\ddot{\sharp}$ & $\overparen{\pi}$ & 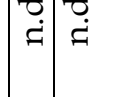 & ت્ּ & $\widetilde{\Xi}$ & $\overparen{T}$ & $\approx$ \\
\hline 0 & 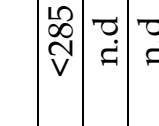 & $\begin{array}{lll}\tau \\
= \\
=\end{array}$ & $\check{\check{I}}$ & 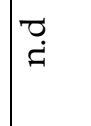 & 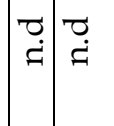 & $\vec{Z}$ & $\overparen{E}$ & 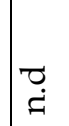 & $\vec{v}$ \\
\hline 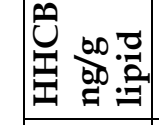 & $\widetilde{\Xi}$ & 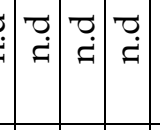 & $\check{\check{E}}$ & 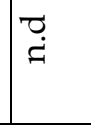 & 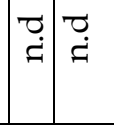 & $\overparen{\check{I}}$ & تॄ & $\vec{J}$ & $\overparen{\Xi}$ \\
\hline 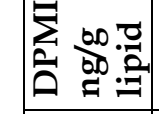 & : & $\vec{\Xi}: \vec{E}$ & 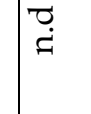 & $\ddot{\pi}$ & $\overrightarrow{\dot{A}}$ & כָ & ت̈ & $\vec{T}$ & $\overparen{E}$ \\
\hline 童: & + & $\left\{\begin{array}{l}n \\
0\end{array}\right.$ & $\stackrel{\oplus}{\rightarrow}$ & ְi & ণ্่ & 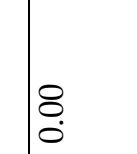 & กี & I. & ปี \\
\hline 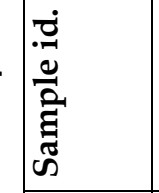 & 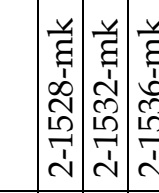 & 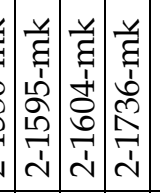 & 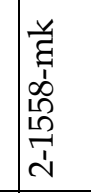 & 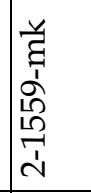 & 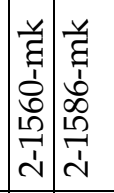 & 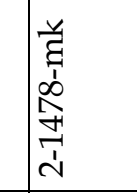 & & 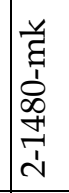 & 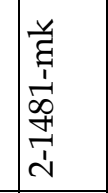 \\
\hline 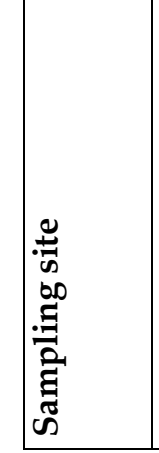 & 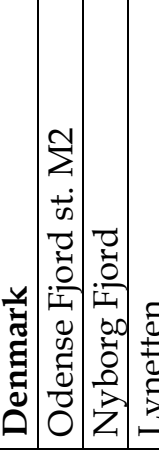 & 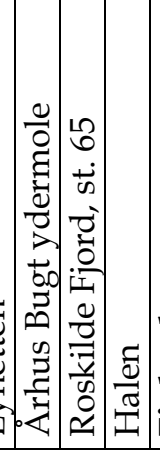 & 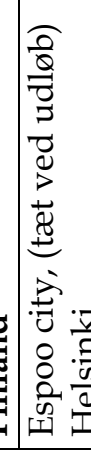 & 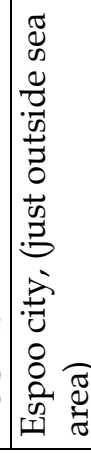 & 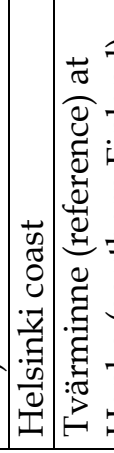 & 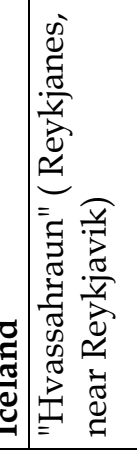 & 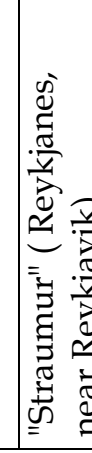 & 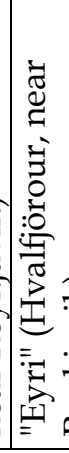 & 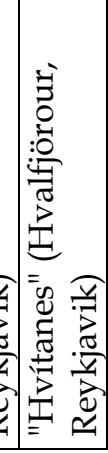 \\
\hline
\end{tabular}




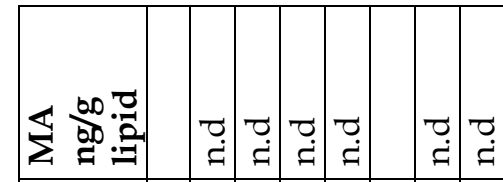

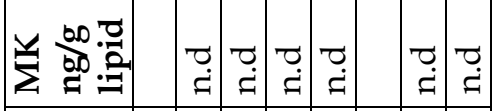

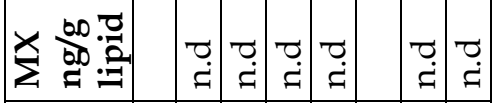

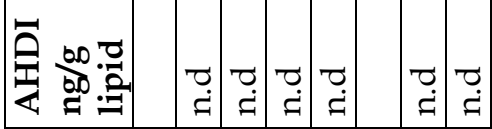

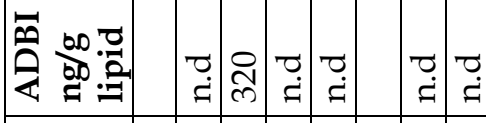

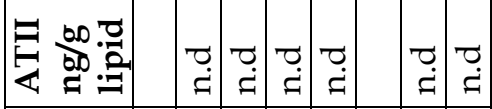

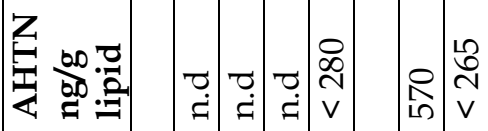

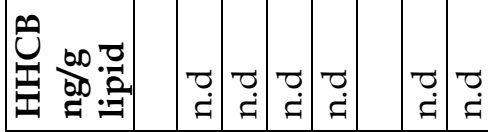

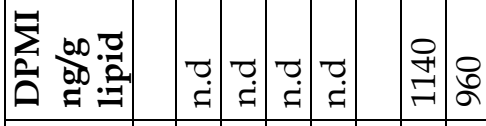
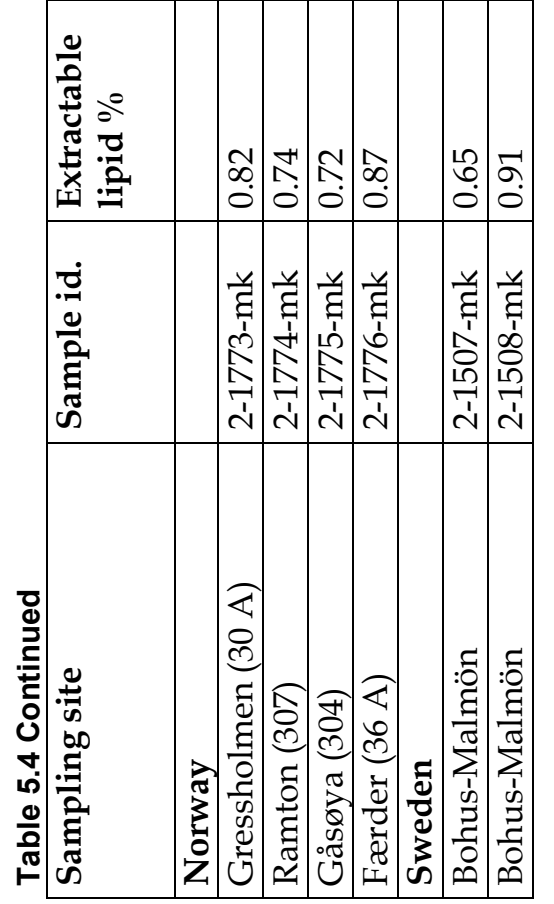

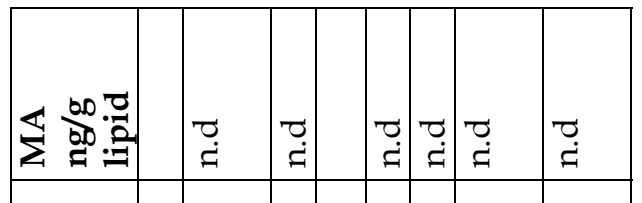

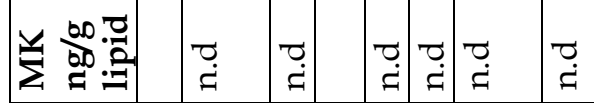

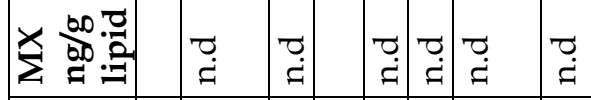

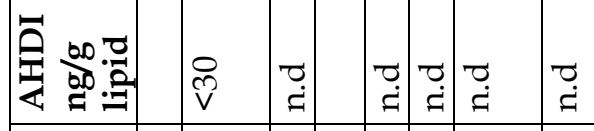

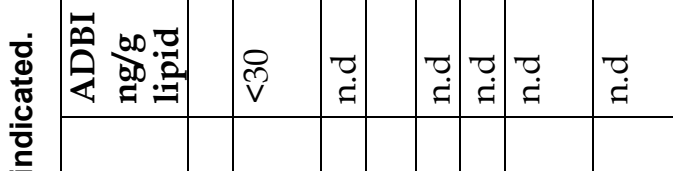

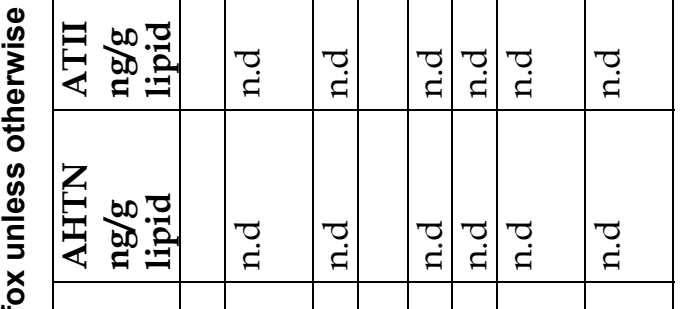

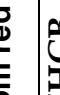
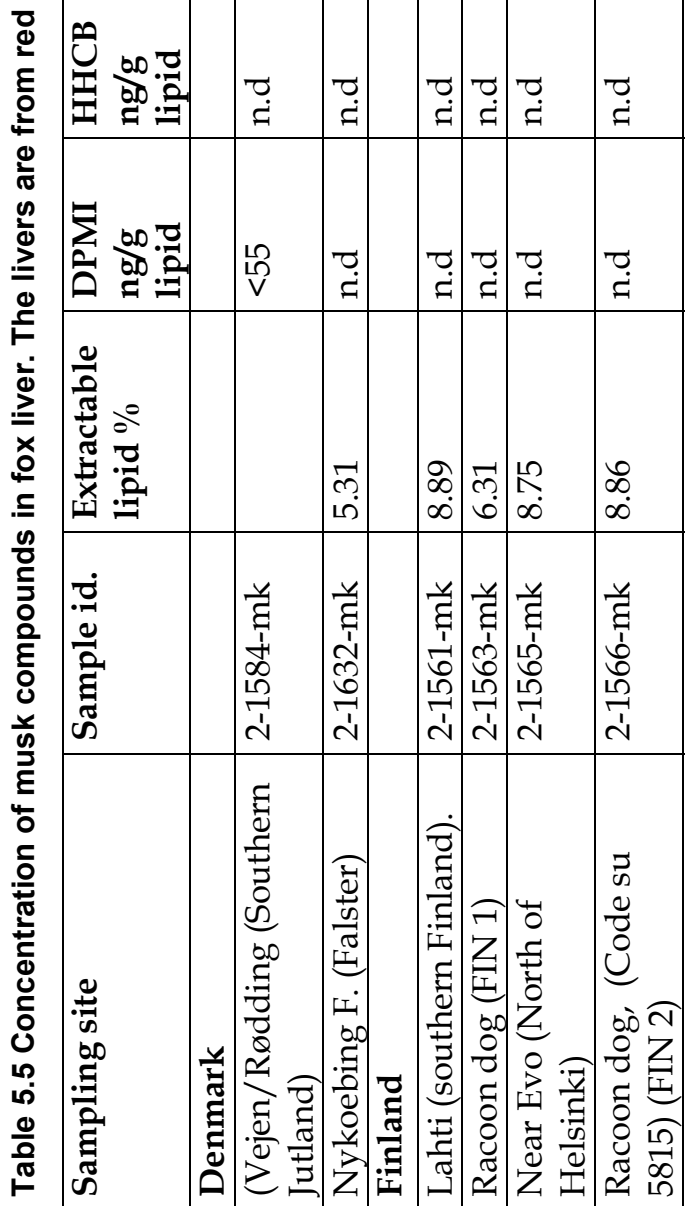


\begin{tabular}{|c|c|c|c|c|c|c|c|}
\hline 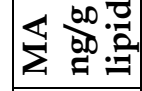 & $\overparen{\Xi}$ & $\overparen{I}$ & $\vec{T}$ & $\ddot{\Xi}$ & $\widetilde{\Xi}$ & $\overparen{\Xi}$ & $\begin{array}{l}\tau \\
\dot{\Xi}\end{array}$ \\
\hline 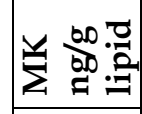 & $\overparen{\nexists}$ & ت्ञ & $\ddot{E}$ & $\ddot{\Xi}$ & $\widetilde{\Xi}$ & $\ddot{ت}$ & $\widetilde{Z}=\vec{\tau}$ \\
\hline 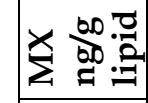 & A & ت & $\ddot{I}$ & $\ddot{g}$ & $\overparen{\Xi}$ & $\ddot{\check{g}}$ & 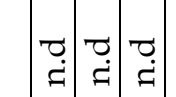 \\
\hline 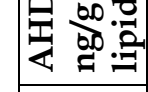 & تَّ & 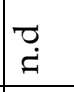 & $\overrightarrow{\check{\pi}}$ & تृ & $\vec{v}$ & $\overrightarrow{\breve{H}}$ & : \\
\hline 8 & m & ت্ت & $\ddot{\pi}$ & 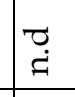 & 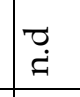 & $\ddot{\check{E}}$ & 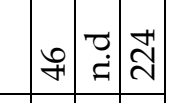 \\
\hline 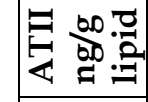 & 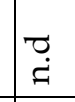 & ت्ञ & $\ddot{E}$ & $\ddot{\widetilde{E}}$ & బ్లై & $\vec{E}$ & 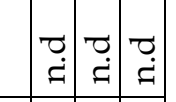 \\
\hline & $\widetilde{E}$ & שֶ. & $\widetilde{E}$ & $\ddot{E}$ & $\overparen{\Xi}$ & $\ddot{\Xi}$ & \\
\hline a. & 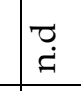 & 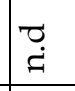 & $\vec{\rightleftarrows}$ & تृ & $\overparen{\Xi}$ & $\ddot{\rightleftarrows}$ & 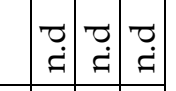 \\
\hline 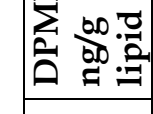 & $\vec{g}$ & $\widetilde{\pi}$ & $\widetilde{\tau}$ & $\overparen{\check{g}}$ & $\breve{\Xi}$ & $\stackrel{\breve{G}}{\check{E}}$ & $\ddot{\vec{A}}$ \\
\hline 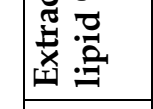 & 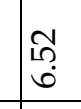 & 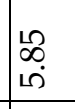 & \begin{tabular}{l}
$\infty$ \\
0 \\
0 \\
\hdashline \\
\hdashline
\end{tabular} & 忿 & 勧 & $\begin{array}{l}\vec{D} \\
\text { in } \\
\end{array}$ & 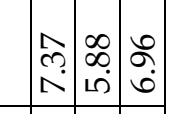 \\
\hline 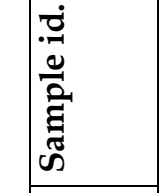 & 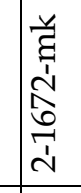 & 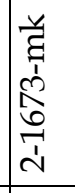 & 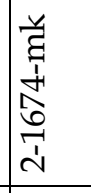 & 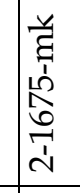 & 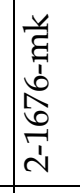 & 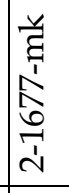 & 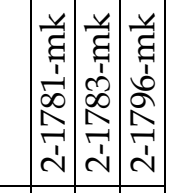 \\
\hline 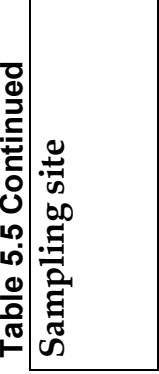 & 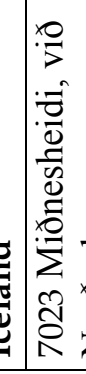 & $\mid$ & 焉 & בֶ. & Fis & 言 & 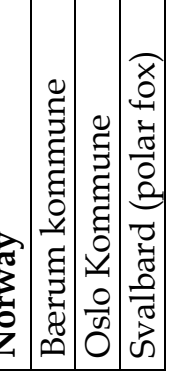 \\
\hline
\end{tabular}


The results reported in Tables 5.2 -5.5 are not corrected for recovery. It is not possible to give exact recovery percent for the musk compounds from mussels and liver samples as 5 out of six experiments (liver and mussels) to determine recovery did not show any recovery. The possible reason for this may be that the samples were spiked at a too low level to estimate recovery. Nominal concentration in the final extract was $5 \mathrm{ng} / \mathrm{ml}$ for fox liver and $20 \mathrm{ng} / \mathrm{ml}$ for blue mussels. Further, the GPC method used for clean up might have been unstable. Due to time pressure the experiments could not be repeated. Thus, the contents of musk compounds reported in Table 5.4 - Table 5.5 should be considered as the minimum concentrations in the analysed samples. Sediment samples were spiked with deuterium marked musk xylene and Tonalide but the compounds could not be quantified in the extracts. However, Peak areas in the chromatograms show high variation. Especially, the 4 samples from Iceland, the sample from the industrial area of Espo in Finland and from Strømmen sentralrensningsanlæg in Norway show low recovery. 


\section{Discussion and conclusion}

In 2002 the Nordic Environmental Monitoring group (NTEM) initiated a screening project supported by the NMR to generate information about the occurrence and distribution of musk compounds in the Nordic environment. The NTEM group was responsible for collection of samples that were transported to NERI in Denmark and analysed for content of 9 synthetic musk compounds.

Sample types included rain water, sewage sludge, blue mussels and fox liver. Information from literature on findings in other studies are summarized in a separate chapter.

Polycyclic musks are detected more frequently and at higher concentrations than the nitro musk compounds. This is in good accordance with the use pattern of these compounds with polycyclic musks replacing nitro musks as there has been some concern about possible adverse effects of these compounds to the consumer.

The analytical methods are more sensitive for the polycyclic musks with higher recovery and lower detection limit. This may be another reason for the more frequent findings of polycyclic musks.

Musk compounds are generally not detected in rainwater. Two samples from Denmark, one from Sweden and one from Iceland contain Galaxolide (HCCB) and the Icelandic sample contained Tonalide (AHTN) as well. No pattern in the occurrence can be concluded from these findings.

Sewage sludge shows the highest frequency of findings. All sewage sludge samples contain polycyclic musk compounds and usually a mixture of all 6 compounds. This confirms the suggestion by EU 2002 and EU 2003 that musk compounds are removed from sewage water primarily by adsorption to sludge particles. Galaxolide and Tonalide are present at much higher concentrations than the other compounds. Galaxolide concentrations range within $250-26500 \mu \mathrm{g} / \mathrm{kg}$ dry weigt compared to $70-3600 \mu \mathrm{g} / \mathrm{kg}$ dry weight of Tonalide. There is high variation, more than 1 order of magnitude, of recovery between sludge extracts. It is therefore not meaningful to compare concentrations at different sites. The big variation in concentration of different musk compounds in the extracts made it necessary to dilute the extracts several times to measure all the polycyclic musk compounds. This has further contributed to the overall uncertainty of the results. The results should be considered as minimum values. Polycyclic musks are used in many consumer products like cosmetics and cleaning products and are therefore likely to end up in the sewer system. Even though most of the musk compounds may be adsorbed to sludge, effluents from sewage treatment plants are an important source of musk compounds in the aquatic environment. This is reflected in Gaterman et al 1999 who found high concentrations of musk compounds in eels from lakes with high input of effluents from sewage treatment plants compared to those in lakes with low input of effluents Gaterman et al 1999). If sludge from sewage treatment plants are spread on agricultural land soil organisms will become exposed to musk compounds at a $\mathrm{mg} / \mathrm{kg}$ level.

Gaterman et al 2002 have demonstrated that the bioaccumulation of musk compounds in freshwater fish and mussels is dependent on the fat content of the animal tissue as well as on the metabolism of these compounds by various species. An additional obvious factor is also the concentration of the musk compounds in the water. Thus, the animals 
including mussels in pond water receiving effluents from sewage plants have been found to contain significant amounts of musk compounds, while the content of these compounds in animals in sea water has been found to be none to relatively low (Fromme et al 2001, Gaterman et al. 1999). In the present study polycyclic musks have been detected in 11 mussel samples out of 20 distributed all over the Nordic countries. Cashmeran was detected in two samples, Tonalide in 6 samples, Phantolide in one sample at concentration levels up to $960 \mathrm{ng} / \mathrm{g}$ extractable liquid. Celestolide was detected in 6 samples showing the highest concentrations, one sample from Iceland containing $11500 \mathrm{ng} / \mathrm{g}$ extractable liquid. The low content of lipid in mussels results in high uncertainty in the results. Galaxolide, the most frequently found musk compounds in the sewage sludge, was not detected in any of the samples. Galaxolide was found in mussels from ponds receiving effluents from sewage plants (Gaterman 1999). The absence of galaxolide in samples from the present study may be explained on the basis of the factors (discussed above), which influence bioaccumulation of musk compounds in mussels having less fat content. However, it can not be ruled out that the absence of galaxolide in any of the mussel samples may be associated with a discrepency of the GPC method used for clean up.

Five out of the 15 investigated fox livers were found to contain musk compounds. The type and amount of musk compounds present in these samples were very different. Celestolide was detected in four samples with the highest concentration, $224 \mathrm{ng} / \mathrm{g}$ extractable liquid in a sample from the arctic area of Svalbard. Traseolide was detected in one sample, Phantolide in two samples and musk xylene in one sample. The variation may be expected as the native places and thus the food available to these animals also varies. There are no data available in the literature to compare our findings with any similar study. It should be noted that galaxolide was not found in any of the liver samples.

The absence or low frequency of musk xylene, musk keton and musk ambrette in the environment may be explained by a limited use of these compounds in the consumer products. Musk ambrette is not permitted in cosmetic products due to its phototoxic or neurotoxic properties (EU Cosmetic Directive ). The Cosmetic Directive has recently regulated the use of musk xylene and musk ketone (maximum 1.0\% MX and $1.4 \% \mathrm{MK}$ in fine perfumes) in cosmetic products (26th approximation of the Cosmetic Directive, 15 April 2002). Safety evaluation of MX and MK in EU (by ECB) is not yet complete.

The results of the present study are in agreement with the data in literature, which show that sewage sludge contain significant amounts of polycyclic musks. Although the toxic effects of these compounds at the levels found in the environment have not yet been demonstrated, these compounds may bioaccumulate. In the recently adopted opinions, EU Scientific Committee on Cosmetic Products and the Non-Food Products (SCCNFP) has found the use of $2,4 \%$ and $0,96 \%$ of galaxolide and tonalide, respectively, -in eau du toilette safe for consumers. However, the SCCNFP did not consider the environmental impact of these compounds.

Most of the studies, so far, assessing environmental burden of musk compounds have concentrated on nitromusks, galaxolide and tonalide. In the present study, all of the important industrial musk compounds were included. The study has revealed that celestolide is also very frequently found in the environment, and the concentration of this compound in the environment is comparable to those of tonalide and galaxolide. To reduce the burden of the nordic environments with musk compounds following steps may be required: Specific regulations concerning emissions, updating sewage plants to 
efficiently degrade the musk compounds, and monitoring of musk compound in the environment, effluents and sewage sludge in particular. Monitoring of musk compounds in aquatic fauna is also relevant and should be focused on tissue with high lipid content. In rain water galaxolide and tonalide should serve as indicator compounds in monitoring programmes 


\section{References}

Balk, F., Ford, R.A., 1999, Environmental risk assessment for the polycyclic musks AHTN and HHCB in the EU. I. Fate and exposure assessment. Toxicology Letters 111, 57-79.

Carlsson, G., Örn, S., Andersson, P.L., Söderström, H., Norrgren, L. 2000, The impact of musk ketone on reproduction in zebrafish (Danio rerio). Marine Environmental Research 50, 237-241.

Eisenhardt, S., Runnebaum, B. Bauer, K., Gerhard, I. 2001, Nitromusk compounds in women with gynecological and endocrine dysfunction. Environmental Research Section A $87,123-130$.

Eriksson, S., Darnerud, P.O., Aune, M., Bjerselius, R., Slanina, P., Cnattingius, S., Glynn, A. 2003. Syntetiska myskföreningar i bröstmjölk och fisk. Resultatrapport till Naturvårdsverkets Mijöövervakningseneht. Avtalsnummer 219021, 37 pp.

\section{EU Cosmetic Directive 76/768/EEC}

EU 2002: Risk assessment musk ketone, Environment only. R321 205 env. Rapporteur: the Ministry of Housing, Spatial Planning and the Environment in consultation with the Ministry of Social affairs and Employment and the Ministry of Public Health, Wellfare and Sport. 49 pp. Draft May 2002

EU 2003: Risk assessment musk xylene, Environment only. R322 301 env. Rapporteur: the Ministry of Housing, Spatial Planning and the Environment in consultation with the Ministry of Social affairs and Employment and the Ministry of Public Health, Wellfare and Sport. 52 pp. Draft January 2003

Fromme, H., Otto, T., Pilz, K. 2001, Polycyclic musk fragrances in different environmental compartments in Berlin (Germany). Water Research 35, 121-128.

Gatermann, R., Hellou, J., Hühnerfuss, H., Rimkus, G., Zitko, V. 1999, Polycyclic and nitromusks in the environment: A comparison between Canadian and European aquatic biota. Chemosphere 38, 3431-3441.

Gatermann, R., Biselli, S., Hühnerfuss, H., Rimkus, G:G:, Hecker, M., Karbe, L. 2002. Synthetic musks in the environment. Part 1: Species-dependent bioaccumulation of polycyclic and nitro musk fragances in freshwater fish and mussels. Archives of Environmental Contamination and Toxicology 42, 437-446.

Glesne, Ola, Norwegian Pollution Control Authority. 2003. Personal Communication.

Hajslova, J. 2002, Determination of alkylphenols and musk compounds in waste water, sediments and fish from Sweden. Unpublished report. 
Herren, D., Berset, J.D. 2000, Nitro musks, nitro musk amino metabolites and polycyclic musks in sewage sludges. Quantitative determination by HRGC-ion trap$\mathrm{MS} / \mathrm{MS}$ and mass spectral characterization of the amino metabolites. Chemosphere 40, 565-574.

Käfferlein, H.U., Angerer, J. 2001, Trends in the musk xylene concentrations in plasma samples from the general population from 1992/1993 to 1998 and the relevance of dermal uptake. International Archive of Occupational Environmental Health 74, 470476.

Kallenborn, R., Gatermann, R., Rimkus, G.G. 1999, Synthetic musks in environmental samples: indicator compounds with relevant properties for environmental monitoring, Viewpoint, Jounal of Environmental Monitoring, 1, 70N-74N.

Kallenborn, R., Gatermann, R., Nygård, T. Knutzen, J., Schlabach, M. 2002???, Synthetic Musks in Norwegian marine fish samples collected in the vicinity of densely populated areas, SPØRG OLA

Nylander, A. 2001. Syntetiska myskföreningar, En riskbedömning. Projektrapport från utbildningen i Ekotoxikologi, Ekotoxikologiska avdelingen, Nr. 77, Uppsala Universitet, $63 \mathrm{pp}$.

Ott, M., Failing, K., Lang, U., Schubring, C., Gent, H-J., Georgii, S., Bruun, H. 1999, Contamination of human milk in middle Hesse, Germanny - A cross-sectional study on the changing levels of chlorinated pesticides, PCB congeners and recent levels of nitro musks, Chemospere 38, 13-32.

Pedersen, K.H., 2000. Indhold af syntetiske moskusforbindelser i dambrugsfisk og modermælk fra Danmanrk. Spialeafhandling i miljøkemi ved Københavns Universitet. Pp 118.

Rimkus, G.G. 1998, Synthetic musk fragrances in human fat and their potential uptake. In Frosch, P.J., Johansen, J.D., White, I.R. (eds.) Fragrances. Beneficial and adverse effects. ISBN 3-540-81871-6, Springer Verlag, pp 136-149.

Rimkus, G.G., 1999, Polycyclic musk fragrances in the aquatic environment. Toxicology Letters, 111, 37-56.

Rimkus, G.G., Gatermann, R., Hühnerfuss, H. 1999, Musk xylene and musk ketone amino metabolites in the aquatic environment. Toxicology Letters 111, 5-15.

Simonich, S.L., Begley, W.M., Debaere,G., Eckhoff, W.S., 2000, Trace Analysis of Fragrance Materials in Wastewater and treated wastewater. Environmental Science and Technology, 34, 959-965.

Suter-Eichenberger, R., Altorfer, H., Lichtensteiger, W., Schlumpf, M. 1998, Bioaccumulation of musk xylene (MX) in developing and adult rats of both sexes.

Chemosphere 36, 2747-2762. 
Winkler, M., Kopf, G., Hauptvogel, C., Neu, T. 1998, Fate of artificial musk fragrances associated with suspended particulate matter (SPM) from the River Elbe (Germany) in comparison to other organic contaminants. Chemosphere 37, 1139-1156. 


\section{Appendix 1}

Manual for sample handling and protocol of sampling for the project: "Analysis of Musk compounds in a Nordic cooperation on screening".

\section{Sample types:}

The project includes analysis of musk compounds in the following sample types (matrices):

A: Precipitation (rain) - to be taken during July - September

B: Municipal sewage sludge - to be taken as soon as possible (July -August)

C: Blue Mussel (Mytilus edulis) - to be taken October

D: Fox liver - to be taken as soon as possible (Latest end of September)

\section{General remarks:}

Musk compounds are widely used in cosmetics (incl. soaps) and a row of technical products, so great care has to be taken to avoid contamination of the samples.

When handling the samples gloves are mandatory, and measures should be taken to exclude that the personnel involved are using cosmetics (especially soap, perfume and deodorants) containing musk compounds in the sample-handling period. Even cleaned laboratory air can contain vapors of musk compounds; so prolonged direct contact with the laboratory air should be avoided.

Furthermore all utensils coming in contact with the samples should be solvent rinsed with 3 times acetone and 3 times n-pentane following the normal cleaning. Glass and metal utensils should finally be heated for 2 hours at $450{ }^{\circ} \mathrm{C}$; Teflon utensils should be heated for 12 hours at $200{ }^{\circ} \mathrm{C}$.

Contact with polymer utensils should be kept at a minimum, and restricted to utensils made of Teflon and Nylon, the latter only in form of special sample bags as Rilsan ${ }^{\circledR}$ bags.

All samples of one kind from each country should be sent together to NERI as soon as possible after the sampling. NERI should be notified about the arrival of samples in order to ensure that the samples are received and handled properly.

\section{A: Precipitation}

Five rain water samples should be collected from at least three stations representing different levels of expected emissions of musk substances, e.g. industrial area (especially if containing industries fabricating or using musk substances) or city-areas, suburban area, rural area.

The sampling should preferably take place during a period of warm weather over a period of max one month, covering precipitation of at least $20 \mathrm{~mm}$. The sampling should preferably take place during periods of heavy rain.

The samples should preferable be taken with a cooled wet-only sampler (e.g. of a type like NSA 181/KE, G. K. Walter Eigenbrodt Environmental Measuring Systems, Königsmoor, Germany), with a funnel area of about $500 \mathrm{~cm}^{2}$. Otherwise the sampling laboratory has to insure that the sample temperature does not exceed $4{ }^{\circ} \mathrm{C}$, that the sample is kept protected against UV-light and that no dry deposition takes place. 
The final sampling size shall be 2 - 3 liter, corresponding to about 40-60 mm of precipitation, and shall be transported to the analytical laboratory preferable within 24 hours kept at a temperature between $0{ }^{\circ} \mathrm{C}$ and $4{ }^{\circ} \mathrm{C}$ together with the following information:

Information to be gathered and delivered together with the sample:

Position: Geographical coordinates, height above ground level, description of immediate surroundings, e.g. pine forest, street, open land etc.

Sampling area type: Industrial, city, suburban area, rural area.

Possible emitters of musk substances in the area

Sampling period

Weather condition during the sampling and the week before

Sampling method including description of the sampling device.

Other remarks

\section{B: Municipal sewage sludge}

Five samples of municipal sewage sludge should be collected from at least three stations representing different levels of expected emissions of musk substances, e.g. industrial area (especially if containing industries fabricating or using musk substances and emitting waste water to the sewage plant) or city-areas, suburban area, rural area. The sludge collected has to be fresh from the sewage plant, taken within one hour from final dewatering/stabilization, following a period of normal weather conditions.

Approximately 500 gram of sludge shall be collected in Rilsan bags or rinsed glass jars, and placed at $4{ }^{\circ} \mathrm{C}$ in the dark immediately.

The sample shall be transported to the analytical laboratory preferably within 24 hours at a temperature between $0^{\circ} \mathrm{C}$ and $4^{\circ} \mathrm{C}$ together with the following information: Information to be gathered and delivered together with the sample:

Type of wastewater catchment area: Industrial, municipal or mixed wastewater Possible emitters of musk substances in the area

Sampling date

Information about the plant (type, size, running conditions at the sampling time etc, any abnormalities in the operation of the plant during the week before sampling.)

Weather condition during the sampling and the week before

Other remarks

\section{C: Blue Mussels}

Four samples of Blue Mussels (Mytilus edulis) should be collected from at least three stations representing different levels of expected emissions of musk substances, e.g. industrial area (especially if containing industries fabricating or using musk) or cityarea, suburban area, rural area.

The mussel samples are collected as $30-40$ preferably bottom-dwelling individuals at $40-60 \mathrm{~mm}$ lengths, collected by hand or trawl after the spawning season (in October).

The living mussels are rinsed for sand etc. at the shells with water from the sampling place, and depurated for 24 hours in the same type of water before dissection. After opening and passive dewatering for 10 seconds, all the soft tissue (incl. the adductor muscle) is removed and weighted for each individual and the length of the shell measured.

The soft tissue (incl. the adductor muscle) from all the mussels are pooled in a glass jar, and frozen at $-20^{\circ} \mathrm{C}$. 
The sample shall be transported to the analytical laboratory preferably within 24 hours at a temperature between $-5{ }^{\circ} \mathrm{C}$ and $-20{ }^{\circ} \mathrm{C}$ together with the following information: Information to be gathered and delivered together with the sample:

Position: Geographical coordinates, description of sampling area including water depth and type of bottom.

Possible emitters of musk substances in the area

Sampling date and method

General information concerns the conditions of the mussels (fouling, physical state etc.).

Other remarks

\section{D: Fox (liver)}

Four samples of Fox (Vulpes vulpes) liver should be collected from stations representing different levels of expected emissions of musk substances, e.g. industrial area (especially if containing industries fabricating or using musk) or city-area, suburban area, rural agriculture area and intended pristine nature area.

The fox liver samples shall consist of whole livers and lower jaws from two males of at least two years of age collected before the beginning of the autumn shedding to winter fur, preferable before the end of September.

The foxes are to be newly killed and dissected within 24 hours after the killing. The whole livers are placed in double Rilsan or Teflon bags, while the bags for the lower jaws can be of ordinary plastic.

The samples from the two individuals shall be kept separate, and frozen at $-20{ }^{\circ} \mathrm{C}$ within 4 hours from dissection.

The sample shall be transported to the analytical laboratory as soon as possible at a temperature between $-5{ }^{\circ} \mathrm{C}$ and $-20^{\circ} \mathrm{C}$ together with the following information:

Information to be gathered and delivered together with the sample:

Position: Geographical coordinates, description of sampling area in general terms including a possible quote of the feeding habits of the actual foxes

Possible emitters of musk substances in the area

Sampling date and method

General information concerns the conditions of the foxes (sex, nutritional and physical state, a rough estimate of age etc.).

Other remarks 


\section{Appendix 2}

\section{Information on sampling stations}

\section{Denmark}

The location of the Danish sampling sites and geographical coordinates and classification of the sites in relation to pollution can be found in table 1 . The number of specimen collected from different sites is summarized in table 2. Even those sites, where collection did not succeed, are listed in table 2. Figure 1 displays the Danish sampling sites.

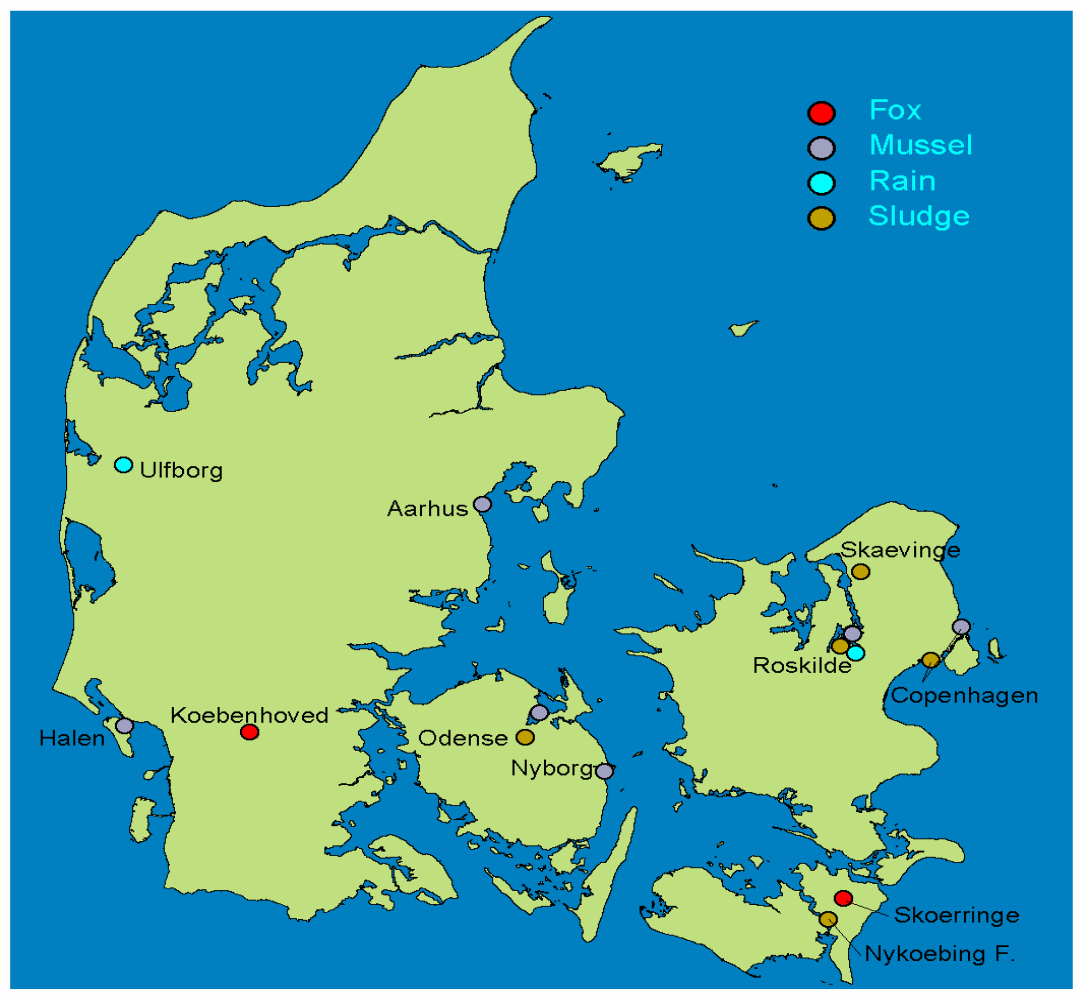

Figure 1. Danish sampling sites. 
TABLE 1. Coordinates of the sampling sites in Demark year 2002. Joint Nordic screening project on musk substances. National Environment Research Institute (NERI).

\begin{tabular}{|l|l|l|l|}
\hline Roskilde & 55.65283 & 12.04097 & Urban + industrial \\
\hline Roskilde (marine area) & 55.70043 & 12.06707 & \\
\hline Copenhagen & 55.36291 & 12.27023 & Urban + industrial \\
\hline Halen & 55.42572 & 12.36518 & \\
\hline Skaevinge & 55.26251 & 08.26334 & Pristine \\
\hline Nykoebing F. & 55.54265 & 12.07465 & Rural \\
\hline Skoerringe & 54.46264 & 11.52552 & Suburban/rural \\
\hline Odense & 54.50365 & 11.57401 & Forest \\
\hline Odense (marine area) & 55.23561 & 10.25167 & Urban + industrial \\
\hline Nyborg & 55.28458 & 10.29574 & \\
\hline Koebenhoved & 55.16566 & 10.48277 & Urban + industrial \\
\hline Ulfborg & 55.25197 & 09.03546 & Forest \\
\hline Aarhus & 56.17380 & 08.25205 & Forest \\
\hline
\end{tabular}

TABLE 2.Summary of the samples collected at the sampling sites in Denmark 2002. Joint Nordic screening project on musk substances. National Environment Research Institute (NERI).

\begin{tabular}{|c|c|c|c|c|}
\hline Specimen & $\begin{array}{l}\text { Total } \\
\text { number of } \\
\text { samples }\end{array}$ & $\begin{array}{l}\text { Urban+Industrial } \\
\text { sites (n) }\end{array}$ & $\begin{array}{l}\text { Forest+rural } \\
\text { sites (n) }\end{array}$ & $\begin{array}{l}\text { Pristine } \\
\text { sites (n) }\end{array}$ \\
\hline Sludge & 5 & $\begin{array}{l}\text { Copenhagen (1) } \\
\text { Nykoebing F. (1) } \\
\text { Roskilde (1) } \\
\text { Odense (1) }\end{array}$ & Skaevinge (1) & \\
\hline Rain water & 5 & $\begin{array}{l}\text { Roskilde }(2) \\
\text { Copenhagen }(0)\end{array}$ & Ulfborg (3) & \\
\hline Blue mussel & 6 & $\begin{array}{l}\text { Copenhagen (1) } \\
\text { Odense (1) } \\
\text { Aarhus (1) }\end{array}$ & $\begin{array}{l}\text { Roskilde (1) } \\
\text { Nyborg (1) }\end{array}$ & Halen (1) \\
\hline Red fox & 2 & Copenhagen (0) & $\begin{array}{l}\text { Skoerringe (1) } \\
\text { Koebenhoved (1) }\end{array}$ & Ulfborg (0) \\
\hline
\end{tabular}




\section{Sludge sampling}

Sampling was performed according to the method description of NERI Appendix 1. The samples were collected by NERI. All single samples are summarized in table 3 .

TABLE 3. Sludge samples collected by Denmark 2002. Joint Nordic screening project on musk substances. National Environmental Research Institute (NERI).

\begin{tabular}{|l|l|l|l|}
\hline Sample & Site/sewage plant & $\begin{array}{l}\text { Person equivalent } \\
\text { (peq) }\end{array}$ & Classification \\
\hline Sample 1. & Skaevinge & $5000-6000$ & rural municipal \\
\hline Sample 2. & Roskilde & 50000 & $\begin{array}{l}\text { mainly municipal } \\
\text { less industry }\end{array}$ \\
\hline Sample 3. & Avedøre & 400000 & heavy industrial+municipal \\
\hline Sample 4. & Nykoebing F. & 40000 & $\begin{array}{l}\text { industrial+municipal } \\
\text { mixed }\end{array}$ \\
\hline Sample 5. & Odense NE & 80000 & $\begin{array}{l}\text { municipal + industrial } \\
\text { mixed }\end{array}$ \\
\hline
\end{tabular}

\section{Rain water sampling}

Three samples of rainwater were collected in the urban area of Roskilde (at midZeeland) at the ground of a sewage plant (sample $3+4$ at one location, sample $5100 \mathrm{~m}$ apart), and two samples were collected from a coniferous forest of pristine nature in western Jutland (Ulfborg). A planned sampling in the city of Copenhagen was cancelled for logistic reasons. The samplings were performed according to the method description of NERI Appendix 1 during september-november 2002. All samples are listed in table 4.

TABLE 4. Samples of rainwater collected in Denmark 2002. Joint Nordic screening project on musk substances. National Environmental Research Institute (NERI).

\begin{tabular}{|l|l|l|l|}
\hline Sample & Site & Sampling period & Volume (L) \\
\hline Sample 1. & Ulfborg & $13.9 .2002-20.10 .2002$ & 2 liter \\
\hline Sample 2. & Ulfborg & $20.10 .2002-15.11 .2002$ & 2 liter \\
\hline Sample 3. & Roskilde & $26.9 .2002-7.10 .2002$ & 2 liter \\
\hline Sample 4. & Roskilde & $7.10 .2002-1.11 .2002$ & 2 liter \\
\hline Sample 5. & Roskilde & $2.11 .2002-15.11 .2002$ & 2 liter \\
\hline
\end{tabular}

\section{Blue mussel sampling}

Sampling of mussels was performed by the personnel from the different counties in connection with the sampling for the National Monitoring Programme at stations at Zealand, Funen and Jutland in October 2002. The mussels were dissected according to the method description given by NERI (ref: NERI-DK 28.6.2002). The soft tissue from at least 20 mussel individuals was kept frozen in brown glass jars until analysis. All samples are summarised in table 5. 
TABLE 5. Blue mussel samples collected in the coastal sea area of Denmark 2002. Joint Nordic screening project on musk substances. National Environmental Research Institute (NERI).

\begin{tabular}{|c|c|c|c|c|}
\hline Sample & Date & $\begin{array}{l}\text { Site } \\
\text { (name, depth in m) }\end{array}$ & $\begin{array}{l}\text { Size class }(\mathrm{mm}) ; \\
\text { mean }\end{array}$ & Remarks \\
\hline Sample 1. & $\begin{array}{l}\text { October, } \\
2002\end{array}$ & $\begin{array}{l}\text { Copenhagen } \\
\text { (Lynetten, 5) }\end{array}$ & $40-60 ; 48$ & $\begin{array}{l}\text { In vicinity of } \\
\text { effluent outlet }\end{array}$ \\
\hline Sample 2. & $\begin{array}{l}\text { October, } \\
2002\end{array}$ & $\begin{array}{l}\text { Roskilde } \\
\text { (Roskilde Fjord, 2) }\end{array}$ & $40-60 ; 42$ & Rather unpolluted area \\
\hline Sample 3. & $\begin{array}{l}\text { October, } \\
2002\end{array}$ & $\begin{array}{l}\text { Nyborg } \\
\text { (Nyborg Fjord, 3) }\end{array}$ & $40-60 ; 54$ & Industrial polluted \\
\hline Sample 4. & $\begin{array}{l}\text { October, } \\
2002\end{array}$ & $\begin{array}{l}\text { Wadden Sea } \\
(\text { Halen, } 1)\end{array}$ & $40-60 ; 54$ & Reference sea area \\
\hline Sample 5. & $\begin{array}{l}\text { October, } \\
2002\end{array}$ & $\begin{array}{l}\text { Odense } \\
\text { (Odense Fjord, 4) }\end{array}$ & $40-60 ; 49$ & $\begin{array}{l}\text { In vicinity of } \\
\text { effluent outlet }\end{array}$ \\
\hline Sample 6. & $\begin{array}{l}\text { October, } \\
2002\end{array}$ & $\begin{array}{l}\text { Aarhus } \\
\text { (Aarhus Harbour, 6) }\end{array}$ & $40-60 ; 52$ & $\begin{array}{l}\text { Polluted industrial } \\
\text { harbour area }\end{array}$ \\
\hline
\end{tabular}

\section{Fox samples}

The samples of liver tissue were collected from red fox (Vulpes vulpes). Only two animals could be sampled during the autumn 2002, and the planned collection of fox samples from urban/industrial location did not succeed. The summary of the samples with background information is presented in table 6 .

TABLE 6. The samples of red fox and raccoon dog collected in Denmark 2002. Joint Nordic screening project on musk substances. National Environmental Research Institute (NERI).

\begin{tabular}{|l|l|l|l|l|l|}
\hline Sample & Animal & Sex & Hunting date & $\begin{array}{l}\text { Age } \\
\text { (year) }\end{array}$ & Site and classification \\
\hline Sample 1. & Red fox & $\mathrm{F}$ & 25.10 .2002 & $<2$ & $\begin{array}{l}\text { Koebenhoved } \\
\text { Forest in rural area }\end{array}$ \\
\hline Sample 2. & Red fox & $\mathrm{F}$ & 10.11 .2002 & $>2$ & $\begin{array}{l}\text { Skoerringe } \\
\text { Forest in suburban area }\end{array}$ \\
\hline
\end{tabular}

\section{Finland}

The location of the Finnish sampling sites is presented in the figure 2 and geographical coordinates and classification of the sites in relation to pollution can be found in table 7 Number of specimen collected from different sites is summarized in table 8 .

Even those sites, where collection and hunting of animals didn't result any success, are pointed out in table 7 and figure 2 . 
TABLE 7 Coordinates of the sampling sites in Finland year 2002. Joint Nordic screening project on musk substances. Finnish Environment Institute (SYKE).

\begin{tabular}{|l|c|c|l|}
\hline Sampling site & North & East & Site classification \\
\hline Helsinki & 60,2795 & 24,8735 & Urban + industrial \\
Helsinki (sea area) & 60,1072 & 25,0326 & \\
\hline Espoo & 60,1158 & 24,7484 & Urban + industrial \\
\hline Porvoo & 60,3774 & 25,6142 & Urban + industrial \\
\hline Pornainen & 60,4608 & 25,3989 & Rural / pristine \\
\hline Jokela & 60,5525 & 24,9543 & Rural \\
\hline Evo / Lahti & 61,1954 & 25,1568 & Suburban \\
\hline Evo & 61,1954 & 25,1568 & Forest / pristine /South \\
\hline Pallas & 67,9980 & 24,1724 & Forest / pristine /North \\
\hline Kevo & 69,6594 & 27,0519 & Pristine / subarctic \\
\hline Tvärminne & 59,8376 & 23,2175 & Pristine / marine \\
\hline
\end{tabular}

TABLE 8 Summary of the samples collected in the sampling sites in Finland 2002. Joint Nordic screening project on musk substances. Finnish Environment Institute (SYKE).

\begin{tabular}{|l|c|l|l|l|}
\hline Specimen & $\begin{array}{c}\text { Total number of } \\
\text { samples }\end{array}$ & $\begin{array}{l}\text { Urban+Industrial } \\
\text { sites (n) }\end{array}$ & $\begin{array}{l}\text { Forest+rural sites } \\
\text { (n) }\end{array}$ & $\begin{array}{c}\text { Pristine sites } \\
\text { (n) }\end{array}$ \\
\hline Sludge & 6 & $\begin{array}{l}\text { Helsinki (2) } \\
\text { Espoo (1) } \\
\text { Porvoo (1) }\end{array}$ & $\begin{array}{l}\text { Jokela (1) } \\
\text { Pornainen (1) }\end{array}$ & Evo (0) \\
\hline Rain water & 5 & Helsinki (5) & $\begin{array}{l}\text { Pallas } \\
\text { sampling by } \\
\text { IVL Sweden) }\end{array}$ \\
\hline Blue mussel & 4 & Helsinki (3) & Tvärminne (1) \\
\hline $\begin{array}{l}\text { Red fox } \\
\text { Red fox } \\
\text { Racoon dog }\end{array}$ & 4 & Helsinki (0) & $\begin{array}{l}\text { Evo (1) } \\
\text { Evo/Lahti (1) } \\
\text { Evo (2) }\end{array}$ & Kevo (0) \\
\hline
\end{tabular}




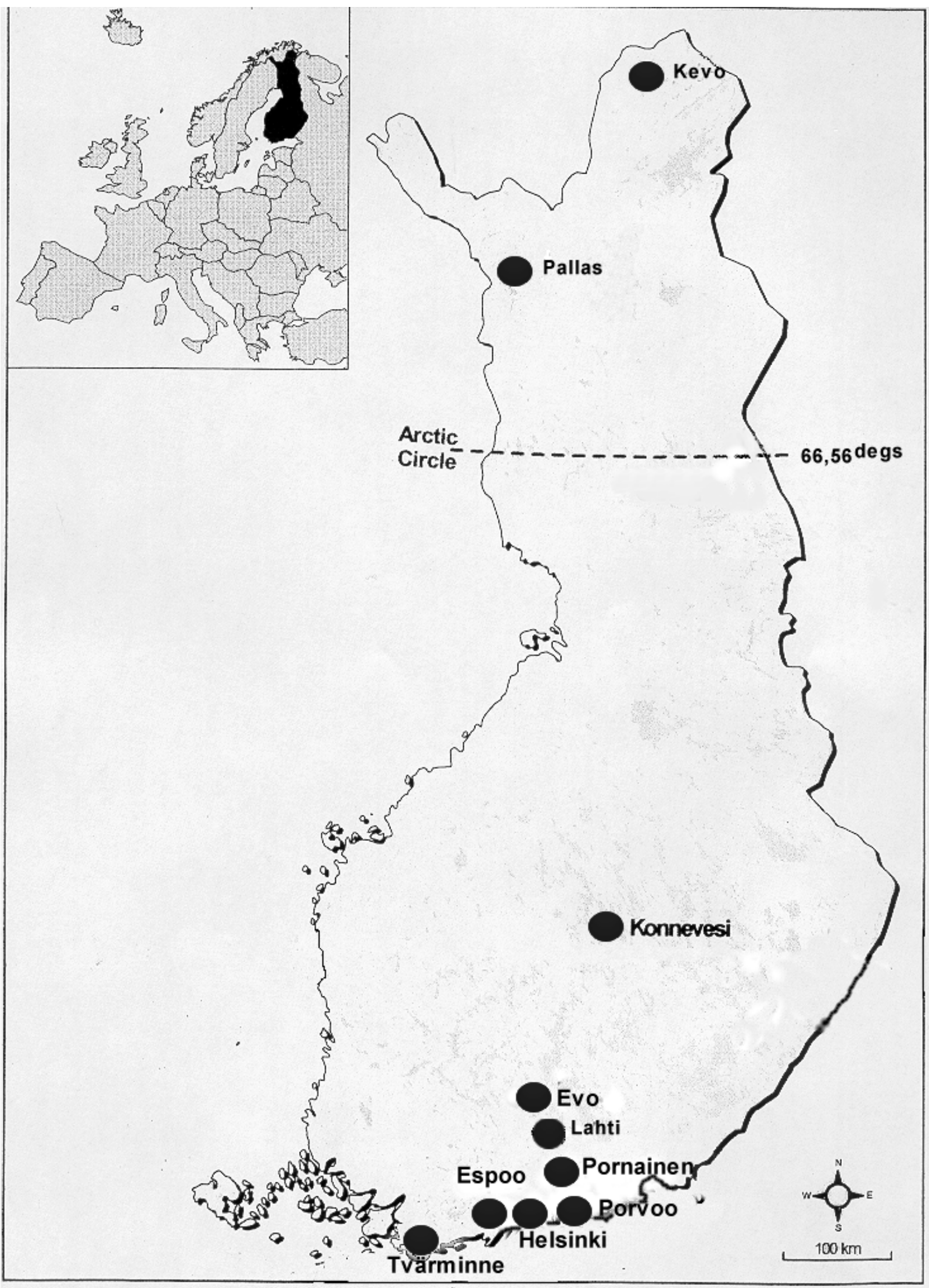

FIGURE 2. The sampling sites in Finland 2002. Joint Nordic screening project on musk substances. Finnish environment institute (SYKE). 


\section{Sludge sampling}

Sampling was performed according to the method description of NERI Appendix 1. The samples were delivered in glass jars to NERI. All single samples are summarised in table 9.

TABLE 9 Sludge samples delivered by Finland 2002. Joint Nordic screening project on musk substances. Finnish Environment Institute (SYKE).

\begin{tabular}{|l|l|c|l|l|}
\hline $\begin{array}{c}\text { Sample code } \\
\text { /date }\end{array}$ & $\begin{array}{c}\text { Site /sewage } \\
\text { plant }\end{array}$ & $\begin{array}{c}\text { Person equivalent } \\
\text { (peq) }\end{array}$ & \multicolumn{1}{|c|}{ Classification } & \multicolumn{1}{|c|}{ Remarks } \\
\hline $\begin{array}{l}\text { Sample 1. FIN } \\
3.9 .2002\end{array}$ & Jokela & $5000-6000$ & rural municipal & $\begin{array}{l}\text { Old system } \\
\text { not dewatered } \\
\text { not accepted }\end{array}$ \\
\hline $\begin{array}{l}\text { Sample 2. FIN } \\
\text { 3.9.2002 }\end{array}$ & Porvoo & $>10000$ & $\begin{array}{l}\text { industrial+municipal } \\
\text { mixed }\end{array}$ & New system \\
\hline $\begin{array}{l}\text { Sample 3. FIN } \\
\text { 3.9.2002 }\end{array}$ & Pornainen & $<2000$ & rural municipal & Old system \\
\hline $\begin{array}{l}\text { Sample 4. FIN } \\
\text { 4.9.2002 }\end{array}$ & Helsinki & $>10000$ & $\begin{array}{l}\text { industrial+municipal } \\
\text { mixed }\end{array}$ & $\begin{array}{l}\text { Largest treatment } \\
\text { plant in the North }\end{array}$ \\
\hline $\begin{array}{l}\text { Sample 5. FIN } \\
\text { 4.9.2002 }\end{array}$ & Espoo & $>10000$ & $\begin{array}{l}\text { industrial+municipal } \\
\text { mixed }\end{array}$ & $\begin{array}{l}\text { New system, } \\
\text { industrial emitter } \\
\text { in the area }\end{array}$ \\
\hline $\begin{array}{l}\text { Sample 6. FIN } \\
\text { 17.10.2002 }\end{array}$ & Helsinki & $>10000$ & $\begin{array}{l}\text { industrial+municipal } \\
\text { mixed }\end{array}$ & $\begin{array}{l}\text { Replacing sample } \\
\text { no 1 (Jokela) }\end{array}$ \\
\hline
\end{tabular}

\section{Rain water sampling}

Five samples of rainwater were collected in the industrial urban area of Helsinki. In the original plan also samples from the Evo region (southern pristine area) and the Pallas station (northern pristine area) were indented, but because of very dry summer and autumn, the samples could be collected only from the Helsinki city area. The sampler was placed in the open wood area in the vicinity of SYKE's laboratory, which is locating a little bit outside of Helsinki city. The sampler was easy to serve daily if needed. The sampling apparatus requested by NERI in their manual couldn't be used and was not available. (Cleaning of sampler, tools and bottles was made according the NERI's manual). We used a self-manufactured sampler of stainless steel plate for intensive sampling of a single rain shower (Figure 3). Sampler's size was $1 \mathrm{~m}^{2}$ and 1 $\mathrm{mm}$ rain gave 1 liter water. Sampling was started manually by opening the cover and putting 5 liters' glass bottle on its place whenever the rain began. After the rain shower, the glass bottle with rain was moved back to the cooling room $\left(+4^{\circ} \mathrm{C}\right)$ in the laboratory. That was the only way to get rainwater collected because of very dry weather during the period from 1 July to 15 October, 2002. All samples are listed in table 10 
TABLE 10.Samples of rainwater collected in Finland 2002. Joint Nordic screening project on musk substances. Finnish Environment Institute (SYKE).

\begin{tabular}{|l|l|c|c|l|}
\hline \multicolumn{1}{|c|}{ Sample code } & \multicolumn{1}{|c|}{ Site } & $\begin{array}{c}\text { Sampling } \\
\text { date/period }\end{array}$ & Volume (L) & \multicolumn{1}{|c|}{ Remarks } \\
\hline Sample 1. FIN & Helsinki & 30.8 .2002 & 2 liter & $\begin{array}{l}\text { Single heavy rain } 5 \mathrm{~mm} / \mathrm{h} \\
\text { after very long warm period }\end{array}$ \\
\hline Sample 2. FIN 2. & Helsinki & $16.9 .-15.10 .2002$ & 3 liter & $\begin{array}{l}\text { Few single rain spouts }- \\
\text { very dry }\end{array}$ \\
\hline Sample 3. FIN 3. & Helsinki & $26 .-27.10 .2002$ & 5 liter & Heavy rain $3 \mathrm{~mm} / \mathrm{h}$ \\
\hline Sample 4. FIN 4. & Helsinki & $31.10 .-1.11 .2002$ & 5 liter & Heavy rain $2 \mathrm{~mm} / \mathrm{h}$ \\
\hline Sample 5. FIN 5. & Helsinki & $9 .-10.11 .2002$ & $3-5$ liter & $\begin{array}{l}\text { Wet snow after stormy } \\
\text { weather }\end{array}$ \\
\hline
\end{tabular}

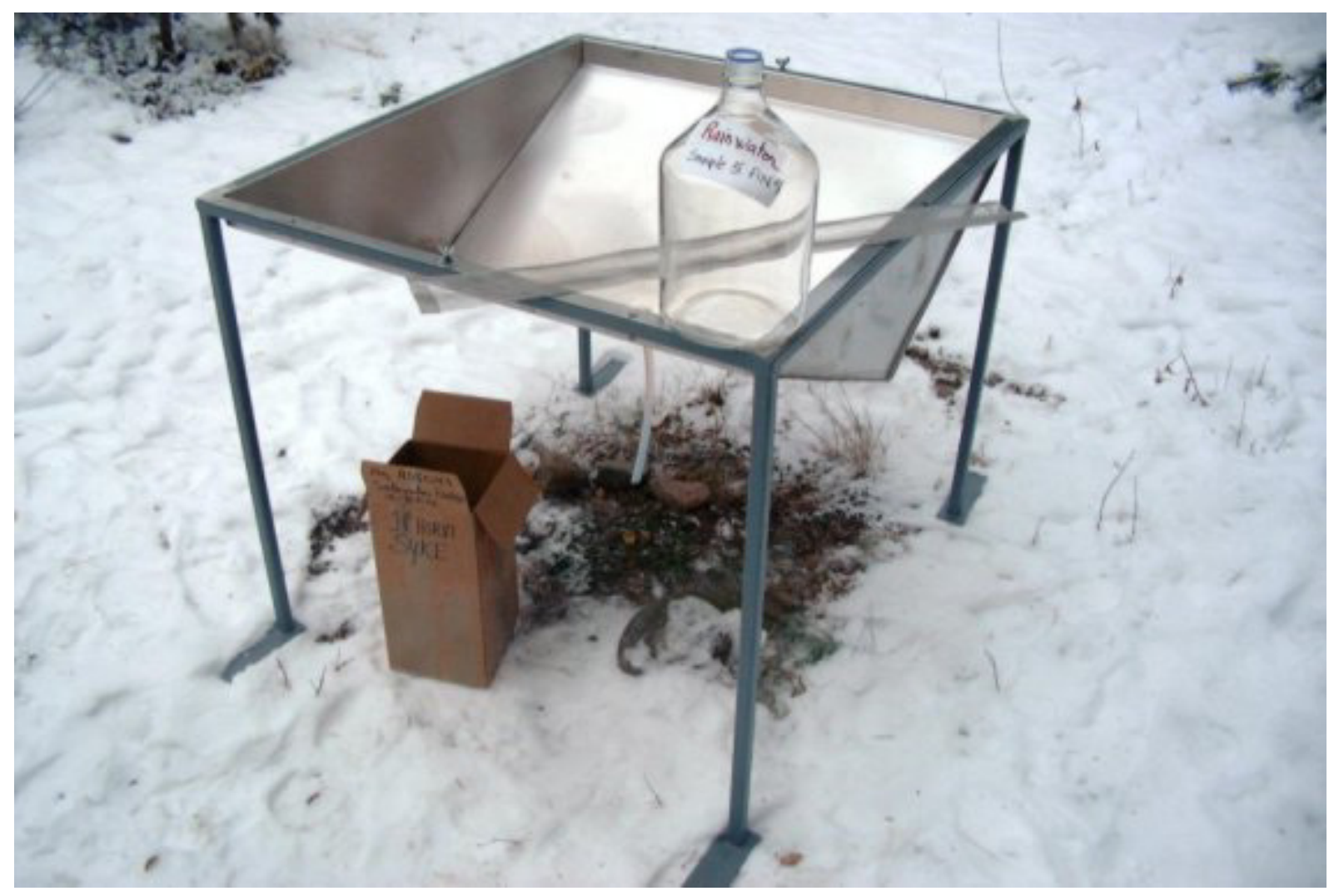

FIGURE 3. Pieces and tools for sampling of rain water in Southern Finland. Photo taken $14^{\text {th }}$ November 2002. Joint Nordic screening project on musk substances. Finnish Environment Institute (SYKE).

Figure 3. Demonstration of the sampler in use for intensive sampling of rainwater in the urban area of Helsinki, Finland. Photo taken $14^{\text {th }}$ November 2002. Joint Nordic screening project on musk substances. Finnish Environment Institute (SYKE). 


\section{Blue mussel sampling}

Sampling of mussels was performed by the divers from SYKE (Helsinki and Espoo) and from the University of Helsinki (Tvärminne, research station). The mussels were dissected according to the method description given by NERI Appendix 1. The soft tissue from more than 60 mussel individuals was removed and weighted until to $20 \mathrm{~g}$ of total sample weight reached. The samples were frozen in Rilsan bags inserted in glass jars and sent cooled to the NERI. Observe that size class requested (preferred) in the NERI's manual was as high as $40-60 \mathrm{~mm}$ lengths. Such big individuals of blue mussel cannot be found in the northern Baltic Sea. All samples are summarised in table 11

Table 11.Blue mussel samples collected in the southern coastal sea area of Finland 2002. Joint Nordic screening project on musk substances. Finnish Environment Institute (SYKE).

\begin{tabular}{|c|c|l|c|l|}
\hline Sample code & Date & Site* (depth, m) & $\begin{array}{l}\text { Size class (mm) } \\
\text { (mean } \pm \text { SD; N) }\end{array}$ & \multicolumn{1}{|c|}{ Remarks } \\
\hline Sample 1. FIN 1. & 16.10 .2002 & Espoo I (5m) & $21 \pm 4 ; 68$ & $\begin{array}{l}\text { In vicinity of } \\
\text { effluent outlet from } \\
\text { Espoo }\end{array}$ \\
\hline Sample 2. FIN 2. & 16.10 .2002 & Espoo II (5m) & $20 \pm 3,5 ; 72$ & $\begin{array}{l}\text { In vicinity of } \\
\text { effluent outlet from } \\
\text { Espoo }\end{array}$ \\
\hline Sample 3. FIN 3. & 16.10 .2002 & Helsinki (6m) & $17,5 \pm 3 ; 104$ & $\begin{array}{l}\text { In vicinity of } \\
\text { effluent outlet from } \\
\text { Helsinki }\end{array}$ \\
\hline $\begin{array}{l}\text { Sample 4. FIN 4. } \\
\text { October, 2002 }\end{array}$ & Tvärminne (8m) & $25,5 \pm 3 ; 79$ & Reference sea area \\
\hline \multicolumn{3}{|c|}{ Coastal sea sites* Espoo 1 = Longgrundet SW. Espoo 2 = Knapperskäret SW. Helsinki = } \\
Mustakupu SW, Santahamina E. Tvärminne = Brännskär (4 - 8 m).
\end{tabular}

\section{Fox and racoon dog samples}

The samples of liver tissue were collected from red fox (Vulpes vulpes) and racoon dog (Nyctereutes procyonoides, Gray). Two animals could be hunted during the autumn 2002 (fresh samples), and other two were taken from the SYKE's environment specimen bank (banked samples). The summary of the samples with background information is presented in table 12

Table 12.The samples of red fox and raccoon dog collected in Finland 2002. Joint Nordic screening project on musk substances. Finnish Environment Institute (SYKE).

\begin{tabular}{|l|l|c|l|l|c|l|}
\hline \multicolumn{1}{|c|}{ Sample code } & \multicolumn{1}{|c|}{ Animal } & Sex & \multicolumn{1}{|c|}{ Hunting date } & $\begin{array}{c}\text { Specimen to } \\
\text { NERI }\end{array}$ & $\begin{array}{c}\text { Age } \\
\text { (y) }\end{array}$ & $\begin{array}{l}\text { Site and } \\
\text { classification }\end{array}$ \\
\hline Sample 1. FIN 1. & Red fox & $\mathrm{F}$ & 5.10 .2002 & $\begin{array}{l}\text { Liver + lower } \\
\text { jaw }\end{array}$ & $>2$ & $\begin{array}{l}\text { Evo/Lahti } \\
\text { Suburban }\end{array}$ \\
\hline Sample 2. FIN 2. & Racoon dog & $\mathrm{F}$ & 10.8 .2002 & $\begin{array}{l}\text { Liver + lower } \\
\text { jaw }\end{array}$ & $>2$ & $\begin{array}{l}\text { Evo } \\
\text { pristine }\end{array}$ \\
\hline Sample 3. FIN 3. & Red fox & $\mathrm{M}$ & $\begin{array}{l}1.12 .2000 \\
\text { ESB code ke 603 }\end{array}$ & Liver & 2 & $\begin{array}{l}\text { Evo } \\
\text { pristine }\end{array}$ \\
\hline Sample 4. FIN 4. & Racoon dog & $\mathrm{M}$ & $\begin{array}{l}21.10 .2000 \\
\text { ESB code su 5815 }\end{array}$ & Liver & 2 & $\begin{array}{l}\text { Evo } \\
\text { pristine }\end{array}$ \\
\hline
\end{tabular}




\section{Iceland}

The location of the Icelandic sampling sites is presented in the figure 4 .

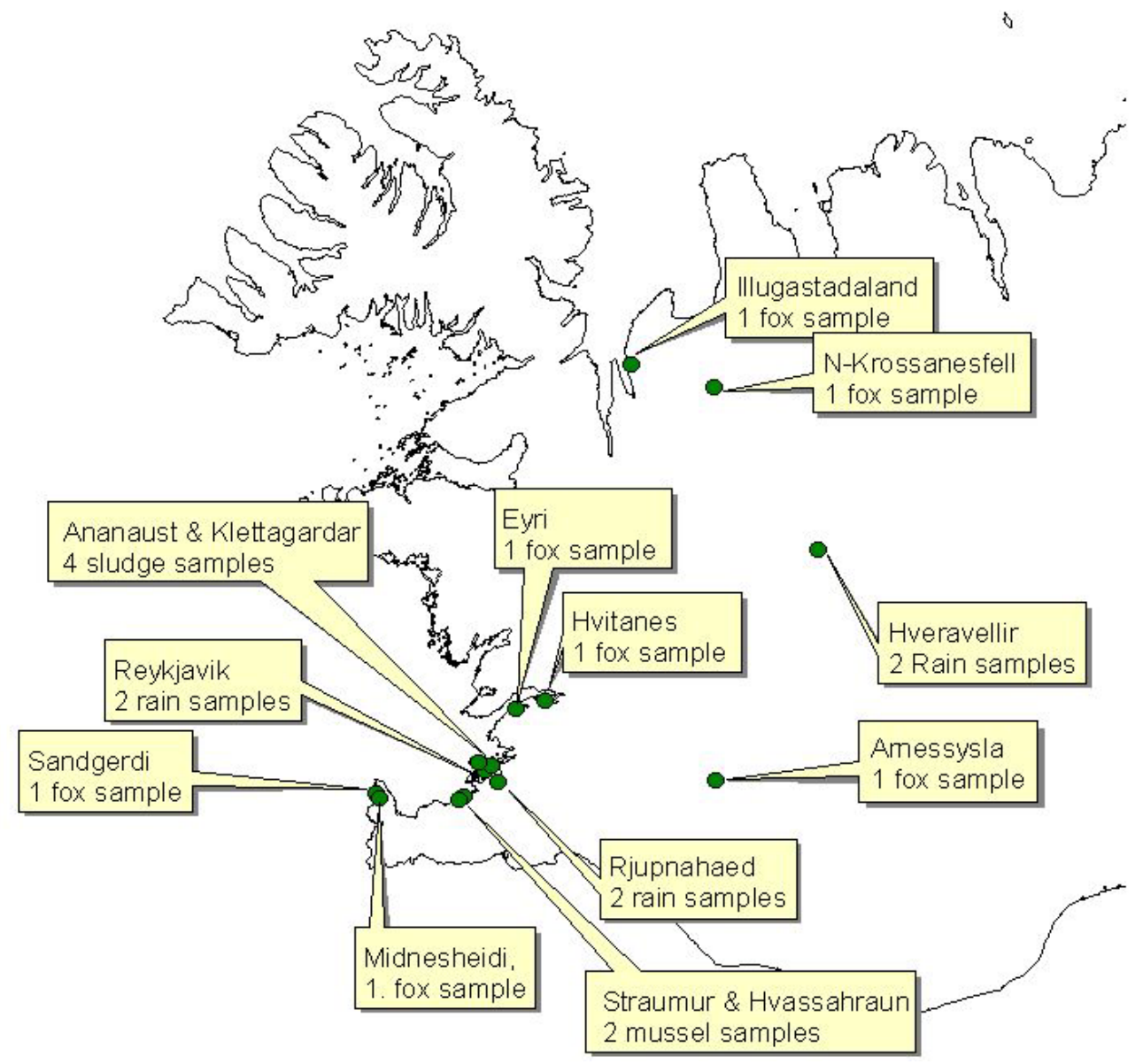

FIGURE 4. The sampling sites in Iceland 2002. Joint Nordic screening project on musk substances. Environment \& Food Agency of Iceland (UST).

\section{Sludge sampling}

Sampling was performed according to the method description of NERI Appendix 1. The samples were delivered in teflon bags (Rilsan) to NERI. See summary in table 13. 
TABLE 13.Sludge samples delivered by Iceland 2002. Joint Nordic screening project on musk substances. Environment \& Food Agency of Iceland (UST)

\begin{tabular}{|c|c|c|c|c|}
\hline $\begin{array}{l}\text { Sample code } \\
\text { /date }\end{array}$ & $\begin{array}{l}\text { Site /sewage } \\
\text { plant }\end{array}$ & $\begin{array}{l}\text { Person } \\
\text { equivalent } \\
\text { (peq) }\end{array}$ & Classification & Coordinate \\
\hline KL1. / 30.9.2002 & $\begin{array}{l}\text { Klettagardar, } \\
\text { Reykjavik }\end{array}$ & $>10000$ & $\begin{array}{l}\text { industrial }+ \text { municipal } \\
\text { mixed }\end{array}$ & $\begin{array}{l}64^{\circ} 9,122^{\prime} \mathrm{N} \\
21^{\circ} 51,994^{\prime} \mathrm{V}\end{array}$ \\
\hline KL2. / 30.9.2002 & $\begin{array}{l}\text { Klettagardar, } \\
\text { Reykjavik }\end{array}$ & $>10000$ & $\begin{array}{l}\text { industrial }+ \text { municipal } \\
\text { mixed }\end{array}$ & $\begin{array}{l}64^{\circ} 9,122^{\prime} \mathrm{N}, \\
21^{\circ} 51,994^{\prime} \mathrm{V}\end{array}$ \\
\hline AN1./ 30.9.2002 & $\begin{array}{l}\text { Ananaust, } \\
\text { Reykjavik }\end{array}$ & $>10000$ & municipal & $\begin{array}{l}64^{\circ} 9,540^{\prime} \mathrm{N},- \\
21^{\circ} 56,285^{\prime} \mathrm{V}\end{array}$ \\
\hline AN2. / 30.9.2002 & $\begin{array}{l}\text { Ananaust, } \\
\text { Reykjavik }\end{array}$ & $>10000$ & municipal & $\begin{array}{l}64^{\circ} 9,540^{\prime} \mathrm{N}, \\
21^{\circ} 56,285^{\prime} \mathrm{V}\end{array}$ \\
\hline
\end{tabular}

\section{Remarks}

Coordinate system we used is ISN93-WGS84

\section{Rain water sampling}

Five samples of rainwater were collected 1) from municipal part of Reykjavik, 2) from a suburban part of Reykjavik, and 3) from Hveravellir located in central Iceland (rural background/northern pristine area).

Sampler 1) was placed in the open area in the vicinity of the Icelandic Meteorological Institute laboratory, which is locating a within Reykjavik city.

Sampler 2) was placed in the open area on a hilltop outside of Reykjavik where the Icelandic Meteorological Institute laboratory has a full weather station.

Sampler 3) was placed in the open area in Hveravellir, central Icelandic highlands. The Icelandic Meteorological Institute also has a manned weather station in this location. All stations are served daily. The sampling apparatus requested by NERI in their manual was not available. (Cleaning of sampler, tools and bottles was made according the NERI's manual). We used a existing sampler of stainless steel plate. Sampler's size was $30 \mathrm{~cm} 2$. Sampling was continued until bottle was full. When the bottle was full of rain it was moved to a refrigerator $\left(+4^{\circ} \mathrm{C}\right)$ in the Meteorological Institute. All samples are listed in table 14 
TABLE 14 Samples of rainwater collected in Iceland 2002. Joint Nordic screening project on musk substances. Environment \& Food Agency of Iceland (UST).

\begin{tabular}{|l|l|c|c|l|}
\hline Sample code & \multicolumn{1}{|c|}{ Site } & $\begin{array}{c}\text { Sampling } \\
\text { period }\end{array}$ & $\begin{array}{c}\text { Volume } \\
\text { (L) }\end{array}$ & \multicolumn{1}{|c|}{ Remarks } \\
\hline $\begin{array}{l}\text { Reykjavik, } \\
\text { Aug. 02 }\end{array}$ & Reykjavik & Aug. 2002 & 2 liter & $\begin{array}{l}52 \mathrm{~m} \text { above sea level. Flat grassy hilltop, 100 } \\
\mathrm{m} \text { from housing }\end{array}$ \\
\hline $\begin{array}{l}\text { Reykjavik, } \\
\text { Sept. 02 }\end{array}$ & Reykjavik & Sept. 2002 & 2 liter & $\begin{array}{l}52 \mathrm{~m} \text { above sea level. Flat grassy hilltop, 100 } \\
\mathrm{m} \text { from housing }\end{array}$ \\
\hline $\begin{array}{l}\text { Hveravellir, } \\
\text { Aug. 02 }\end{array}$ & Hveravellir & Aug. 2002 & 2 liter & $\begin{array}{l}\text { 641 m above sea level. Flat, grass/lava, } \\
\text { uninhabited rural highlands }\end{array}$ \\
\hline $\begin{array}{l}\text { Rjúpnahæð, } \\
\text { Aug. 02 }\end{array}$ & Rjúpnahæð & Aug. 2002 & 2 liter & $\begin{array}{l}120 \mathrm{~m} \text { above sea level. Flat grassy hilltop, } \\
\text { near Reykajavik, Suburban }\end{array}$ \\
\hline $\begin{array}{l}\text { Rjúpnahæð, } \\
\text { Sept. 02 }\end{array}$ & Rjúpnahæð & Sept. 2002 & 2 liter & $\begin{array}{l}120 \mathrm{~m} \text { above sea level. Flat grassy hilltop, } \\
\text { near Reykajavik, Suburban }\end{array}$ \\
\hline
\end{tabular}

TABLE 15 Coordinates of rainwater sampling sites in Iceland

\begin{tabular}{|l|l|}
\hline Reykjavik & $64^{\circ} 07.646^{\prime} \mathrm{N}, 21^{\circ} 54.146^{\prime} \mathrm{V},(64.13,21.90)$ \\
\hline Hveravellir & $64^{\circ} 52.001^{\prime} \mathrm{N}, 19^{\circ} 33.723^{\prime} \mathrm{V},(64.87,19.56)$ \\
\hline Rjúpnahæð & $64^{\circ} 05^{\prime} \mathrm{N}, 21^{\circ} 51^{\prime} \mathrm{V},(64.08,21.85)$ \\
\hline
\end{tabular}

\section{Blue mussel sampling}

Sampling of mussels was performed by the Marine Research Institute of Iceland. The mussels were dissected according to the method description given by NERI (ref: NERI-DK 28.6.2002). The soft tissue from more than 30 mussel individuals was removed and weighted. The samples were frozen in Rilsan bags, and later sent cooled to the NERI.

All samples are summarised in table 15

TABLE 16 Blue mussel samples collected in the southwestern coastal sea area of Iceland 2002. Joint Nordic screening project on musk substances. Environment \& Food Agency (UST).

\begin{tabular}{|l|l|c|l|c|c|}
\hline $\begin{array}{c}\text { Sample code } \\
\text { Place }\end{array}$ & Sample date & Depth & $\begin{array}{c}\text { Coordinates } \\
\text { (degrees) }\end{array}$ & $\begin{array}{c}\text { Size class (mm) } \\
\text { (mean } \pm \text { SD; N) }\end{array}$ & $\begin{array}{c}\text { Remarks } \\
\text { Type of bottom }\end{array}$ \\
\hline Hvassahraun & 23.9 .2002 & 2 meters & $\begin{array}{l}640200- \\
220790\end{array}$ & $45 \pm 7 ; 30$ & Sand and gravel - tidal beach* \\
\hline Straumur & 23.09 .02 & 2 meters & $\begin{array}{l}640264- \\
220275\end{array}$ & $48 \pm 8 ; 31$ & Sand and gravel - tidal beach* \\
\hline Eyri & 23.09 .02 & 2 meters & $\begin{array}{l}642034- \\
214367\end{array}$ & $48 \pm 8 ; 34$ & Sand and gravel - tidal beach* \\
\hline Hvítanes & 23.09 .02 & 2 meters & $\begin{array}{l}642177- \\
212971\end{array}$ & $46 \pm 7 ; 31$ & Sand and gravel - tidal beach* \\
\hline
\end{tabular}

* Samples were collected into a bucket (w. water holes) by hand in low tide. Sampling site is on average 1,5-2 meters below sea level except on low tides, every other week. This means that on low tide it is possible to wear boots and pick up the mussels by hand. After picking the mussels were dipped into a barrel of sea water for 24 hours.

Samples were then brought to a laboratory where each individual was dissected. Dissection took ca. 1,5 hours for each sampling site, other individuals were meanwhile kept in a cooler; dissected samples were also put in cooler. After all dissection was done the samples were brought to a 20 degree cold freezer until being mailed to NERI. 
TABLE 17 Possible emitters of Musk around the blue mussel sampling sites

\begin{tabular}{|l|l|}
\hline Hvassahraun & $\begin{array}{l}\text { This site is on Reykjanes close to Reykjavik. There is an aluminum smelter \& } \\
\text { industrial activity in the area }\end{array}$ \\
\hline Straumur & $\begin{array}{l}\text { This site is on Reykjanes close to Reykjavik. There is an aluminum smelter \& } \\
\text { industrial activity in the area }\end{array}$ \\
\hline Eyri & $\begin{array}{l}\text { This site is in Hvalfjörður close to Reykjavik. There is an aluminum smelter in } \\
\text { the area }\end{array}$ \\
\hline Hvítanes & $\begin{array}{l}\text { This site is in Hvalfjörður close to Reykjavik. There is an aluminum smelter in } \\
\text { the area }\end{array}$ \\
\hline
\end{tabular}

\section{Fox sampling}

The samples of liver tissue were collected from arctic. Several animals were hunted during the summer and autumn 2002 (frosen samples). The summary of the samples with background information is presented in table 16

TABLE 18 The samples of arctic fox collected in Iceland 2002. Joint Nordic screening project on musk substances. Environment \& Food Agency (UST)

\begin{tabular}{|c|c|c|c|c|c|c|}
\hline Code & $\begin{array}{l}\text { Hunting } \\
\text { date }\end{array}$ & Site (all rural) & Sex & Weigt & Stomach contents & Age guess \\
\hline 7023 & 25.10 .200 & $\begin{array}{l}\text { Miðnesheidi, við } \\
\text { 2Norðurkot }\end{array}$ & male & $5,2 \mathrm{~kg}$ & $\begin{array}{l}80 \% \text { fish \& } 20 \% \\
\text { seabird }\end{array}$ & $>1$ years \\
\hline 7024 & 23.10 .200 & 2 Sandgerdi, við Tjörnina & male & $4,3 \mathrm{~kg}$ & $\begin{array}{l}90 \% \text { sea bird \& } 10 \% \\
\text { mouse }\end{array}$ & $>1$ years \\
\hline 7033 & 18.5 .200 & $\begin{array}{l}\text { Kirkjuhvammshreppur, } \\
\text { 2Illugastadaland }\end{array}$ & female & $3,5 \mathrm{~kg}$ & Small bird & $>1$ years \\
\hline 7035 & 16.6 .200 & $\begin{array}{l}\text { Dverárhreppur, N- } \\
\text { 2Krossanesfell }\end{array}$ & female & $3,5 \mathrm{~kg}$ & $\begin{array}{l}\text { Meat, egg \& small } \\
\text { bird }\end{array}$ & $>1$ years \\
\hline 7038 & 22.6 .200 & $\begin{array}{l}\text { Strandahreppur, } \\
\text { 2trjótdalur }\end{array}$ & male & $4,0 \mathrm{~kg}$ & $\begin{array}{l}\text { Large bird, small bird, } \\
\text { sea weed }\end{array}$ & $>1$ years \\
\hline 7039 & 22.6 .200 & $\begin{array}{l}\text { Strandahreppur, } \\
\text { 2Grjótdalur }\end{array}$ & female & $3,7 \mathrm{~kg}$ & Small bird & $>1$ years \\
\hline
\end{tabular}

Fox teeth were sent to NERI in January for detailed age determination.

TABLE 19 Coordinates of rainwater sampling sites in Iceland

\begin{tabular}{|c|c|}
\hline Site & Coordinates \\
\hline Miðnesheidi (7023) & Lambert 2 Parallels Northing:394813 Easting: 319663 \\
\hline Sandgerdi (7024) & $64^{\circ} 2,292^{\prime} \mathrm{N},-22^{\circ} 42,173^{\prime} \mathrm{V}$ (ISN93 - WGS84) \\
\hline $\begin{array}{l}\text { Húnavatnssýsla, Illugastadaland } \\
\text { (7033) }\end{array}$ & Lambert 2 Parallels Northing:569138 Easting: 413209 \\
\hline 16.6 .2002 & \\
\hline Árnessýsla, Grjótdalur (7038) & Lambert 2 Parallels Northing: 367577 Easting: 435527 \\
\hline Árnessýsla, Grjótdalur (7039) & Lambert 2 Parallels Northing: 367577 Easting: 435527 \\
\hline
\end{tabular}




\section{Norway}

The Norwegian samples were (if no other is stated) collected and prepared according to the manual given by NERI (see appendix 1). The location of the Norwegian sampling sites and geographical coordinates and classification of the sites in relation to pollution can be found in table 20 Number of specimen collected from different sites is summarized in table 21

TABLE 20 Coordinates of the sampling sites in Norway year 2002. Joint Nordic screening project on musk substances.

\begin{tabular}{|l|l|l|l|}
\hline Sampling site & North & East & Site classification \\
\hline Gressholmen (Oslofiord, Station 30 A). & 59.88 & 10.71 & Urban (marine) \\
\hline Gåsøya (Oslofiord, Station 304) & 59.85 & 10.59 & Suburban (marine) \\
\hline Ramton (Oslofiord, Station 307) & 59.74 & 10.52 & Suburban (marine) \\
\hline Færder (Oslofiord, Station 36A) & 59.03 & 10.53 & Pristine (marine) \\
\hline $\begin{array}{l}\text { Strømmen } \\
\text { (AS Sentral-renseanlegget RA-2) }\end{array}$ & 59.95 & 11.04 & Suburban \\
\hline $\begin{array}{l}\text { Oslo } \\
\text { (Bekkelaget Sewage Treatment Plant) }\end{array}$ & 59.88 & 10.77 & Urban \\
\hline $\begin{array}{l}\text { Vinterbro } \\
\text { (Nordre Follo Sewage Treatment Plant) }\end{array}$ & 59.75 & 10.78 & Suburban \\
\hline $\begin{array}{l}\text { Rygge } \\
\text { (Fuglevik Sewage Treatment Plant) }\end{array}$ & 59.38 & 10.66 & Urban to suburban \\
\hline Lista (Rain water) & 58.10 & 06.57 & Rural (coastal) \\
\hline Hurdal (Rain water) & 60.37 & 11.07 & Rural (forest) \\
\hline Bærum (Fox) & 59.90 & 10.58 & Suburban \\
\hline Oslo (Fox) & 59.93 & 10.68 & Suburban \\
\hline Spitsbergen (Fox) & 78.30 & 17 & Pristine (arctic) \\
\hline
\end{tabular}

TABLE 21 Summary of the samples collected at the sampling sites in Norway 2002. Joint Nordic screening project on musk substances.

\begin{tabular}{|l|c|l|l|l|}
\hline Specimen & $\begin{array}{c}\text { Total number of } \\
\text { samples }\end{array}$ & $\begin{array}{c}\text { Urban + suburban } \\
\text { sites (n) }\end{array}$ & $\begin{array}{c}\text { Forest+rural } \\
\text { sites (n) }\end{array}$ & $\begin{array}{c}\text { Pristine sites } \\
\text { (n) }\end{array}$ \\
\hline Sludge & 5 & $\begin{array}{l}\text { Strømmen (1) } \\
\text { Oslo (2) } \\
\text { Vinterbro (1) } \\
\text { Rygge (1) }\end{array}$ & & \\
\hline Rain water & 4 & Frrder (1) & \\
\hline Blue mussel & 4 & $\begin{array}{l}\text { Gresholmen (1) } \\
\text { Gåsøya (1) } \\
\text { Ramton (1) }\end{array}$ & $\begin{array}{l}\text { Lista (3) } \\
\text { Hurdal (1) }\end{array}$ & \\
\hline Red fox & 2 & $\begin{array}{l}\text { Oslo (1) } \\
\text { Bærum (1) }\end{array}$ & & Spitsbergen (1) \\
\hline Arctic fox & 1 & & & \\
\hline
\end{tabular}




\section{Sludge sampling}

The samples were collected by Svein Stene Johansen, Norwegian Institute of Water Research (NIVA). All single samples are summarized in table 22

TABLE 22 Sludge samples collected by Norway 200 for joint Nordic screening project on musk substances. Sampling by Svein Stene Johansen, Norwegian Institute of Water Research (NIVA).

\begin{tabular}{|c|c|c|c|}
\hline Sample & Site /sewage plant & $\begin{array}{l}\text { Person } \\
\text { equivalent } \\
\text { (peq) }\end{array}$ & Classification \\
\hline Sample 1. & $\begin{array}{l}\text { Strømmen, AS Sentralrenseanlegget RA-2 } \\
(27.08 .02 \text { at } 08.30 \mathrm{hrs} .) \\
\text { Chemical treatment }\end{array}$ & 103.000 & $\begin{array}{l}\text { Suburban (percentage } \\
\text { industrial waste water not } \\
\text { known) }\end{array}$ \\
\hline Sample 2. & $\begin{array}{l}\text { Oslo, Bekkelaget Sewage Treatment Plant } \\
(27.08 .02 \text { at } 09.45 \text { hrs.) } \\
\text { Chemical and biological treatment with } \\
\text { nitrogen removal }\end{array}$ & 280.000 & $\begin{array}{l}\text { Urban (with ca. } 30 \% \\
\text { industrial waste water) }\end{array}$ \\
\hline Sample 3. & $\begin{array}{l}\text { Oslo, Bekkelaget Sewage Treatment Plant } \\
(27.08 .02 \text { at } 09.45 \text { hrs.) } \\
\text { Same as above }\end{array}$ & $\begin{array}{l}\text { Same as } \\
\text { above }\end{array}$ & Same as above \\
\hline Sample 4. & $\begin{array}{l}\text { Vinterbro, Nordre Follo Sewage Treatment } \\
\text { Plant ( } 27.08 .02 \text { at } 11.15 \text { hrs.) } \\
\text { Chemical and biological treatment }\end{array}$ & 70.000 & $\begin{array}{l}\text { Suburban (with an } \\
\text { estimation of } 30 \% \\
\text { industrial waste water, } \\
\text { including a factory that } \\
\text { produces cosmetics etc.) }\end{array}$ \\
\hline Sample 5. & $\begin{array}{l}\text { Rygge, Fuglevik Sewage Treatment Plant } \\
(27.08 .02 \text { at } 12.15 \text { hrs.) } \\
\text { Chemical treatment }\end{array}$ & 50.000 & Urban to suburban \\
\hline
\end{tabular}

\section{Rain water sampling}

Sampling of rain water was lead by Martin Schlabach, Norwegian Institute of Air Research (NILU). Three samples of rain water were collected at Lista and two were collected in Hurdal. All samples are listed in table 23

Description of the sampling equipment: Bulk sampling, glass funnel with $285 \mathrm{~mm}$ diameter directly connected to a 2 liter glass bottle. Funnel is permanently open (a wet only system is not used). Height above ground level: $2 \mathrm{~m}$. 
TABLE 23 Samples of rainwater collected in Norway 2002. Joint Nordic screening project on musk substances. Sampling led by Martin Schlabach, Norwegian Institute of Air Research (NILU).

\begin{tabular}{|c|c|c|c|c|}
\hline Sample & Site & Sampling period & Volume (L) & Remarks \\
\hline $\begin{array}{l}\text { Sample } \\
\text { Lista } 1\end{array}$ & Lista & $07.10 .02-24.10 .02$ & 2 liter & $\begin{array}{l}\text { Rural. Immedialte } \\
\text { surroundings : } \\
\text { Grass } 95 \% \text {, forest } 5 \% \text {. } \\
\text { Ca. } 1 \mathrm{~km} \text { from Elkem } \\
\text { (industy) } 13 \mathrm{~m} \text { a.s.l. } \\
\end{array}$ \\
\hline $\begin{array}{l}\text { Sample } \\
\text { Lista } 2\end{array}$ & Lista & $25.10 .02-06.11 .02$ & 2 liter & Same as above \\
\hline $\begin{array}{l}\text { Sample } \\
\text { Lista } 3\end{array}$ & Lista & $06.11 .02-07.11 .02$ & 2 liter & Same as above \\
\hline $\begin{array}{l}\text { Sample } \\
\text { Hurdal } 1\end{array}$ & Hurdal & $16.10 .02-28.10 .02$ & & $\begin{array}{l}\text { Immediate surroundings: } \\
\text { Forest with clearing } \\
\left(\text { about } 3000 \mathrm{~m}^{2}\right) \text {. About } \\
700 \mathrm{~m} \text { from nearest farm } \\
\text { and road. } 300 \mathrm{~m} \text { a.s.l. }\end{array}$ \\
\hline $\begin{array}{l}\text { Sample } \\
\text { Hurdal } 2\end{array}$ & Hurdal & $28.10 .02-01.11 .02$ & 2 liter & Same as above \\
\hline
\end{tabular}

\section{Blue mussel sampling}

Sampling of mussels was performed by Norwegian Institute of Water Research (NIVA) The living mussels were rinsed according to the manual and depurated for 12 hours in water form the sampling site for 12 hours in a refrigerated room. All samples are summarized in table 24

Table 24 Blue mussel samples collected in different parts of the Oslofiord. Joint Nordic screening project on musk substances. Sampling led by Norman Greene, Norwegian institute of Water Research (NIVA).

\begin{tabular}{|l|l|l|l|l|}
\hline Sample & Date & $\begin{array}{l}\text { Site } \\
\text { (name, depth in m) }\end{array}$ & $\begin{array}{l}\text { Size class (mm); } \\
\text { mean }\end{array}$ & Remarks \\
\hline $\begin{array}{l}\text { Sample } \\
\text { St. 30A }\end{array}$ & 01.10 .02 & $\begin{array}{l}\text { Oslofiord, Gressholmen } \\
\text { (Station 30 A) } \\
0,5-1 \mathrm{~m} .\end{array}$ & $\begin{array}{l}45,2 \pm 2,6 \\
(40 \text { individuals })\end{array}$ & $\begin{array}{l}\text { Close to Oslo harbour in } \\
\text { the Oslofiord, 16 km from } \\
\text { Oslo's main sewage } \\
\text { treatment plant at } \\
\text { Slemmestad }\end{array}$ \\
\hline $\begin{array}{l}\text { Sample } \\
\text { St 304 }\end{array}$ & 01.10 .02 & $\begin{array}{l}\text { Oslofiord, Gåsøya } \\
\text { (Station 304) } \\
1 \text { m. }\end{array}$ & $\begin{array}{l}45,3 \pm 2,8 \\
(40 \text { individuals })\end{array}$ & $\begin{array}{l}\text { Inner part of Oslofiord, } 9 \\
\text { km from Oslo's main } \\
\text { sewage treatment plant at } \\
\text { Slemmestad }\end{array}$ \\
\hline $\begin{array}{l}\text { Sample } \\
\text { St 307 }\end{array}$ & 01.10 .02 & $\begin{array}{l}\text { Oslofiord, Ramton } \\
\text { (Station 307) } \\
0,5 \mathrm{~m}\end{array}$ & $\begin{array}{l}44,5 \pm 2,4 \\
(40 \text { individuals })\end{array}$ & $\begin{array}{l}\text { Inner part of Oslofjord, } \\
\text { 4 km from Oslo's main } \\
\text { sewage treatment plant at } \\
\text { Slemmestad }\end{array}$ \\
\hline $\begin{array}{l}\text { Sample } \\
\text { St 36A }\end{array}$ & 11.10 .02 & $\begin{array}{l}\text { Oslofiord, Færder } \\
\text { (Station 36A) } \\
0,5-1 \mathrm{~m} .\end{array}$ & $\begin{array}{l}47,6 \pm 5,7 \\
(30 \text { individuals })\end{array}$ & $\begin{array}{l}\text { At the outmoust part } \\
\text { Oslofiord. Can probably be } \\
\text { seen as a reference } \\
\text { condition station for } \\
\text { northern Skagerrak and } \\
\text { inner part of Oslofjord. }\end{array}$ \\
\hline
\end{tabular}




\section{Fox samples}

Only two samples of red fox (Vulpes vulpes) could be collected from the Oslo area during the autumn of 2002. In addition we have delivered one sample of Arctic fox (Alopex lagopus) from Spitsbergen. The arctic foxes on Svalbard feed on both the terrestrial and marine food web. The samples of liver tissue from red fox were collected by Anders Gimse (Oslo municipality) and Paul Berger Hansen (Bærum municipality) and prepared and described by Kjell Handeland, National Veterinary Institute. The samples of liver tissue from arctic fox were collected, prepared and described by Eva Fuglei, Norwegian Polar Institute.

The summary of the samples with background information is presented in table 25

TABLE 25 The samples of red fox and arctic fox collected in Norway 2002. Joint Nordic screening project on musk substances.Sampled by Eva Fuglei (Norwegian Polar Institute), Anders Gimse (Oslo municipality) and Paul Berger Hansen (Bærum municipality).

\begin{tabular}{|l|l|l|l|l|l|}
\hline Sample & Animal & Sex & Hunting date & $\begin{array}{l}\text { Age } \\
\text { (year) }\end{array}$ & Site and classification \\
\hline $\begin{array}{l}\text { Sample, } \\
\text { Spitsbergen }\end{array}$ & Arctic fox & male & February 2002 & 8 & $\begin{array}{l}\text { Spitsbergen, pristine (arctic) } \\
32 \text { km from Longyearbyen }\end{array}$ \\
\hline Sample, Oslo & Red fox & male & 05.11 .2002 & $\begin{array}{l}\text { Young } \\
\text { animal }\end{array}$ & $\begin{array}{l}\text { Ullern in Oslo municipality, } \\
\text { suburban }\end{array}$ \\
\hline Sample, Bærum & Red fox & female & 14.10 .2002 & Adult & $\begin{array}{l}\text { Høvik in Bærum municipality, } \\
\text { suburban }\end{array}$ \\
\hline
\end{tabular}




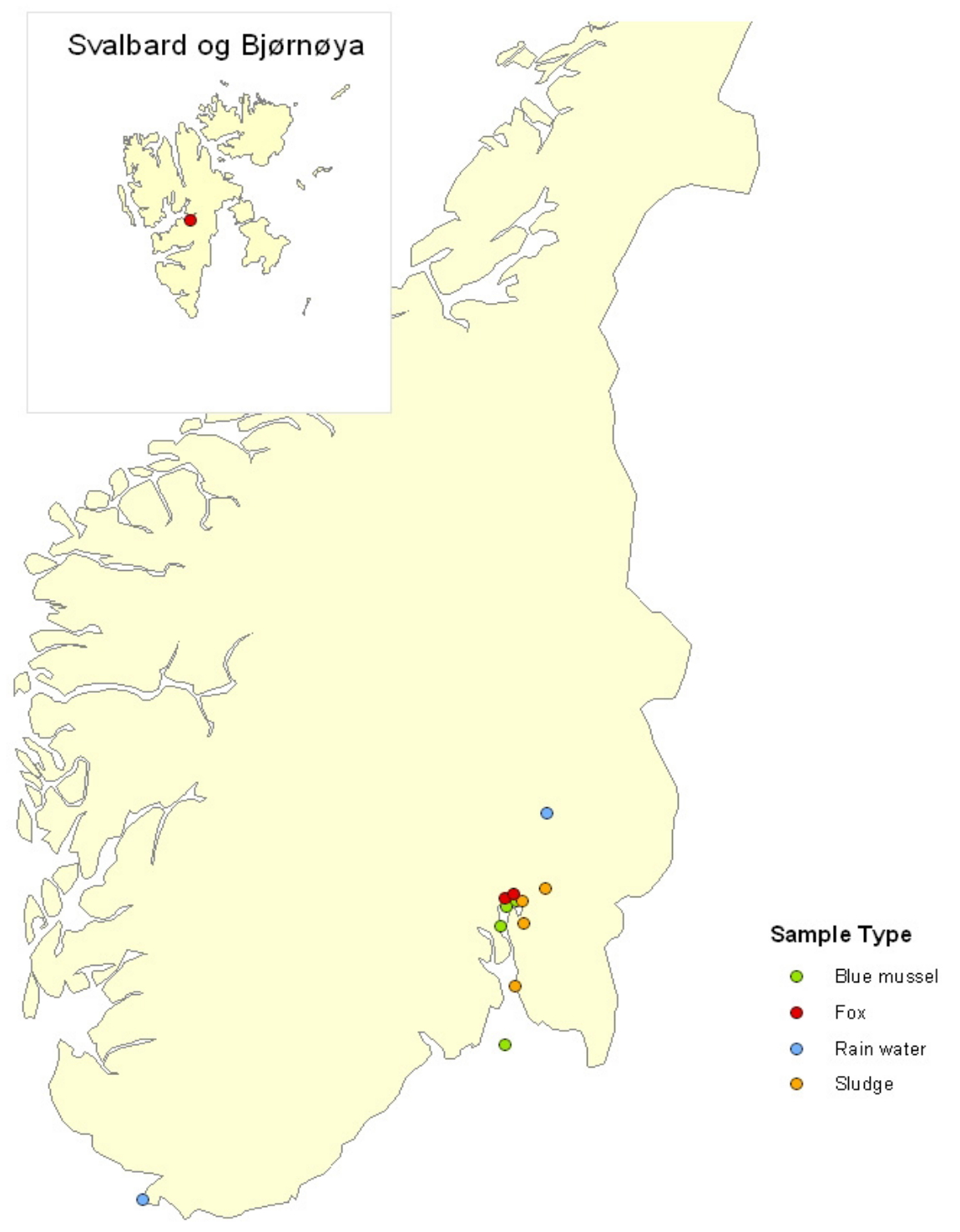

Figure 5 Sample sites in Norway. 


\section{Sweden}

\section{Sludge sampling}

Sampling was performed according to the method description of NERI Appendix 1.

TABLE 26 Sludge samples taken

\begin{tabular}{|l|c|c|l|c|}
\hline \multicolumn{1}{|c|}{ Site /sewage plant } & $\begin{array}{c}\text { Person } \\
\text { equivalent } \\
\text { (peq) }\end{array}$ & $\begin{array}{c}\text { Sampling } \\
\text { date }\end{array}$ & \multicolumn{1}{|c|}{ Classification } & Coordinate \\
\hline Vimmerby & $>10000$ & $2002-11-18$ & municipal & $57.67^{\circ} \mathrm{N}, 15.85^{\circ} \mathrm{E}$ \\
\hline Stenungsund & $>20000$ & $2002-11-20$ & Industrial+municipal & $58.07^{\circ} \mathrm{N}, 11.82^{\circ} \mathrm{E}$ \\
\hline Stockholm:/Henriksdal & $>400000$ & $2002-11-18$ & Municipal (big city) & $59.32^{\circ} \mathrm{N}, 18.07^{\circ} \mathrm{E}$ \\
\hline
\end{tabular}

Notes: There are possible emitters of musk substances in the area, either industrial or households No known musk sources; in Stenungsund, however, large chemical industry nearby, e.g. vinyl flooring industry Weather condition during the sampling and the week before:

Vimmerby: overcast, occasional rain, +5 degrees $\mathrm{C}$

Stenungsund: Overcast, some rain, a few plus degrees Celsius

Bromma: Occasional rain, a few +-degrees Celsius

\section{Rain water sampling}

The sampling apparatus requested by NERI in their manual couldn't be used and was not available. The rain samples were collected using a glass funnel with a diameter of $20 \mathrm{~cm}$ connected to a glass bottle. The sampler was wrapped in aluminum foil during the sampling. Two samplers were used in parallel at each sampling site.

TABLE 27: Rain water samples taken

\begin{tabular}{|l|l|l|l|l|}
\hline Station & Classification & Sampling date & Volume & Coordinate \\
\hline Pallas & $\begin{array}{l}\text { Background station, forest } \\
\text { area }\end{array}$ & & & $75.65^{\circ} \mathrm{N}, 18.47^{\circ} \mathrm{E}$ \\
\hline Råö & $\begin{array}{l}\text { Background station, } \\
\text { Forest area }\end{array}$ & $\begin{array}{l}2002-09-09- \\
2002-10-16\end{array}$ & & $63.70^{\circ} \mathrm{N} 12.66^{\circ} \mathrm{E}$ \\
\hline
\end{tabular}

Notes: Weather conditions during the sampling period 


\section{Blue mussel sampling}

Samplings of Blue mussels were carried out according to Nordic Environmental Specimen Banking - methods in use in ESB. Manual for the Nordic countries.

(TemaNord 1995:543).

Blue mussels were collected by hand from the inter tidal zone. It is recommended that sampling should be done as near to the same depth and exposure (in respect to sunlight and wave action) as possible. Late autumn is recommended as sampling time because mussels are in a less unstable physiological state. The blue mussels were pooled in size groups according to shell length, each group consisting of 20 individuals.

TABLE 28. Blue mussel samples collected.

\begin{tabular}{|l|l|l|l|l|}
\hline Sample code & Date & Site* $($ depth, m) & $\begin{array}{l}\text { Size class }(\mathbf{m m}) \\
(\mathbf{m e a n} \pm \text { SD) }\end{array}$ & Coordinate \\
\hline $\begin{array}{l}\text { Kolerakyrkogården } \\
\text { Bohus-Malmön }\end{array}$ & $2002-09-01$ & $1-1.5 \mathrm{~m}$ & $60+-10$ & $\begin{array}{l}58.21^{\circ} \mathrm{N} \\
11.19^{\circ} \mathrm{E}\end{array}$ \\
\hline
\end{tabular}

Remarks: Possible industrial source of musk substances in the area. 


\section{BELGIUM \& LUXEMBOURG}

Jean de Lannoy

Avenue du Roi, 202, 1190 Brussels

Tel +32 (0)2 $5385169 \mathrm{Fax}+32$ (0)25380841

jean.de.lannoy@euronet.be

\section{CANADA}

Renouf Publishing Company Ltd

5369 Canotek Road, Ottawa, Ontario K1J 9J3

Tel + 1 (613) $7452665 \mathrm{Fax}+1$ (613) 7457660

order.dept@renoufbooks.com

www.renoufbooks.com

\section{CHINA}

CNPIEC

Europe Division 16 Gongti East Road, P.O. Box 88, Beijing

Tel +86105066 688-8 Fax +86105063101

\section{DENMARK}

Svensk-Norsk Bogimport A/S

Esplanaden 8 B, 1263 København $\mathrm{K}$

Tel +4533142666 Fax +4533143588

snb@bog.dk

www.snbog.dk

\section{ESTLAND}

Astro Raamatud AS

Pärnu mnt 142, 11317 Tallinn

Tel +372 6548485 Fax +372 6548475

book@astro.ee

\section{FAROE ISLANDS}

H.N. Jacobsens Bókahandil

Postboks 55, 110 Tórshavn

Tel +298311036 Fax +2983178 73

hnj-bokh@post.olivant.fo

\section{FINLAND}

Akademiska Bokhandeln

PB 128, Centralgatan 1, 00101 Helsingfors

Tel +358 912141

akatilaus@akateeminen.com

www.akateeminen.com

\section{FRANCE}

Librairie LAVOISIER

14, rue de Provigny, 94236 Cachan Cedex

Tel +33 (1) $47406700 \mathrm{Fax}+33$ (1) 47406702

group@lavoisier.fr

www.lavoisier.fr

\section{GERMANY}

UNO-Verlag GmbH

Am Hofgarten 10, 53113 Bonn

Tel +49 (0)228949020 Fax +49 (0)2289490222

info@uno-verlag.de

www.uno-verlag.de

\section{HUNGARY}

Euro Info Service

PO Box 1039, 1245 Budapest

Tel +36 (1) $3292487 \mathrm{Fax}+36$ (1) 3492053

euroinfo@euroinfo.hu

\section{ICELAND}

Mál og Menning
Laugavegi 18, 101 Reykjavik

Tel +354 (9)5152500 Fax +354 (9)5152505

verslun@mm.is

\section{LATVIA}

Jana Rozes Gramàtnica

Kr. Barona iela 5, 1011 Riga

Tel +371 (0)2 284288 Fax +371 7370922

\section{LITHUANIA}

Penki Kontinentai

A. Stulginskio 5, 2001 Vilnius

Tel +370 (5) $2664540 \mathrm{Fax}+370$ (5) 2664565

books@5ci.lt

www.books.It

\section{NORWAY}

Akademika A/S

Postboks 84 Blindern, 0314 Oslo

Tel +4722853030 Fax +4722 853080

bloken@sio.uio.no

www.akademika.no

\section{ROMANIA}

Euromedia s.r.l.

Str Dionisie Lupu nr 65, 70184 Bucuresti

Tel + 4016140664 Fax + 4013129646

\section{SWEDEN}

Fritzes

Kundservice, 10647 Stockholm

Tel +46 (0)8 $6909190 \mathrm{Fax}+46$ (0)8 6909191

order.fritzes@nj.se

www.fritzes.se

\section{THE NETHERLANDS}

De Lindeboom Internationale Publicaties b.v.

M.A. de Ruyterstraat 20 A, NL-7482 BZ Haaksbergen

Tel +31 (0)53 5740004, Fax +31 (0)535729296

books@delindeboom.com

www.delindeboom.com

\section{UNITED KINGDOM}

The Stationery Office

P.O. Box 276, London SW8 5DT

$\mathrm{Tel}+448706005522 \mathrm{Fax}+448706005533$

customer.services@tso.co.uk

www.tso.co.uk/bookshop

\section{USA}

Bernan

4611-F Assembly Drive, Lanham MD 20706-4391

Tel +1 (301) $4597666 \mathrm{Fax}+1$ (301) 4590056

query@bernan.com

www.bernan.com

\section{ÅLAND}

Lisco bok- och pappershandel

Skarpansvägen 25, Box 8, 22101 Mariehamn Tel +358 (0)1817177 Fax +358 (0)1819771 info@lisco.fi 